\title{
DEMENTIA NARRATIVES OF EMERGENCY DEPARTMENT NURSES
}

By

Negin Pearl Shalchi, BScN, 2008

Ryerson University, Toronto, Ontario

\author{
A thesis \\ presented to Ryerson University \\ in partial fulfillment of the \\ requirements for the degree of \\ Master of Nursing \\ in the Program of \\ Nursing
}

Toronto, Ontario, Canada, 2014

(C)Negin Pearl Shalchi, 2014 


\section{AUTHOR'S DECLARATION}

I hereby declare that I am the sole author of this thesis. This is a true copy of the thesis, including any required final revisions, as accepted by my examiners.

I authorize Ryerson University to lend this thesis to other institutions or individuals for the purpose of scholarly research.

I further authorize Ryerson University to reproduce this thesis by photocopying or by other means, in total or in part, at the request of other institutions or individuals for the purpose of scholarly research.

I understand that my thesis may be made electronically available to the public 


\title{
DEMENTIA NARRATIVES OF EMERGENCY DEPARTMENT NURSES
}

\author{
ABSTRACT \\ Negin Pearl Shalchi \\ Master of Nursing Program \\ Ryerson University, Toronto, 2014
}

The Emergency Department is often the main portal of entry for acutely ill elderly patients with dementia requiring healthcare. Emergency nurses assume responsibility for managing both critical illnesses and Behavioural and Psychological Symptoms of Dementia. Using narrative methodology and photographic images provided by participants, two emergency department nurses were interviewed to understand their experiences caring for patients with dementia. A Critical Social Theory lens facilitated an examination of how dominant discourse about work environment, power relations, and the healthcare system shape their stories of dementia. Findings indicated that nurses’ experiences are shaped by frustrations, threaten personcentred care, and are associated with limited time and knowledge and competing work demands when caring for patients with dementia. Study recommendations include the need for policy that supports culture change in the emergency department, investment in professional development of emergency department nurses, and infrastructure supports such as Geriatric Emergency Management nurses and nurse’s aides. 


\section{ACKNOWLEDGEMENTS}

This thesis is the result of much hard-work and dedication from a number of extraordinary and dedicated people that I would like to take the time to thank.

My heartfelt appreciation goes to Dr. Lori-Schindel Martin, my thesis supervisor, who dedicated countless hours to the progress of this thesis from start to finish. She saw my potential throughout this journey and always encouraged me to push myself just a bit more to come out of my comfort zone. For this I am deeply grateful as it allowed me to learn and grow as a person and as a researcher. I am forever grateful for all your patience, dedication, knowledge and wisdom you have imparted on me.

I would like to express my sincere gratitude to my thesis committee members, Dr. Jennifer Lapum, for all of her creative advice and ongoing academic support and mentorship, Dr. Sharon Kaasalainen and Leslie Gillies, for providing their unique expertise in the development of this thesis. Many thanks go out to each of you for all of your suggestions, time, effort and assistance in contributing to the successful completion of this thesis.

I would like to acknowledge and thank the department of Graduate Studies at Ryerson University, Ontario Graduate Scholarship, North York General Hospital-Frank Gerstein, and the Alzheimer's Society of Hamilton/Halton for their financial support of this research project.

A special thanks to the participants of this study for sharing their time and stories and making this an enjoyable learning experience, without your commitment, this project would not have been possible.

Finally, I would like to thank my friends and colleagues for their willingness to provide assistance when I stumbled across complex technical issues and for your continuous help in editing and proof reading my work. 


\section{DEDICATION}

This thesis is dedicated to my loving and patient mother, Mitra, and to my sisters, Nahal and Lily, who have been a great source of motivation and support since the beginning of my studies. Thank you for being my best cheerleaders and allowing me to turn every room in our home into my office. 


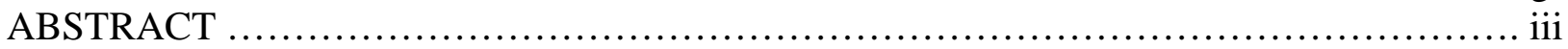

ACKNOWLEDGMENTS ...................................................................

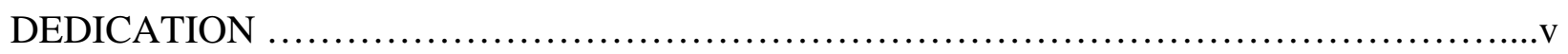

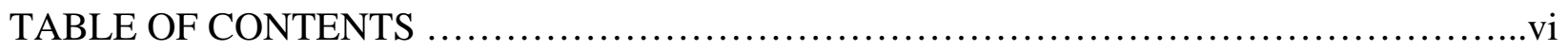

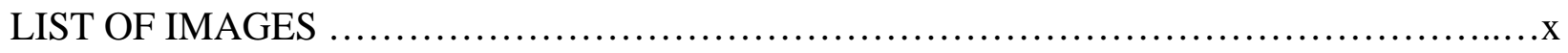

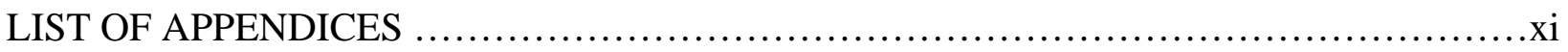

CHAPTER 1: PROLOGUE

THE EMERGENCY DEPARTMENT: MY NOVICE

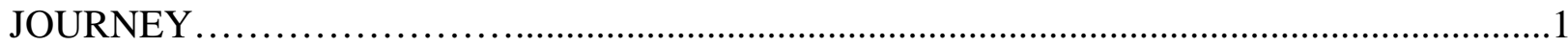

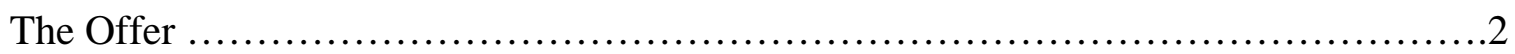

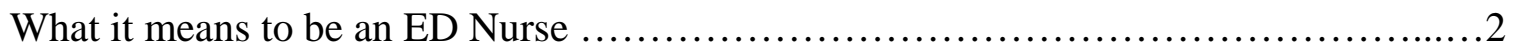

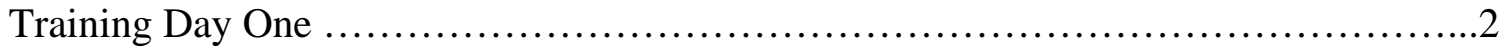

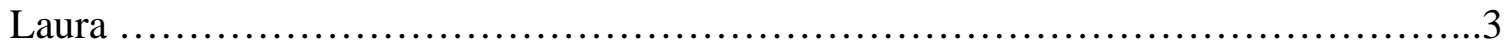

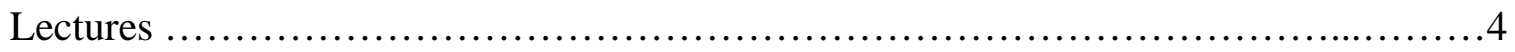

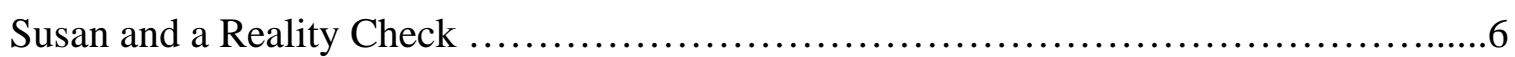

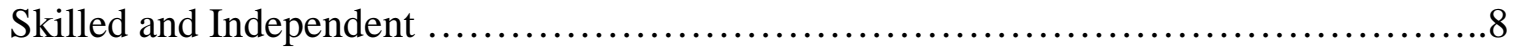

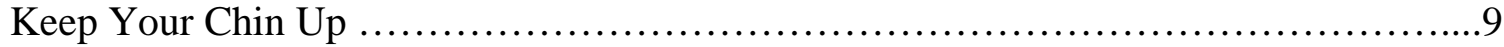

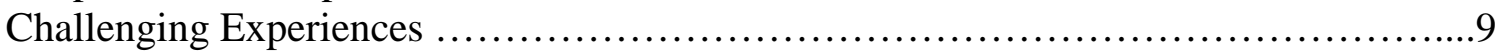

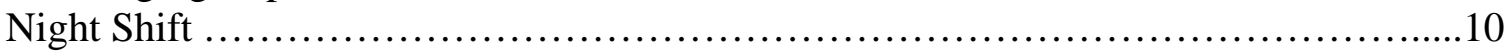

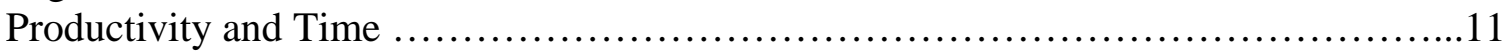

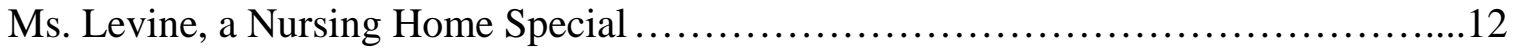

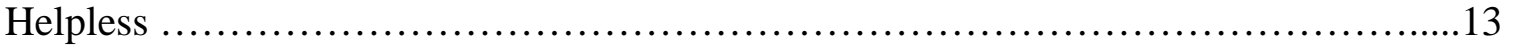

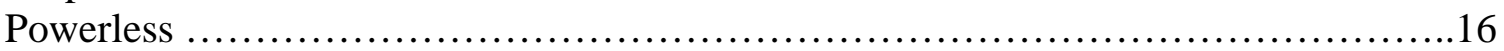

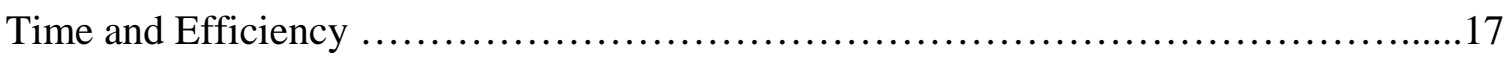

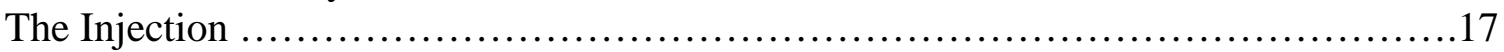

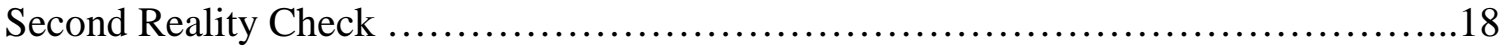

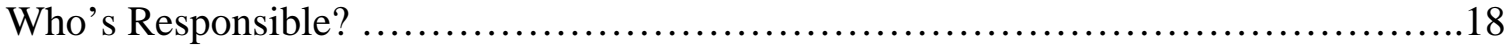

CHAPTER 2: DEMENTIA IN THE EMERGENCY ROOM: A SILENT CRISIS

BACKGROUND, LITERATURE REVIEW \& SYNTHESIS, RESEARCH SIGNIFICANCE, STATEMENT OF STUDY PURPOSE,

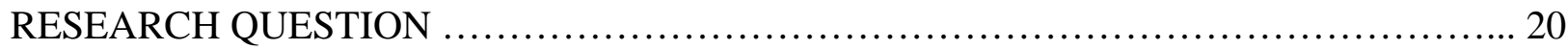

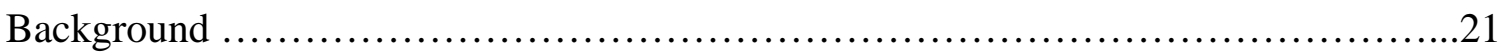

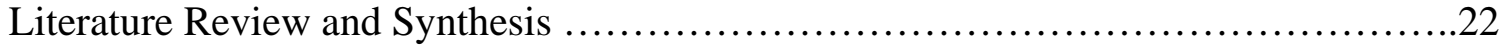

Significance of Dementia Care in the ED ..................................24

Challenging and Responsive Behaviours in Dementia ........................25 
Culture Change and Dementia Care …....................................28

Person-Centred Behavioural Management .................................... 29

The Influence of Environment on Dementia Care ............................... 33

Staff Development in Dementia Care ....................................... 35

Significance of Research Project............................................... 36

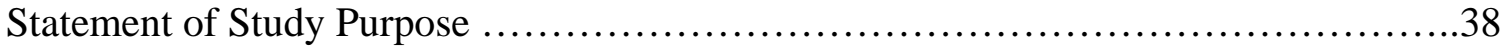

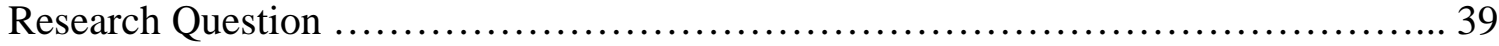

CHAPTER 3: THEORETICAL LENS, METHODOLOGY, AND DESIGN ..................40

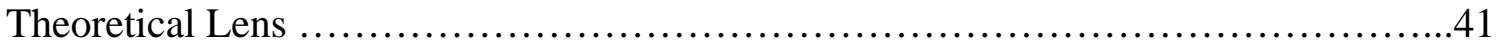

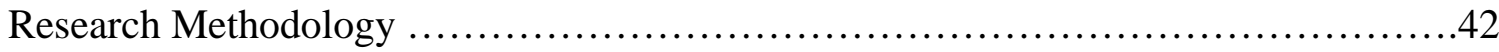

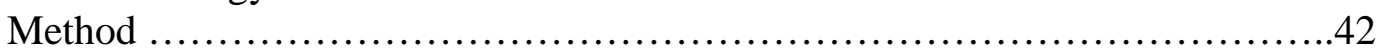

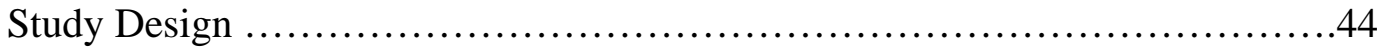

Data Collection .............................................................. 46

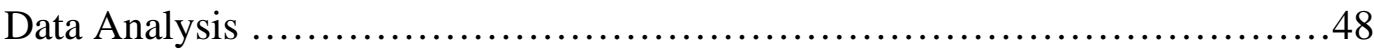

Categorical-content analysis ......................................49

Categorical-form analysis ......................................50

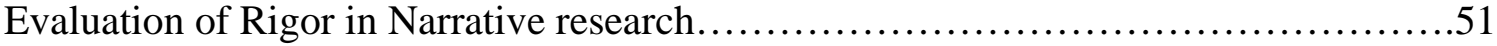

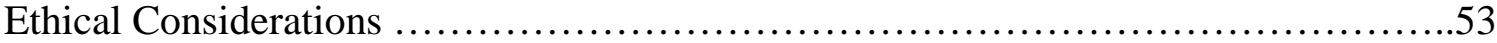

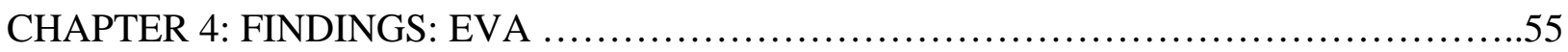

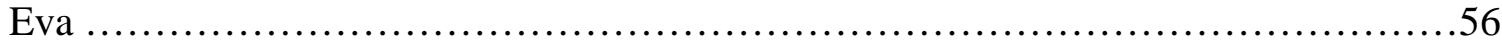

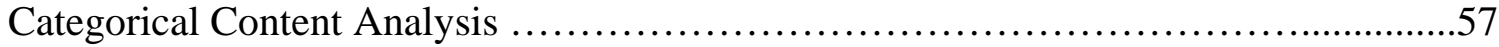

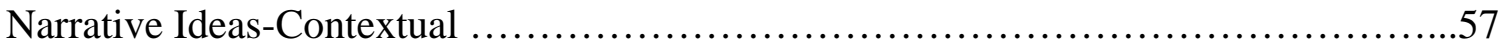

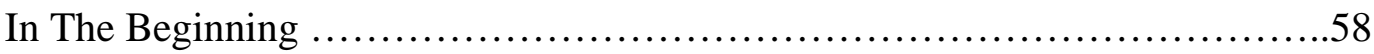

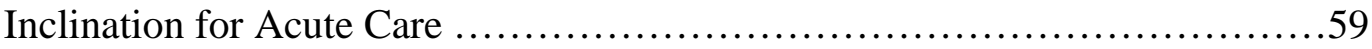

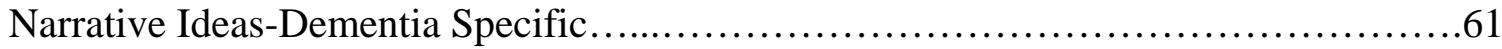

Dementia: Experiential Knowledge ..........................................62

Person-Centred Care: The Impossible .....................................69

Emotional Labour of Work ...................................................73

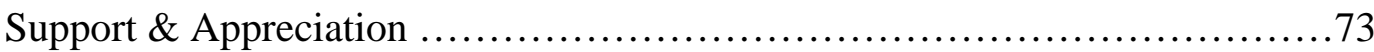

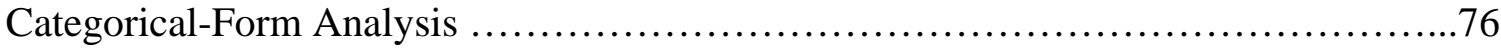

Cognitive Analysis ................................................... 76

Emotional Analysis ........................................................

Linguistics: the said words. ......................................79

Paralinguistics: the unsaid words .....................................80

Formal elements. ....................................................

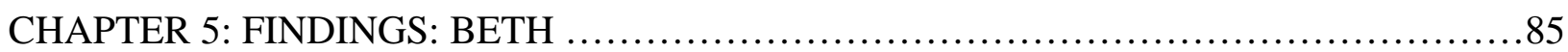

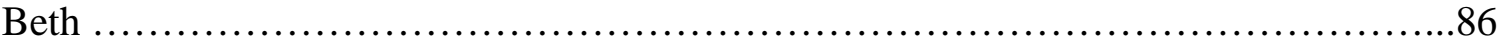

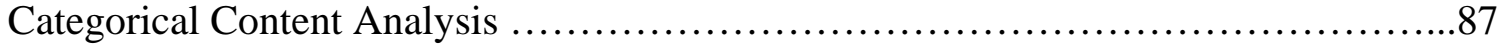

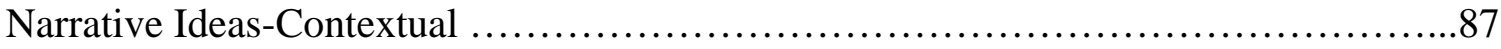




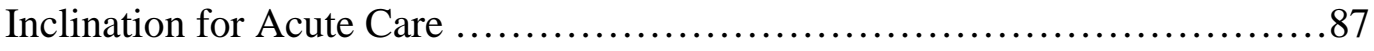

Narrative Ideas-Dementia Specific …............................................99

Dementia: Experiential Knowledge ...........................................99

Person-Centred Care: The Impossible .......................................94

Emotional Labour of Work ................................................99

Support \& Appreciation ...................................................102

Categorical Form Analysis ...................................................... 103

Cognitive Analysis .................................................... 103

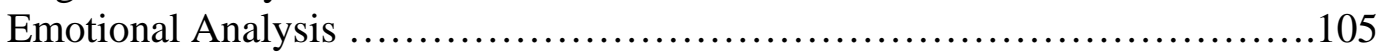

Linguistics: the said words. ....................................105

Paralinguistics: the unsaid words..................................106

Formal elements....................................................

CHAPTER 6: FINDINGS: EVA \& BETH: A COMPARATIVE ANALYSIS ..................110

Eva and Beth: A Comparative Analysis .........................................111

Inclination for Acute Care ..............................................111

Dementia: Experiential Knowledge ..........................................112

Person-Centred Care: The Impossible ....................................114

Emotional Labour of Work ....................................................116

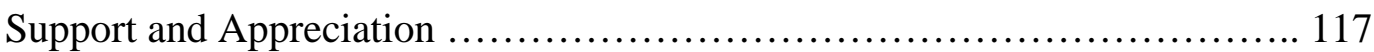

Comparative Analysis Summary ...............................................118

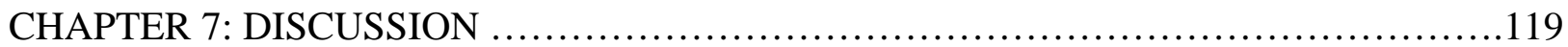

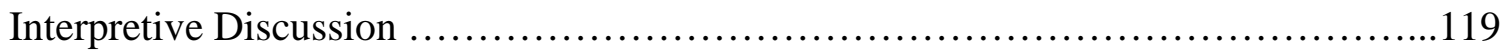

The Glamourized Emergency Department …....................................120

Images of an ED ....................................................... 121

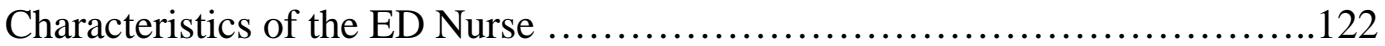

The Exemplary Dementia Care Nurse ....................................123

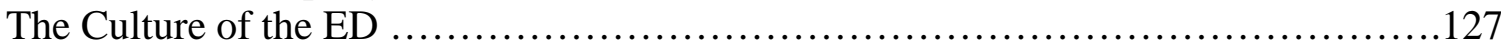

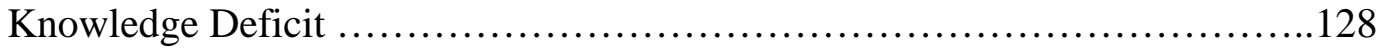

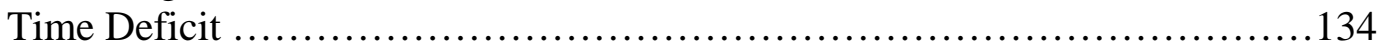

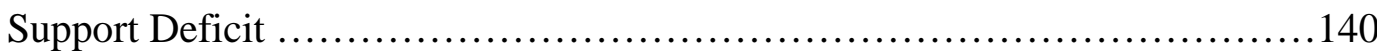

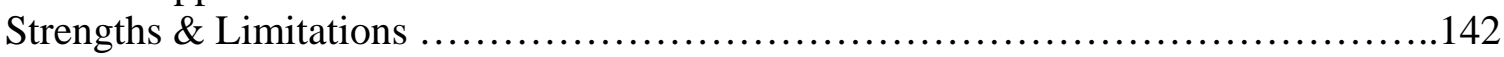

Recommendations: Research, Education, Practice and Policy .........................145

Research ..............................................................

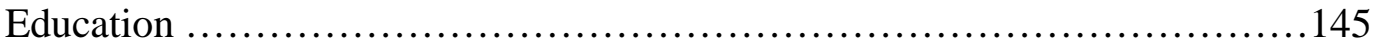

Practice and Policy .....................................................

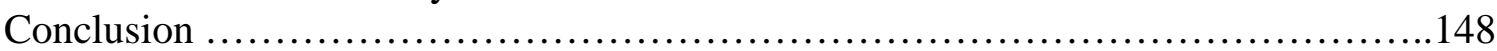

CHAPTER 8: EPILOGUE: MY KNOWLEDGE INFORMED PRACTICE...................150

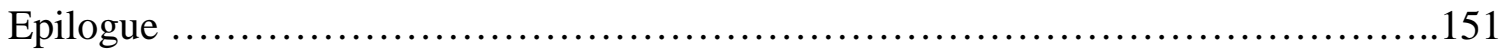

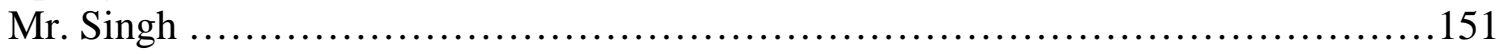

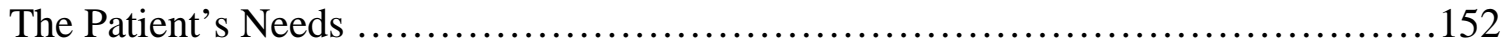

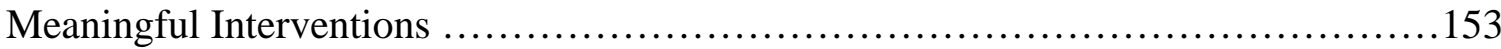

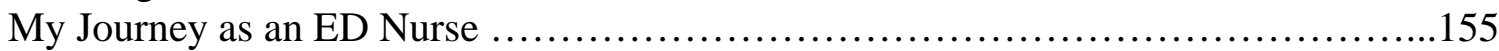




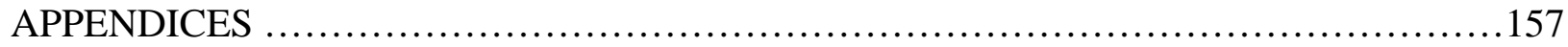

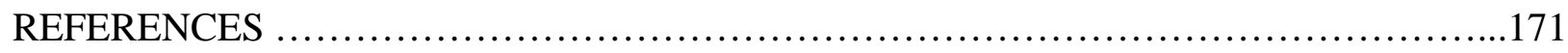

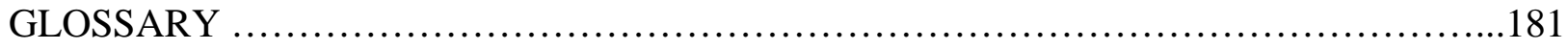




\section{LIST OF IMAGES}

Image 1. Alone \& Helpless ........................................................... 16

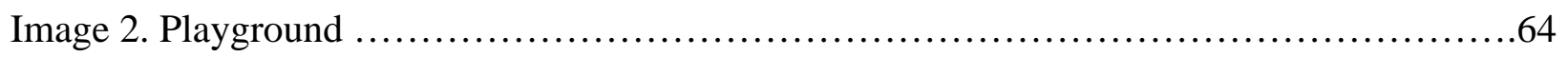

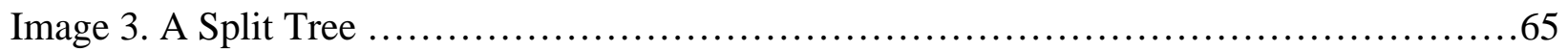

Image 4. A Lonely Path .......................................................... 71

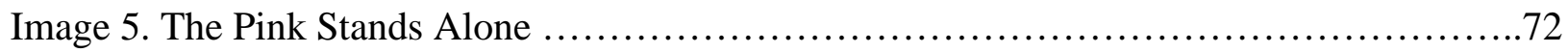

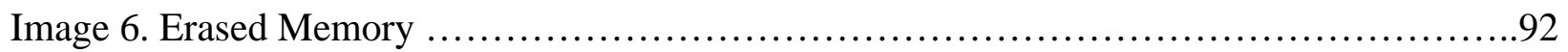

Image 7. Clinical Perception of Dementia ..........................................93

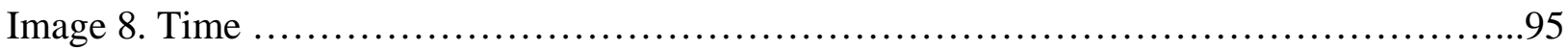

Image 9. Wait Times ...........................................................96

Image 10. Restraints: At Times Necessary ..........................................98

Image 11. Constant Repetition ................................................... 101

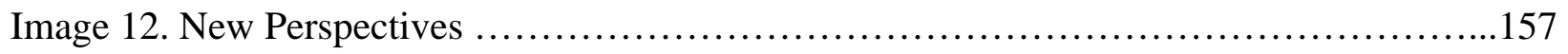




\section{LIST OF APPENDICES}

APPENDIX A: EMERGENCY DEPARTMENT TRENDS ..............................159

APPENDIX B: HEALTH SERVICE USE TRENDS ................................ 160

APPENDIX C: INFORMATION/INVITATION LETTER .............................161

APPENDIX D: RECRUITMENT POSTER .......................................... 162

APPENDIX E: INFORMATION \& CONSENT FORM ................................163

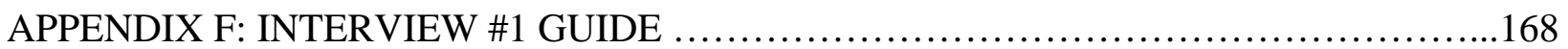

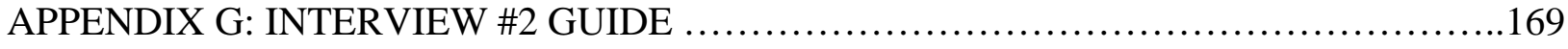

APPENDIX H: PHOTOGRAPHY ACTIVITY INFORMATION SHEET ..................170

APPENDIX I: CAMERA COLLECTION MEETING GUIDE ...........................171 
CHAPTER 1

PROLOGUE

THE EMERGENCY DEPARTMENT: MY NOVICE NURSE JOURNEY 


\section{The Offer}

It is the 20th of April, 2008. Today, one week after graduation from an undergraduate program in nursing, I receive a phone call from the Human Resource Department that an offer of employment as a Registered Nurse (RN) in the Emergency Department (ED) has been extended to me. Considering that I have no prior ED experience, I am in sheer disbelief that they have chosen to employ me. Nursing orientation begins in three weeks, providing me with plenty of time to take pleasure in relaxing rituals prior to beginning a new chapter in my life. The characters I will introduce in the following account of my experiences have been given pseudonyms as a measure to protect their privacy.

\section{What it means to me to be an ED Nurse}

Although I have no previous exposure to the ED, it continues to serve as the most appealing clinical area based on the positive conversations I had with fellow nursing peers who rotated in the ED for their clinical practicums. Their stories were different from what I had experienced on most wards as a nursing student: administering medications, being physically exhausted from changing soiled briefs, cleaning bedpans, and bathing patients every shift, and even multiple times a shift. I've heard from ED nurses in the profession that there is no time for all that "holistic stuff" they teach you in nursing school. In the ED you get to the bottom line; you discover what the patient is there for, treat their problem, and quickly transfer them to the wards or discharge them home.

\section{Training Day One}

It was a brisk Monday morning in May of 2008 and a very important day for me; it was training day one. After a longer than anticipated walk from my car to the front doors of the hospital, I apprehensively walked through the automatic hands-free large revolving glass doors 
into a beautifully crafted front lobby. Inside, the lobby was surrounded by large windows that stretched from floor to ceiling allowing all the daylight to brighten the foyer. There sat several modern blue coloured single person cushioned chairs on either side of the revolving doors where people could take pleasure in the view of the large atrium, beautiful art work, and donor plaques on the drop down walls eloquently centred in the foyer. The sweet aroma of fresh coffee from the lobby Starbucks lingered in the air and in a moment its familiar smell and the symbolic twotailed mermaid logo on the cup instilled comfort within me. A few feet away I sauntered over to the three elevator cars where the "up" button was already illuminated by one of several early morning passengers who were awaiting a ride.

\section{Laura}

My dream became more apparent when I stepped off the elevator and walked about ten feet towards what appeared to be the training room. Outside the room stood about 15 young women, actively engaged in discussion, mostly in their early 20s, all aspiring to be ED nurses, just like me. There was not a single familiar face. Walking towards the door from the other end of the hall was the ED educator, Laura. She was pushing a small grey cart stacked with training manuals. While she slipped the key into the door, Laura smiled and sarcastically announced in a bubbly tone "I'm glad to see that we all made it in this morning." My inner voice questioned the authenticity of Laura's remark; did she expect a much less perfect attendance on subsequent days, or perhaps she anticipated fewer punctual days. Either way, Laura’s comment was disheartening and left me with ambivalent feelings about the all-encompassing and lengthy training program that I had committed myself to. I had to be positive. After a speedy internal monologue I was convinced that I would withstand any challenge. I would impress Laura by arriving on time and with the same enthusiasm that I displayed that day. 
The chatter continued as we simultaneously and with some hesitation made our way into a large well lit room with orange brown laminate flooring. Before us were four round tables accompanied by several office chairs with mesh seating and roller legs and a large suspended ceiling projector used for presentations. The formal elements of this room gave it a sense of productivity, some place where important decisions were made, like a boardroom; it was somewhat cold and intimidating. Working groups were quickly established; three tables of four and one table of three people were haphazardly organized. I sat at the table closest to the educator and quickly befriended the three colleagues-to-be at my table. We discovered that training was arranged so that we attended class Monday through Friday from 8:30 A.M. to 4:00 P.M. for three out of six consecutive months. For the latter three months, we shadowed and paired up with an experienced $\mathrm{RN}$ on the unit to practice the theoretical knowledge learned during classes. We were told that not all of us would complete the program. Indeed, during the in-class orientation, our large group of 15 new graduate nurses slowly decreased to 12 . It seemed that training and orientation prepared us for the real world of nursing while inadvertently selecting and removing nurses who are not programmed to do the type of technical and challenging work characteristic of the ED nurse’s job. My confidence had taken a significant leap forward. If it was survival of the fittest, and I was among the 12 new graduate nurses that withstood all obstacles, then that must have meant I was fit for the job.

\section{Lectures}

To remedy some of the dry and rather boring theoretical lectures, we had the privilege of practicing skills such as intravenous insertion and venipuncture. For most of us, it was our first time, others were fortunate enough to have practiced those skills as students in the ED during their practicums. 
To encourage practice time, Laura placed three plastic baskets complete with tape, gauze, various sized needles, syringes, blood collection tubes, and some other venipuncture paraphernalia on our tables. Without resisting, some immersed their eager hands into the baskets and confidently began poking away into the rubber arm of the model used for practice, which I note, has been visibly overused by several amateurs before us. Laura promptly halted this selfinitiation, exclaiming that we were to take turns to practice and coach each other through the process of intravenous insertion. After carefully observing the experienced hands at my table, I suddenly felt unskilled. They inserted the intravenous without any trouble, as if they had done it a few hundred times before. As the fourth person in my group to practice, by then my attentive observations had taught me at minimum how to hold the needle and prepare for insertion with the correct materials gathered.

After a painfully long few minutes, I finally managed to find an appropriate size needle, I then grabbed the rubber arm, my hands were trembling and numb, I felt like I was demonstrating a skill for an instructor in class like we did for college nursing exams. As I lifted my fingers and pointed the needle into the vein to insert the intravenous I heard the person in the chair to the right of me say "you are holding it improperly, you should be inserting at a 10 to 15 degree angle, not at 30 degrees.” They sensed my anxiety and saw my nervous smile. I heard my heart beating in my ears, my face was getting hot and the inside of my mouth was dry and pasty. I was completely mortified. Laura witnessed me struggling from across the room, walked over to my table, and placed her warm hand over mine while she demonstrated the steps for inserting an intravenous catheter. In an unusually gentle and understanding voice, she explained, "find a good site, palpate the vein, clean it with a swab, bevel up, insert at a 10 degree angle, advance your catheter and retract your needle, flush, and there you have it, an intravenous catheter.” What a 
relief! My heart was still beating fast, my face cooled down, and I was able to speak again after moistening my lips. With precision and guidance I inserted my first intravenous. What a glorious moment it turned out to be.

As we approached the latter part of our in-class training we spent about two weeks on cardiac related theory and practice. We were told that cardiac complaints were common in the ED. I was proud when I realized I was gaining new knowledge and learning the unique language of the ED. I began to feel that I belonged. The terms Atrial flutter, Ventricular Fibrillation, Ventricular Tachycardia, and Normal Sinus Rhythm were just a few of the electrical rhythms of the heart that I was determined to master. In fact, I mastered them with ease, and they rolled off my tongue as they made more sense. I looked forward to the immediate future when I mastered everything there was to know about the four-chamber pump of the body. I learned beyond these complicated heart terms. I knew what they meant, but also how to respond to them.

A sudden shift of knowledge occurred for me as we had moved from covering healthcare leadership, end-of-life care, nursing issues and theoretical perspectives in nursing to understanding how to assess and respond to shock, acute stroke, cardiac arrhythmias, cardiorespiratory arrest, and even how to assist in the emergency delivery of a baby. I had such a thirst for this acute care knowledge and the ED was seemingly the perfect clinical area for me to acquire it.

\section{Susan and a Reality Check}

Three months later, 12 very knowledgeable and eager new graduate nurses started on the unit shadowing with experienced RNs. In perfect attendance, all 12 of us met Laura in the staff lounge in uniform green scrubs. Laura proudly said, “Look at you all, in your greens, so cute!” I was not aiming to look cute; I was going for more professional and smart. Laura sometimes 
makes unfiltered remarks that I was not sure how to interpret. I could have been happy that she acknowledged our attire or upset about feeling belittled. I would have to earn the professional look that I was going for, and I was certain that would happen soon.

That day, I was expected to shadow a nurse who they called seasoned; without having to ask, I could tell she had several years of experience under her belt. I was introduced to her at the nursing station. She stood against a counter leaning on her hands, relaxed and composed. She greeted me with a handshake. Without realizing how tense I was, I suddenly felt all the muscles in my shoulders neck, legs and even stomach relax. Strangely though, a part of me felt intimidated by how smart she looked.

Her name was Susan, she was in her late forties, had a powerful voice and tall personality. Susan did not wear the typical green scrubs most others did. She had on black nursing scrub pants with vertically embossed white coloured letters spelling emergency on the leg coordinated with a blue polo shirt and her stethoscope and name tag hung around her neck by a glass beaded chain. Susan’s demeanor and choice of nursing uniform exuded confidence, experience and knowledge. I wondered whether I would ever measure up to what most certainly would be "tall expectations", tall expectations that matched her tall personality.

I thought, I had been assigned to the ED Goddess, and I began to realize just how little my "training" prepared me for the realities of the ED. Just as I suspected, Susan proved herself to be efficient; I could barely keep up with her pace. I was in awe. Her confidence and speed in everything she did was enviable and mildly discouraging. My impatience, or maybe my common sense, told me it will be a long while before I could be on par with her. I was daydreaming; I mean I thought I could never be as good as her. She was the type I could idol. When Susan spoke, I nodded my head, trying to appear that I was absorbing her every word, and that I 
completely understood what she was saying. I felt like the way you would feel when someone is speaking to you and you did not hear anything they said but you nod anyway in hopes that your nod was sufficient confirmation of receipt of their message. My bobbing head seemed to make Susan feel good, that although she was speaking quickly, inundating me with information, I managed to comprehend it.

My confidence dwindled a bit when Susan said "working in the ED means you have to think fast and respond to situations quickly. You have to learn how to prioritize and work patients up fast because at times you can get four patients back-to-back and you better have them ready and assessed before the physician goes in to examine them.” I understood the full meaning of those words, that is, that was the reality of nursing in the ED, and to belong, to fit in, to stay in this work culture I would have to adjust myself to it, if I was to gain success and happiness in what is my dream career.

\section{Skilled and Independent}

Throughout the course of shadowing and orientation to the unit I had the opportunity to work with many RNs who offered varying degrees of knowledge and expertise and supported me in my learning role. With their help, I had enough time and exposure to apply all of the theoretical knowledge learned in class. I learned how to care for patients whose ages ranged from 2 days old to 102 years old. I would help relieve the pain of those with kidney stones while providing comfort to a family who learned of a new life altering diagnosis. I inserted several intravenous and performed hundreds of venipuncture and electrocardiograms. I thumped on the chest of many patients during cardiopulmonary resuscitation. I had some patients that survived while others died. I gave children Tylenol for fevers and oxygen masks for their asthma attacks. I relieved urinary retention by inserting Foley catheters to drain bladders. I relieved abdominal 
pain caused by bowel obstructions by inserting nasogastric tubes. And, well, I also medicated disruptive patients who suffered from acute psychotic episodes and behavioural issues. After three months a smaller group of ten new graduate nurses were ready to cut the cord and begin working independently with their own full patient load assignments. We were considered competent to be working alone with minimal support from senior staff.

\section{Keep Your Chin Up}

The glamourized television version of working in an ED began to dissipate and the grim realities were quickly becoming apparent to me. After consulting with my fellow new graduate RNs, common concerns began to emerge. Most of us were feeling not quite as ready to be on our own as we once imagined. There was a shared realization that previously the workload was manageable because we had ample support from senior staff. Being profoundly stressed and overwhelmed, tired, and confused were common lunch time conversations between the ten of us. When confiding in senior staff they assured us that those feelings were part and parcel of being new, were quite normal, and if we did not feel that way, then that was cause for greater concern. Hearing that kept me on my feet and more determined to work harder to overcome any hurdles. Little did I realize that there would be times in my career that no matter how fast and competent I became at performing tasks, I would still feel like I did when I first started working independently - overwhelmed, stressed, tired and confused.

\section{Challenging Experiences}

As I look back, I can recall moments in my ED career that evoked very strong emotions in me. I remember all the intricate details of those moments that made me laugh, smile, cry, experience fear, and suffer from worry for the first time. On several occasions I was confronted with patients and situations where my feelings were overwhelming helplessness, fear, sadness, 
and isolation. It is these situations that have shaped the direction of my research thesis, not my proud moments and times of success and elation. It is these stories that I must now share with you. The experience I am about to narrate has resonated with me for a long time, and imprinted a vivid picture in my mind that I will carry with me forever. It is like nothing I have experienced in the ED and was just the beginning of many more such events.

\section{Night Shift}

It was January, 2009. Tired and groggy from an afternoon nap I dragged myself into work for a night shift. It was the first night shift of my two day two night rotating shift schedule. I was now, a few months into my career, so I had a standardized routine developed for myself. I put my dinner in the fridge in the staff lounge, signed in, received report from the day nurse, and reviewed my patients’ charts. I was scheduled to work in Acute Care that night. The acute care department accommodates patients that are unstable, require close observation, or require telemetry monitoring. The maximum number of patients assigned to a nurse in this area is four. After receiving report, I began to review my patients’ charts, their diagnoses, medications, any tests that had to be completed, and outstanding physician orders that I had to process.

Less than ten minutes from the start of my shift the charge nurse gently walked over to me, stood over my shoulder to tell me that two of my four patients would be transferred to the ward. This meant that I would have two empty beds to accommodate two new patients that either walked into the ED or arrived by ambulance. I began to feel some minor stress, wondering about what was in store for me. Like a movie with alternative endings, I envisioned an array of scenarios in my head of how the next 30 minutes of my shift would play out. I thought about whether I should have assessed my sickest patient first or the two that were going to the wards at any given moment. As a new nurse, I knew that every decision I made would be scrutinized by 
the charge nurse, so I had to plan thoughtfully. I knew I could not compromise patient safety as part of my nursing standards, but concurrently I knew I had to consider efficiency and identify the best way to conduct myself in order to improve the flow of patients between departments. Within minutes a bed was available on the ward upstairs, I was notified by an alert on my computer screen. Shortly after, the porter arrived to transfer my patient upstairs to a ward. Sadly, I had not yet had time to assess that patient. I wondered and worried about whether they were stable enough to go to the ward, if there were any outstanding medications or physician orders that had not been processed by the day nurse. I felt guilty and disappointed in myself that I had not been able to enter that patient's room to say as little as hello prior to their discharge from the ED.

\section{Productivity and Time}

One thing I admire about the logistics of the ED is that in the midst of chaos there is underlying order. Every member works in collaboration and synchrony to make processes run smoothly and efficiently. Minutes after the patient was transported, cleaning staff were in the room with their mops and garbage bags. The room was cleaned, stocked and ready to house the next unwell patient. On this same particular night, my desk was at the top of a long hallway facing the ambulance bay where emergency personnel enter the hospital with patients on stretchers. Each time the doors swung open I get a draft of cool fresh air from outside and also an indication that a new patient was being brought into the ED. The triage nurse, who makes the first contact with the patient, decides on what room the patient is assigned to. While I entered my first patient's room, I once again felt a cool draft and the sound of stretcher wheels in the hallway. As I introduced myself to my patient I had to raise my voice to superimpose the louder than normal conversations that were occurring in the ambulance hallway. It was obvious that the 
paramedics and triage nurses were struggling with the new patient in the hallway. My patient looked concerned for the woman in the hallway. Her speech was audible and clear and penetrated through the walls and curtains “TAKE ME HOME! YOU SON OF A BITCH, DON’T TOUCH ME!' My patient’s eyes gazed frequently at the curtain as if he wanted me to open them so he could see the upheaval outside his room. I was curious as well, and I must

admit, a little alarmed. I apologized for the loud noises and commotion that were occurring adjacent to his barely private curtained room. Regardless of what was happening and for the sake of efficiency and time I knew I had to continue my care of this patient. What prompted me to speed up my assessment was when I overheard the charge nurse say "put her in room P.” This meant this new patient, this person who has just been swearing loudly, would be my assignment.

I was hesitant to leave the room to see what was awaiting me in the hallway. I halfheartedly opened the curtain, walked back to my desk, put my clipboard down, and looked past the plant that was sitting on top of my cluttered desk to see an elderly lady, likely in her 80s, who from a distance appeared well groomed, had all of her hair, shiny fair skin, and a medium build. For the sake of time, I strove to ignore, to mute her yelling and all the chatter of those around her while I sat in my chair and documented nursing notes on the patient I had just assessed. I realized that time was precious at that point and that if I delayed documenting I would fall behind and possibly forget the information I had just gathered in my assessment. But I would pay for this decision amongst many decisions I made that night.

\section{Ms. Levine, a Nursing Home Special}

My documentation completed, I received an abrupt report from the triage nurse who in a tense and relieved fashion extended her arm with the chart in her hand and said "here is your nursing home special for the night,” then turned around and walked away. The porter wheeled 
the elderly lady into room P. I heard her muttering something under her breath while she briskly ran the heel of her shoe into the stretcher mattress; a behavior that I sensed was odd and concerning. I wondered what it could mean. I couldn't make out what she was saying but her distress was evident, I could feel it, sense it, and her behavior immediately triggered the same feelings in me. Once her stretcher was situated I cautiously entered her room, we looked at each other. I smiled at her and said hello addressing her by her last name, Ms. Levine. Now we were in close proximity and the fluorescent lighting in the room allowed me to have a clearer look at her. She was indeed well dressed and surprisingly had all her teeth. She wore beige trousers, a pale pink shirt with a matching pink cardigan overtop and a gold Star of David pendent necklace around her wrinkly neck. She had liver spots and old bruises all over her frail arthritic hands that she had clenched onto the stretcher rails so tightly, like she thought she might be ejected if she had let go. I turned on the heart monitor in the room and got prepared to undress her when I noticed that she was staring blankly at the privacy curtains while still constantly running her shoes on the stretcher and silently grumbling nonsensical words under her breath. I continued to follow protocol, and, as I would routinely tell all my patients, I advised Ms. Levine that I would undress her, put her on the heart monitor, do an electrocardiogram, take her blood and insert an intravenous before the physician was to see her.

\section{Helpless}

As I began to touch Ms. Levine, without hesitation and in a suddenly louder than expected and slightly trembling voice she communicated with me and made it clear that I would do none of those things and that I was to take her home right then. The rails of the stretcher began to shake violently making a clinky sound caused by her even firmer grip. Her expression changed drastically, she looked angry, the wrinkles between her eyebrows were well defined, her 
lips were tightly pursed, and her feet were stiffly implanted into the stretcher mattress. "TAKE ME HOME. DON'T TOUCH ME. GET ME OUT OF HERE” is the only statements she articulated, over and over, each time in higher than tolerable decibels. I was cautious, and sensed that if I spoke another word her agitation would worsen and she would possibly attempt to leave, perhaps tip the stretcher over, and maybe fracture something, at the very least make a very big scene. Therefore, I approached her slowly and asked her in a calm voice "Ms. Levine may I have your permission to assess you by removing your shirt and dressing you in a hospital gown?” I felt a little anxious because I sensed that those words of reason, explanation that would ordinarily work to reassure other patients, would not be useful in that situation. I wondered if I was going to be able to help Ms. Levine, settle her down, de-escalate her sufficiently so that I could keep the patients flowing the way they should.

I recognized that she may have been disoriented or cognitively impaired, or perhaps experiencing a delirium, because she was elderly, from a nursing home, and did not have any insight about why she had come to be in the ED of the hospital. I know now that this lady did have significant cognitive impairments. This is based on several assessment factors I have developed skill in such as her repetitive vocalizations, poor judgment, her inability to keep track of our conversation, her difficulty answering questions, her disorientation to place, and her florid mood and behaviours. My calmer than usual technique did not, in fact, work; there was no hope for a positive reaction from her. I knew I was in trouble when she curled her fingers into her palm making a fist, and she appeared ready to do battle, to be preparing to strike me, to defend herself in some way-, from me, her nurse who only wanted to help. I realize she may have been feeling threatened by me; I must have crossed some boundary with her, maybe invaded her personal space. My hunch was to take a step back while keeping my eyes on her and pulling the 
curtain open in case I had to call for help. She continued to shout “TAKE ME HOME. DON'T TOUCH ME. GET ME OUT OF HERE”! I tried to reassure her that she would be safe and needed to see a doctor. In a hurry she struggled to climb out of the stretcher by crawling down towards the edge and pulling on the rails to give her momentum. I knew I should call for help to ensure her safety was maintained. The stretcher could have capsized at any moment and she could have struck her head, fractured her hip, I was feeling quite panicked. I was mortified that I wouldn’t be able to help this seemingly small, frail human creature, my first nursing home special.

My partner whose desk was located five feet away from mine witnessed the commotion and immediately called for help over the department microphone. Security personnel arrived in flocks. I felt helpless for Ms. Levine, and for myself. I even felt intimidated by the response, and I am a professional. How must Ms. Levine have felt? I wanted so much to help her, to prove to my colleagues that I was well equipped to care for this nursing home special, but Ms. Levine wanted nothing more than to be left alone. Now she had the attention of all these people in uniform and a doctor standing over her head who told her in a dominating tone that she was sick and needed to calm down so she could be helped. Four security personnel hovered over her, grabbed a hold of her bruised frail hands and legs, pulled her back up into the stretcher and pinned her down while the physician assessed her. The scene played out in my mind as unimaginably cruel. But, I still wondered if it was somehow justifiable, perhaps, in the ED because she was sick with a fever and diarrhea, needed treatment and did not have any decision making capacity. The responsible physician had to make decisions for her health, so we should have been able to restrain her - shouldn’t we? No one else around seemed concerned. 


\section{Powerless}

Her profane and vulgar words echoed through the ED. Her pale face was contorted, painted with rage as she spat out foamy white saliva that dripped down the side of her mouth washing away old dried up pinkish lipstick from her thin lips. It was a production, like a scene from a horror movie, "Whatever Happened to Baby Jane,” that drew a lot of attention as lay staff, visitors, and other patients stood around and gawked, watched in disbelief, yet unfortunately looked rather entertained by this unfolding scenario. In image 1 , I capture how I felt this day. I felt alone, lost and not sure how to rectify this situation.

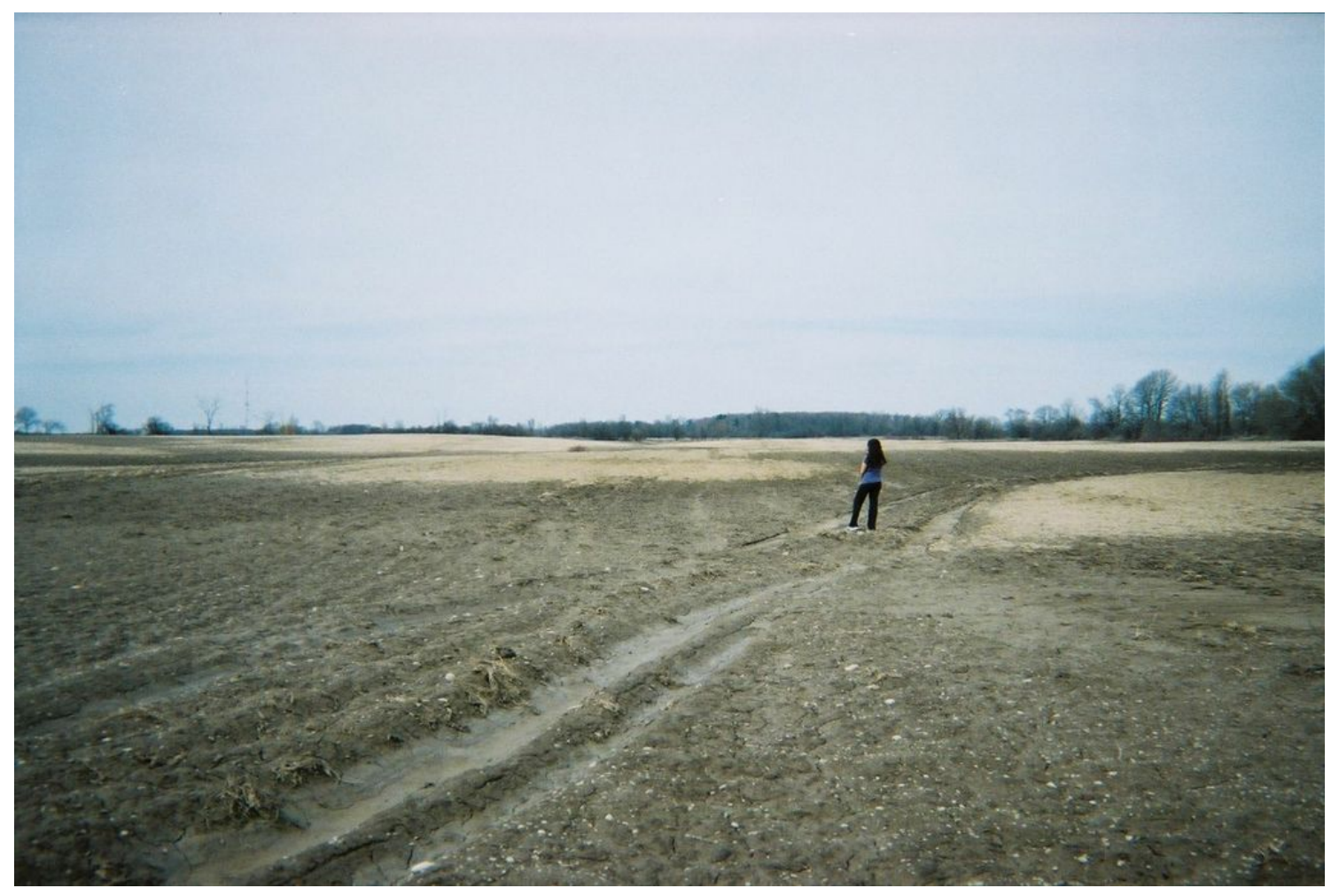

Image 1: Alone \& Helpless

I was deeply saddened, I felt powerless as I watched this woman in anguish. The entire situation was traumatizing and out of control. My uncertainty about how I could have helped Ms. Levine, how I could have communicated with her, how I could have adjusted her environment, and how I could have soothed and reassured her, had caused my patient great distress and 
provoked her to strike out and attempt to leave. As I stared at her angry face and helpless frail body pinned to the mattress I froze and deliberately distracted myself with thoughts of how I was lagging behind in all the other work I had to complete on that shift, and wondered whether my other two patients that I had not assessed yet were doing okay.

\section{Time and Efficiency}

Moments later, the physician yelled out “get this woman some Loxapine IM STAT!” I promptly brought my attention back to Ms. Levine, and it was then that I arrived at the realization that regardless of how I felt and the apparent lack of dignity and respect for this patient, we still had to function as a department and work together to move patients in and out, there was just no time for me to deliver all the tender loving care I was trained to provide in nursing school. The charge nurse made a brief appearance and offered to get the medication for me, which I graciously accepted. I was resigned to the fact, in alignment with my colleagues, that Ms. Levine should have been medicated, for her own good, and for the good of all the others in the ED.

\section{The Injection}

Unable to remove her pants, the physician ordered me to administer the medication through her clothing into her thigh muscle. I followed his instructions. What I did next meant I went against all my values and standards of patient care. I had to forcefully administer a medication while aggressively confining Ms. Levine to a mattress. While getting ready to inject the medication I blocked out all the extraneous noise and distraction in the department including the ringing of call bells, chatter of people, and alarming of telemetry monitors. With my barely steady and clammy right hand I used a 90 degree angle and stabbed the needle into her outer right thigh. I felt crunching of the pants and skin. I slowly ejected the medication through her 
pants and into her vastus lateralis. It sickened me to feel the crunching again this time after withdrawing the needle out of her muscle, skin and then pants. Mechanically, my emotions shut down; I clamped the safety port of the needle and discarded it in the sharps container next to the bed. I looked up at the patient and found her looking back at me with fierce eyes. Speaking ever so audibly and slowly, annunciating every syllable of each word she called me a "night shifting whore.”

\section{Second Reality Check}

It felt as though every last patient I have ever or will ever care for has punched me in the stomach. I was distraught, feeling I had enacted that ethical dilemma they teach you to avoid in nursing school. The feeling sunk deep into the pit of my stomach, down to my very soul. It isn't that Ms. Levine’s choice of words had insulted me. I wasn't bothered by what she had called me. It was more that I felt I had failed her humanity, because I know very well that she suffered from a profound incapacity to protect herself. Her only self-defense was to try and hurt me with her words. When I had a chance to review the usage of the medication I administered I learned that I gave her an antipsychotic medication used to treat conditions such as schizophrenia. I reviewed her nursing home records and read that she had a diagnosis of Alzheimer's disease. I wondered if her behaviour was a result of Alzheimer's or was it just part of being old. I didn't know the answer to this, I had never thought about it before. I did not know that I would ask myself this question over and over, with other cases to follow.

\section{Who's Responsible?}

The emotional aftermath of that experience resonated with me for many shifts after that night. I realized I had impinged on Ms. Levine’s human rights. I knew I did not advocate for her as I should have, and most importantly I had gone against my personal code of ethics wherein I 
practiced in an evidence-informed way, that met standards of practice and resulted in high quality and safe patient care - always. In retrospect, I wonder why my six month orientation didn't include training on how to manage and cope with situations such as this. If it had, would I have responded differently? Would the outcome have been less painful for Ms. Levine, and for me?

I promised myself that if I ever had an opportunity to do so, I would look for some answers. I would find a way to share my experience with others and learn about theirs. Surely, I couldn't be the only emergency nurse who had experienced the difficulties inherent in our jobs. As long as there are emergency patients with Alzheimer's and difficult-to-manage situations, that we produce or are presented with, there is a possibility of encountering moments such as the one I've experienced. It is important to reveal these issues and begin to understand the dilemmas nurses, and their patients, confront every day. Upon reflection of this event, even though an intramuscular administration of a neuroleptic may have been warranted as a necessary intervention in Ms. Levine's particular case, my feelings remain that the management of this woman was inappropriate. Ms. Levine should have been assessed and managed with more dignity. She should have been carefully examined for possible triggers of her behaviour, gestures and movements such as pain, infection, discomfort, fear, hunger, and even toileting needs. Perhaps an order of antibiotics or analgesics or even a sandwich would have sufficed to make Ms. Levine more comfortable. These more thoughtful and person-centred assessments were not considered in Ms. Levine’s case, rather medical interventions took precedence.

In the next chapter I provide a background of dementia, including its prevalence and incidence, its significance to research, the purpose of this study, and the research question. 


\section{CHAPTER 2}

DEMENTIA IN THE EMERGENCY ROOM: A SILENT CRISIS

BACKGROUND, LITERATURE REVIEW \& SYNTHESIS, RESEARCH SIGNIFICANCE, STATEMENT OF STUDY PURPOSE, RESEARCH QUESTION 


\section{Background}

Similar to Ms. Levine’s situation, EDs serve as the main portal of entry to hospitals for persons requiring health care services (Chan, Schull \& Schultz, 2001). A report by the Canadian Institute for Health Information (2010) found that those 65 years of age and older used EDs more than any other age group (Appendix A). According to data from the Institute for Clinical Evaluative Sciences (2011) adults over 66 years of age with a diagnosis of dementia utilized health care services more than those without a dementia diagnosis (Appendix B). In Ontario, there are approximately 1.8 million seniors, and of these 250,000 meet the criteria for dementia (Canadian Mental Health Association, n.d.). In Canada, one in eleven people over 65 years of age has a diagnosis of dementia (Alzheimer’s Society of Canada, 2010). The prevalence of dementia increases with advancing age, rising $28 \%$ for those 85 years and over (LHINsAlzheimer Society of Ontario, 2007).

Dementia is an umbrella term for a variety of age-related disorders that affect the brain. The most common type of dementia is Alzheimer's disease, also known as Alzheimer's dementia (Gililland, 2007). Alzheimer’s dementia is characterized by irreversible degenerative changes in the brain as a result of changes in the function of two proteins, beta-amyloid and tau, which are part of plaques and neurofibrillary tangles that form in place of healthy brain tissue resulting in progressively worsening capabilities related to basic skills, speech, thought process and movement (Gililland, 2007). The second most common type of dementia is vascular dementia (Gililland, 2007). Vascular dementia is caused by blood circulation problems in the brain. Multiple cerebral vascular incidents occur, causing oxygen deprivation resulting in brain tissue death and subsequently impaired cognitive ability (Sander, 2002). Another common type of dementia is Lewy Body dementia. Like Alzheimer’s dementia, Lewy Body dementia results in 
progressive mental and physical decline. It is characterized by fluctuating periods of confusion and alertness, visual and auditory hallucinations, and Parkinson's type motor deficits such as rigidity and spontaneous movements (Gililland, 2007).

The growing prevalence of dementia and its associated behavioural problems along with the incidence of ED usage by these patients may challenge nurses working in this area. As my narrative has also shown, providing care can be difficult when nurses have to manage the physical and responsive behaviours of dementia in addition to the responsibilities associated with routine nursing care (McCloskey, 2004). The focus of care in the ED is meeting acute health needs of individuals in a time sensitive manner. Any attempt to interpret and manage responsive behaviours can be time consuming and require careful attention to interpersonal relationships (Moyle, Olorenshaw, Wallis, \& Borbasi, 2008). Therefore, understanding emergency nurses' experiences of caring for patients with dementia is an important area of research.

\section{Literature Review and Synthesis}

Current literature that focuses on dementia care in general hospital wards predominantly addresses nursing practice issues related to assessment and management strategies of dementiarelated behaviours (Andrews \& Christie, 2009; Borbasi, Jones, Lockwood \& Emden, 2006; Cunningham \& Archibald, 2006; Crabtree \& Mack, 2010; McCloskey, 2004; Moyle et al., 2008; Tolson, Smith, \& Knight, 1999., 1999; Weitzel et al., 2011). An advanced electronic literature search was performed through the information retrieval services of CINAHL, Pubmed, and Medline Ovid in order to locate relevant literature related to nurses' experience with dementia. First, an advanced search was conducted in CINAHL using the major concepts of 'emergency nurses experience' with the Boolean operator 'AND' 'dementia care.' This resulted in 3415 articles that were limited to English language and academic peer-reviewed documents, and to 
keep with more recent literature a limitation included published literature between the years 2000 and 2013. To narrow search results to more relevant articles the key terms were changed to 'emergency nursing' with the Boolean operator 'AND' 'older patients.' This search returned 12 English language peer-reviewed articles. The abstracts of these documents were reviewed for appropriateness and one article that sought to investigate the nurses' experiences in caring for older hospitalized patients (Kihlgren, Nilsson \& Sorlie, 2004) was retrieved. To find documents more closely related to emergency nurses’ experience in caring for patients with dementia another search was performed using Medline Ovid and Pubmed. In Medline Ovid the key terms 'emergency nursing' and 'dementia care’ were searched joined with the Boolean operator 'AND'. Using similar search limitations a total of eight documents were located, the titles were reviewed and none were found to relate to the nurse's experience. Instead, they focused on the experience of patients, family caregivers, and Personal Support Workers in Long Term Care Facilities.

Finally, a search was conducted in Pubmed using a variety of key terms. First, 'nursing' and 'dementia care experiences' were searched with the Boolean operator 'AND.' The search was limited to full text English language documents published between the years 2000 - 2012. The titles of 192 resulted articles were scanned, and two articles (Borbasi, Jones, Lockwood, Emden 2006; Eriksson \& Saveman, 2002) that specifically related to nurses’ experience with dementia in an acute care setting were retrieved. In order to retrieve articles pertinent to challenging experiences in caring for patients with dementia another search was conducted in Pubmed using key terms of 'nurses' and 'dementia care challenges.' These terms were again linked with the Boolean operator 'AND.' The abstracts of 40 documents were scanned and two studies which focused specifically at nurses' experience with patients with a dementia diagnosis 
in a general hospital setting (Chang et al., 2009; Fessey, 2007) were retrieved and included in the literature synthesis. Combined, a total of five articles from three databases, which related directly to the nurse's experience with dementia care in an acute care setting, were selected for synthesis. In the next section, I provide a synthesis of literature related to: a) significance of dementia care in the ED; b) challenging and responsive behaviours in dementia; c) culture change and dementia care; d) person-centred behavioural management; e) the influence of environment on dementia care; and f) staff development in dementia care.

\section{Significance of Dementia Care in the ED}

The impetus for the proliferation of studies on the assessment and management of patients with dementia can be explained in part due to the high global incidence and prevalence of hospitalized elderly individuals who have an underlying diagnosis of chronic dementia (Cunningham \& Archibald, 2006; Moyle et al., 2008; Weitzel et al., 2011). In Canada, approximately 500, 000 people are living with a diagnosis of dementia (Alzheimer Society of Canada, 2010). This is also true for population estimates in other countries. For example, in the United States 5.2 million people have a diagnosis of dementia (Weitzel et al., 2011); in the United Kingdom 870, 000 people are living with dementia (Cunningham \& Archibald, 2006), and in Australia 250, 000 people are living with dementia (Moyle et al., 2008).

Elderly patients with an underlying diagnosis of dementia accessing the health care system through EDs for acute care needs typically exhibit one or more comorbidities, heightening the complexity of their health care needs (Kihlgren et al., 2004; McCloskey, 2004). A synthesis of literature reveals that emergency nurses are ill-prepared to provide optimal care to persons with dementia. Common barriers include nurses difficulties in managing behaviours associated with dementia such as aggression, agitation, and wandering (Bianchetti \& Trabucchi, 
2004), a lack of knowledge of the assessment and care of people with dementia, and working in hospital environments that are not conducive to the needs of elderly people with dementia (Andrews \& Christie, 2009; Cunningham, 2006; Cunningham \& Archibald, 2006; Crabtree \& Mack, 2010). Part of this lack of knowledge is related to the behaviours associated with dementia.

\section{Challenging Behaviours in Dementia}

Behavioural and psychological symptoms of dementia (BPSD) have been defined as a diverse group of symptoms and behaviors (Cerejeira Lagarto, \& Mukaetova-Ladinska, 2012) causing disturbances in perception, thought content, mood, motor activity, altered personality traits, and behaviour (Cerejeira, et al., Bianchetti \& Trabucchi, 2004; Finkel, Costa e Silva, Cohen, Miller, \& Sartorius, 1996). These symptoms were typical of how Ms. Levine presented to me in the ED, confirming that she was likely experiencing BPSD. In a sample of 148 patients with dementia in the community, 63.7\% showed five or more BPSDs (Bianchetti \& Trabucchi, 2004). Psychological manifestations of dementia include hallucinations, delusions, misidentification, anxiety, and sleep disturbances (Bianchetti \& Trabucchi, 2004). Behavioural disturbances in dementia commonly include but are not limited to aggression, agitation, and wandering (Bianchetti \& Trabucchi. 2004; Finkel et al., 1996). Of all the features of dementia, the behavioural responses have shown to result in the greatest amount of distress and burden for caregivers (Cipriani, Vedovello, Nuti, \& Di Fiorino, 2011; Claudia \& Arthur, 2003; Sourial, McCusker, Cole, \& Abrahamowicz, 2001). In the literature, these behaviours have been defined as challenging and difficult to manage by practitioners and family caregivers (Bianchetti \& Trabucchi, 2004; Cipriani et al., 2011). It is apparent from my own personal narrative that I struggled with these behaviors and was ill-prepared how to best manage them. 
Agitation is a broad concept often used interchangeably with aggression in dementia related literature (Purandare \& Burns, 2000). Agitation is behaviour associated with verbal, vocal, or motor activity common in individuals with dementia and can be divided into four subtypes. Based on the Cohen-Mansfield Agitation Inventory these are: physically non aggressive behaviours (restlessness, pacing, mannerisms, hiding things, inappropriate dressing or undressing); physically aggressive behaviours (hitting, pushing, scratching, biting, kicking, and grabbing); verbally non-aggressive behaviours (negativism, repetitions, interruptions, constant requests for attention); and verbally aggressive behaviours (screaming, making strange noises, cursing, and temper outbursts) (Cohen-Mansfield, 1997). Behavioural agitation associated with dementia is reported to occur in between $30 \%$ to $50 \%$ of cases (Cipriani et al., 2011; Sourial et al., 2001).

Behavioural symptom management was an area identified in the literature as challenging by general practitioner nurses, palliative care speciality staff, and aged or dementia specialist health-care practitioners (Chang et al., 2009). In this study, participants reported a lack of knowledge and skill related to managing physical and verbal aggression among patients with dementia. I share a common experience with the nurses from this study. It was not the inherent time management or patient assessments I struggled with that particular day in the ED; rather I struggled with managing the BPSD of Ms. Levine. I simply followed the orders of a physician without having knowledge and skill of my own on how to prevent or alleviate Ms. Levine of agitation. My experience is also mirrored in another study of five acute care wards including one ED, wherein nurses reported having to use force, abuse and neglect to manage patients and reported being afraid of individuals with dementia when they had outbursts of rage as they would become extremely strong (Eriksson \& Saveman, 2002). Sourial et al. (2001) conducted a study in 
an acute care hospital about agitation in patients with dementia. They found that verbally agitated behaviours and physically non-aggressive behaviours were always a statistically significant correlate of staff burden regardless of the number, frequency, or disruptiveness of behaviours. These behaviours occurred in $94.6 \%$ of patients with dementia during a two week period (Sourial et al., 2001).

Wandering and other related activities such as rummaging, pacing, leaving a place of care such as an institution against advice, getting lost, losing one’s way, getting separated and reduced nutritional intake and rest have been reported as some of the most challenging and troublesome behaviours to manage outside of the ED, for caregivers and professionals alike (Colombo et al., 2001; Dewing, 2005; Lai \& Arthur, 2003). In the acute care study by Eriksson and Saveman (2002), nurses faced ethical situations when they had to administer high doses of sedative or hypnotic medications to patients against their will in order to make it possible to manage wandering situations and still have time to care for other patients. In the same study, nurses expressed frustration as a result of wandering behaviour and its disturbing influence on other patients (Eriksson \& Saveman, 2002). In a study of inpatients from a Special Care Unit, for patients with dementia, Colombo et al. found that $51 \%$ of the sample engaged in wandering. There is an assertion that wandering occurs as patients with dementia seek to find a place of safety and familiarity (Lai \& Arthur, 2003). The behavioural and psychological symptoms of dementia described vary in nature and frequency and from person to person based on the individuals' environment, their needs, and whether those needs are addressed by nursing staff (McCloskey, 2004; Weitzel et al., 2011) 


\section{Culture Change and Dementia Care}

In more recent dementia related research there is an evident shift in thinking and language used when discussing challenging and behavioural and psychological symptoms of dementia. According to research by Dupuis, Wiersma, and Loiselle (2012), current language and approaches to manage behaviours in dementia are “pathologizing” (p. 164). For example, behaviours identified as violent, aggressive and challenging are interpreted from a pathophysiological and psychological model of illness and disease. These behaviours are seen as the result of disease progression in the context of dementia. Dupuis et al. assert that psychological models often overlook the meaning of behaviours and link behaviours to psychiatric disorders that are inappropriately treated using pharmacological means. Based on these predominant discourses individuals are labelled and thereby become defined as their misunderstood behaviours: the wanderer, the fighter, or the resister of care. Dupuis et al. assert that how we label people ultimately influences our decision making in how we respond to them.

In their study of 48 long term care staff, Dupuis et al. (2012) demonstrated that the predominant biomedical discourse impacted the meaning that staff attached to behaviours and consequently how they responded to the pathology in these behaviours. In the case of Ms.

Levine, her behaviours were labelled as disruptive and negatively impacting the ED principles of time and efficiency. Because of this notion a tranquilizing medication was thought to be the most favorable intervention. The difficulty with contextualizing behaviour more mindfully to larger social and environmental circumstances has implications for individuals with dementia who continue to be misunderstood or mistreated. Dupuis et al. note that a more person-centred approach to understanding behaviour is needed in dementia care. This begins with changing the way we understand and discuss dementia related behaviours. Dupuis and colleagues adopt and 
encourage the use of "responsive" behaviours as an alternative discourse and conceptualization of behaviour in the context of dementia. This more holistic discourse views all actions as meaningful and shifts current practice from blaming and "pathologizing" to recognizing that all behaviour has purpose and meaning. In Ms. Levine's case, her behaviour in the ED could be given an alternative meaning: striking out because she was asked to undress, she was restless and

frustrated. Given the movement towards more person-centred language in the dementia context, I will be using the term “responsive” behaviour (Alzheimer Society, 2012; Dupuis et al., 2012) when referring to challenging behavioural and psychological symptoms of dementia.

\section{Person-Centred Behavioural Management}

Embedding principles of person-centred care in behavioural management of dementia is required to meet best-practice standards. As in Ms. Levine's case, a common approach in the management of responsive behaviour currently includes the administration of psychotropic drugs, such as Haldol or Loxapine, which are typically used to treat psychotic disorders such as schizophrenia and bipolar disorder. Utilizing non-pharmacological methods as an alternative to the current medical treatment of responsive behaviours ensures persons with dementia receive the best possible care (Alzheimer's Society, 2011). The philosophical underpinnings of personcentred care are an important consideration when planning interventions to manage responsive behaviours. The principles of trust, understanding, respect and individualism should be considered when planning care of patients with dementia (McCormack \& McCance, 2010).

According to the Alzheimer's Society of Canada (2012) people with dementia should be treated as individuals, whose care should be tailored to their unique needs based on their habits and desires. Interventions are aimed at supporting the individual to their maximum potential and concurrently involving them as partners in their plan of care. A standardized tool can be used to 
learn more about the person with dementia and their background, their likes and dislikes, culture, life experiences, and any unmet needs such as pain, boredom or under stimulation that may be provoking responsive behaviours. Then interventions can be targeted at addressing any of the findings (Elliot, 2013). One such assessment tool is known as P.I.E.C.E.S. (MAREP, 2005). P.I.E.C.E.S is an acronym that provides a systematic framework for understanding the person with dementia. The person's Physical, Intellectual, and Emotional self, and their Capabilities, Environmental, and Social factors are considered when assessing the patient with dementia (MAREP, 2005).

Another model that can help explain antecedents of responsive behaviours is known as the Progressively Lowered Stress Threshold (PLST) (Hall et al., 1987). This model has been used to help identify explanations for responsive behaviours that are related to the person's personal, social, and environmental factors (Smith, Hall, Gerdner, \& Buckwalter, 2006). The PLST model suggests that four clusters of responsive behaviours are recognizable in dementia. Three of them are related to the cognitive changes found in dementia, while the fourth is related to PLST, which includes behaviors that emerge when environmental demands, external or internal, exceed the person's ability to cope and adapt (Smith et al., 2006). According to this model, persons with dementia are less likely able to manage stress as the disease progresses, that is, their stress threshold is reduced. In order to reduce the stress, they may attempt to leave, or become anxious. Triggers of stress can be related to various things such as hunger, fatigue, multiple stimuli, discomfort, and illness (Smith et al., 2006).

The PLST model contains six principles of care that allows individualized nonpharmacological interventions to be selected that will keep stress at a manageable level. In short, these principles include: maximizing the individuals functioning by supporting losses; providing 
unconditional positive regard; using behaviors to gauge activity and stimulation levels; teaching caregivers to listen to behaviors; modifying the environment to support losses; and providing ongoing assistance to formal and informal caregivers. The PLST model provides a comprehensive framework for assessing and responding to the needs of persons with dementia (Smith et al., 2006).

Various activities providing social, physical and cognitive stimulation can be used to reduce the likelihood of responsive behaviours and minimize the use of chemical and physical restraints (Cohen-Mansfield, Libin, Marx, 2007; Cohen-Mansfield \& Werner, 1997). These include cognitive stimulation therapies such as: reminiscence groups, which involves the use of past memories to generate interest; validation therapy, which accepts the truth of another's experience, commonly used for person's with advanced dementia; reality-orientation, which in some instances can be appropriate to remind patients with dementia about the time, place and person factors of their environment; social interaction and pleasure in patients with dementia, while also engaging the person in meaningful activities such as word and number games and current affairs; alternative therapies such as: music therapy, companionship with pets, art, and relaxation techniques; and social and diversional activities which include physical exercise and entertainment (Cohen-Mansfield, Libin, Marx, 2007; Cohen-Mansfield \& Werner, 1997; Thompson \& Hoe, 2010).

At times, symptoms of delirium can be confounded with those of dementia, and if not appropriately differentiated, any efforts at addressing responsive behaviour may not be effective (RNAO, 2003). Unlike the progressive decline of cognitive function that defines dementia, delirium is an acute change in mental status from baseline functioning, it is transient in nature, fluctuates in intensity, and is common among hospitalized patients (Shapiro \& Mervis, 2007; 
RNAO, 2003). While delirium can superimpose dementia, the symptoms of delirium are considered a medical emergency brought on by some stimulant during an individual's hospitalization (Shapiro \& Mervis, 2007). Causes of delirium may include: infections, dehydration, poor nutritional status, constipation as well as use of certain drugs such as anticholinergics, painkillers, sleeping agents and anaesthetics; as such, the underlying cause must be addressed (Richards, 2011). Therefore, a comprehensive assessment is required when addressing delirium in older hospitalized people. It is said that a diagnosis of delirium is missed in up to $70 \%$ of cases (Richards, 2011). The Confusion Assessment Method Instrument (CAM) is designed to identify individuals experiencing delirium (RNAO, 2003). This tool assesses whether an individual meets three of the following four criteria: acute onset of change in mental status, inattention, disorganized thinking, and an altered level of consciousness, in which case they are experiencing a delirium (RNAO, 2003).

In order to overcome some of these challenges, healthcare organizations can adopt formal programs aimed at assisting staff and improving the patient experience. One such program is the Hospital Elder Life Program (HELP) (Inouye, Bogardus, Charpentier, Leo-Summers, Cooney, 2000). The HELP is a comprehensive model of care for older people who are hospitalized. This program is delivered by a skilled interdisciplinary team with expertise in the care of older people. The goal of this model is to maintain physical and cognitive functioning during hospitalization, maximize independence at discharge, assist with transition from hospital to home and prevent unplanned readmissions (Inouye et al., 2000). In a hospital setting all older persons would be screened, and those at risk would be identified and included in the program. Identifiable risk factors include: cognitive impairment, vision/hearing impairment, immobilization, psychoactive medication use, dehydration, and sleep deprivation. Then evidenced-based interventions would 
be selected for the patient by an Elder Life Nurse Specialist based on the identified risk factor. Some interventions include: implementing therapeutic activities, reality orientation, early mobilization, non-pharmacological approaches to sleeping/anxiety, early recognition of dehydration, and noise reduction strategies. According to Inouye et al. (2000) a study on the use of HELP across various hospitals in North America has shown to improve patient outcomes, enhance patient and family satisfaction, provide nursing education, improve quality of care, and

provide cost-effective care. In addition to implementing HELP and other models mentioned, the person's environment should also be assessed as this may contribute to responsive behaviour.

\section{The Influence of Environment on Dementia Care}

Comfort and safety for the patient with dementia is often achieved through familiarity of people and places, routine of activities, consistency of caregivers, and peace and calmness of the environment (Borbasi et al., 2006). The ED environment is not designed to accommodate the unique needs of elderly patients with dementia (Borbasi et al., 2006; Eriksson \& Saveman, 2002; Hwang \& Morrison, 2007; Moyle et al., 2008). In the ED setting, patients with dementia are exposed to environments that are not familiar. These environments are busy and noisy with constant sounds of bells, technical equipment, announcements and activities associated with the healthcare needs of other patients and their visitors, all of which can be over-stimulating for the patient with dementia leading to stress and agitation. In retrospect, Ms. Levine’s behaviours may be a result of being brought from a place of comfort with people she trusted to an ED setting that was unfamiliar. Borbasi et al. in their qualitative, descriptive study about the care of patients with dementia, conducted semi-structured interviews with 25 health care professionals across three large teaching hospitals in Australia to explore, understand, and interpret nurses' and health care professionals' experiences of managing patients who have dementia and have been admitted to 
hospital for treatment of a non-dementia-related illness. Five major themes were identified as having influence directly or indirectly on how people with dementia are managed. The Acute Care Built Environment was a significant theme that spoke to the structure and nature of the environment. Patients with dementia were said to be reassured by routine and familiarity. When these patients were approached by multiple, unfamiliar caregivers and a change in their physical environment and daily routines, it resulted in unpredictable behaviours such as aggression. The availability and involvement of family members during hospitalization of the person with dementia is paramount. Having support from family caregivers during patient's hospitalization can ensure the safety and comfort of the patient are maintained (McCloskey, 2004). Information provided by family during the patient's physical assessment, gathering of health history, and treatment decisions can be helpful when planning the individualized care of the person with dementia (Borbasi et al., 2006; McCloskey, 2004; Moyle et al., 2008).

ED practices are dominated by the biomedical model of care, which emphasizes and prioritizes medical knowledge and technical skills (Cunningham \& Archibald, 2006; Eriksson \& Saveman, 2002; Hwang \& Morrison, 2007; Kihlgren et al., 2004). According to a review by Hwang and Morrison, the ED is designed with the intent of quick patient turnover, high throughput, and maximal use of resources; these design features are not aligned with the care required by elderly patients, especially those living with dementia. This implies that nurses are responsible for a greater number of patients and have less time to adequately meet all patient needs (Cunningham \& Archibald, 2006). In a phenomenological study to describe nurses' experiences of difficulties related to caring for patients with dementia, Eriksson and Saveman (2002) interviewed 12 nurses across five acute care settings. The majority of participants in this study reported a constant demand from the top of the organization to reduce care time and 
increase throughput of patients, which impeded their ability to communicate with patients and meet their unique needs. In a qualitative, descriptive study by Kihlgren et al. on what constitutes good nursing care for patients over 75 years of age in an ED, the researchers interviewed ten emergency nurses to find that nurses' ability to instill feelings of trust in their patients with dementia is prevented when medical care and practical activities supersede other aspects of care,

such as time spent communicating and trying to understand patients' needs. The key to managing the ED experience for a patient with dementia lies in understanding person's needs and responding to them in a skillful and knowledgeable manner (Cunningham \& Archibald, 2006).

\section{Staff Development in Dementia Care}

Several authors have noted that professional practice development is inadequate in the area of dementia care for those who are employed in the acute care sector (Andrews \& Christie, 2009; Crabtree \& Mack, 2010; Moyle et al., 2008). As I reflect on my orientation, I received no education about dementia care in the ED. In a research-based literature review between 1986 and 2006 related to the management of people with dementia in the acute care setting, Moyle et al. found consistent evidence that nurses lack clinical understanding of dementia and are illprepared to assess or manage patients with dementia. This includes identifying triggers of responsive behaviours, understanding the emotions behind behavioural responses, and responding appropriately (Chang et al., 2009; Cowdell, 2010). It is understood that a lack of knowledge about the needs of patients with dementia hinders the provision of optimal care (Moyle et al. 2008). One way to assess a patient's needs is through effective communication. This can be challenging and stressful for nurses looking after patients with dementia, as it requires knowledge, skill and patience (Cunningham, 2006; Weitzel et al., 2011). Patients with cognitive impairment have difficulty with comprehension of information, concentration and self- 
expression, as well as problems with memory, word finding and visual perception, all of which compromise the individual's ability to communicate, resulting in increased time needed by ED nurses to interact with their patients (Cunningham \& Archibald, 2006; Hoe \& Thompson, 2010).

The literature suggests that in order to enhance communication, the health care provider should give the patient full attention, not rush conversation, remain at eye level contact, communicate in a quiet environment, address the patient by their name, and use short, simple, specific sentences (Cunningham, 2006; Cunningham \& Archibald, 2006; Weitzel et al., 2011). Moyle et al. (2008) recognize that this type of communication requires effort and time, which may not be ideal with the ED nurses' competing work demands. Compounding the issue is that the specific adaptations required for enhanced communication in the dementia context are most often not taught in ED orientation programs. As mentioned, formal tools and models such as P.I.E.C.E.S, PLST, and HELP can be incorporated in training and staff development initiatives to increase familiarity and comfort during care interactions of patients with dementia.

\section{Significance of Research Project}

As previously mentioned, studies undertaken to describe the experience of nurses caring for patients with dementia specifically in an ED are scarce. A literature search resulted in only five studies that sought to investigate nurses’ experiences of caring for older hospitalized patients (Kihlgren, Nilsson \& Sorlie, 2004) of which two studies looked specifically at patients with a dementia diagnosis in a general hospital setting (Chang et al., 2009; Fessey, 2007) and two in

acute care settings (Borbasi, Jones, Lockwood, Emden, 2006; Eriksson \& Saveman, 2002). In an Australian based study, Chang et al. (2009) utilized action based research to understand challenges for professional care of advanced dementia. They conducted focus groups with five groups of professionals including dementia care specialists, volunteers, volunteer managers, 
palliative care specialists, and general practitioners from residential aged care facilities.

Although the findings were invaluable, the study was centred on the needs and deficits in service delivery related to general care needs, rather than the experience of nurses in dementia care. Other challenges might have emerged had the authors asked other questions related specifically to the professional experience of caring for people with dementia. In addition, views were obtained from many disciplines; however, ED nurses were not represented in the study sample. In another Australian based study, authors Borbasi et al. (2002) interviewed an interdisciplinary group of practitioners to explore health professionals' perspectives of providing care to people with dementia in the acute setting. Their study did yield rich information about the features of a dementia-specific environment and how management can be supportive in dementia care as well as the logistics of the environment. However, given the lack of representation of ED nurses, there was no discussion about the experience of nurses in acute care.

Other studies related to nurses' experiences have been conducted, however with limitations in their findings. For instance, in a Swedish based study, authors Kihlgren et al. (2004) conducted two interviews with ten ED nurses and asked participants to discuss a situation where they felt an older patient received good care. This question, although important, does not attempt to explore any other facet of nursing experience except for the good care patients received. The study does allude to the experiences of nurses with dementia care; however, neither the study question nor discussion focus primarily on the experience of nurses related to patients with dementia. In another Swedish based study by Erikson and Saveman (2002) the authors described nurses' experiences of difficulties related to caring for patients with dementia in acute care settings. The findings from this study are based on the experience of the 
phenomenon from a sample of 12 acute care ward and ED nurses; therefore, among the literature located, it is the most closely related to the proposed study.

As the main portal of entry for health care services, the ED typically assumes responsibility of older patients with dementia during their most acute stage of illness. This may involve being admitted for acute stroke, fracture or infection. The combination of an acute illness with dementia places additional responsibility on ED nurses (Kihlgren et al., 2004). Furthermore, the ED can be over stimulating for the patient with dementia because of the urgent nature of this environment. It has excessive lighting and complicated structures and is typically noisy due to the technical equipment used and the high traffic of visitors and patients (Nystrom, Dahlberg, \& Carlsson, 2003). As a result of this, the patient with dementia may exhibit responsive behaviours such as aggression or agitation, adding additional stress to nurses’ workload.

The urgent characteristics of the ED environment coupled with the demand on nurses and the unique needs of the older person with cognitive impairment makes understanding the emergency nurse’s experiences imperative. Furthermore, I was unable to locate Canadian initiated studies. Study results may vary depending on the larger sociocultural and political context in which participants and their experiences are geographically situated. Therefore, this study is intended to minimize the gap in knowledge by exploring the Canadian ED nurses experience with the study phenomenon.

\section{Statement of Study Purpose}

The purpose of this narrative study is to understand the everyday experiences of emergency nurses through their stories as they care for older people living with cognitive impairment. The focus is to explore their narratives in the provision of care to acutely ill elderly 
patients with an underlying diagnosis of dementia who present with responsive behaviours in the ED.

\section{Research Question}

The central question of interest to this study is: How do emergency nurses narrate their experiences of caring for patients with responsive behaviours due to an underlying diagnosis of dementia? A sub-question of this study is: How do dominant discourse and power relations between nurses, their work environments and the healthcare system shape nurses’ stories and their practice of caring for this population? 


\section{CHAPTER 3}

THEORETICAL LENS, METHODOLOGY, AND DESIGN 


\section{Theoretical Lens}

I chose Critical Social Theory (CST) as the theoretical lens to guide the process of this study (Creswell, 2007). CST challenges commonly accepted norms within society that have been imposed and maintained by dominant groups who hold power (Sumner, 2010). Browne (2000) suggests the most significant contribution of CST to nursing may be in challenging the ideological assumptions that drive nursing science. CST assumes that every form of social order entails some degree of domination and power (Browne, 2000). By critiquing the social order, CST can serve as a catalyst for emancipation by transforming normative assumptions and exposing power relations imposed by dominant groups (Browne, 2000).

The dominant model of care delivery is the medical model in today's efficiency-driven health care organizations (Nystrom et al., 2003). As I have recognized, the assumption held is that curing a patient takes precedence over caring, wherein prioritizing medical procedures and practical tasks is valued over quality nursing care (Nystrom et al., 2003). As emergency nurses, we often find ourselves in a state of tension between the kind of care that is required by our standards of practice and meeting the needs of the organization (Kihgren et al., 2004). Kihlgren et al. explain that to meet the needs of elderly patients, nurses require time and resources, which is incongruent with today's health care system.

Nurses are pressured to provide ethical care under time, resource, and knowledge limitations. In my study, nurses’ stories have been elicited to examine power relations and uncover inequalities. Sumner (2010) eloquently stated "the researcher becomes a 'socio-cultural analyst' ... dissecting the ways people connect their everyday experiences to the social representations of such experiences” (p. 160). Thus, CST offers this study a critical lens to analyze and gather stories related to nurses' experiences by critiquing social order, knowledge, 
language, meanings, values, culture, and power relations inherent in their experiences (Browne, 2000). My own personal narrative that I recounted in chapter one provides beginning insight into how power relations and dominant structures influence nurses' attitudes and behaviors and how they influenced my relationship with Ms. Levine and my nursing practice. As I recall, these relations, influence of peers and structures altered and restricted my practice, leaving me at a loss with my first nursing home special.

\section{Research Methodology}

\section{Method}

The origins of narrative research are in the humanistic sciences including philosophy, anthropology, sociology, and psychology (Lieblich, Tuval-Mashiach, \& Zilber, 1998). Narrative research is a relatively new research methodology in the social sciences that focuses on stories and storytelling (Clandinin \& Connelly, 2000). Narrative research and CST share a common goal of consciousness-raising allowing participants and researchers to find deeper meaning and understanding of themselves and others (Bruce, 2002).

In this study, I employed a narrative methodological approach, drawing from the work of Lieblich, Tuval-Mashiach, and Zilber (1998) to explore and understand the experiences of emergency room nurses who care for patients with dementia. This approach was selected because of the authors' theoretical position that by studying personal narratives the researcher can access not only individual identity and its systems of meaning, but also the meaning of narratives within a culture and social world (Lieblich et al., 1998). In this study the goal is to understand the personal experiences of emergency nurses and how the dominant discourse and power relations of work environments and the healthcare system have shaped their experiences and stories. According to Lieblich et al. narrative research refers to any study that uses or 
analyzes narrative materials, such as data which is collected as a story provided in an interview. The narrative approach is situated within the qualitative paradigm which espouses pluralism, relativism, and subjectivity in human reality (Lieblich et al., 1998). In this approach, learning that is accomplished through individual's verbal accounts about their lives and their experienced reality is valued. Individuals' stories shared are not complete and accurate representations of reality. They are constructed around core facts or life events that represent individuality and creativity in selection, addition to, emphasis on, and interpretation of these "remembered facts" (Lieblich et al.,1998).

A story recorded during an interview and transcribed is influenced by the context within which it is narrated. The story gathered is only one account of the possible versions of people's selves and lives (Lieblich et al., 1998). People make meaning of the social world around them; they construct their identities and self-narratives from building blocks available in their common culture, above and beyond their individual experience (Lieblich et al., 1998). By studying and interpreting participants' narratives, the researcher can access not only the individual identity and its systems of meaning but also the teller's culture and social world (Lieblich et al., 1998). Because of its ability to elicit rich, descriptive, personal accounts of events, narrative research is recently gaining popularity in the field of nursing.

A narrative methodology is the most appropriate choice for this study because it can provide insight into the personal experiences of individuals (Jakobsen \& Sorlie, 2010; Kucera, Higgins, \& McMillan, 2008). The fact that it is grounded in stories will allow me to elicit rich data that is based on personal experiences occurring in the care setting. A narrative study can yield important knowledge that is gathered from personal accounts through various data collection techniques including interviewing and visual narratives, in the form of photographs. 


\section{Study Design}

For this study, I recruited two participants through purposeful sampling from an ED of a large metropolitan academic teaching hospital in Ontario, Canada. Purposeful sampling allowed me to hand pick cases that enabled detailed exploration of the research phenomenon (Creswell, 2007). To gather a range of data, I focused on recruiting participants with varying years of nursing experience. According to Benner's (1984) stages of clinical competence, when nurses transition along the experience continuum from novice to expert they develop and establish clinical competencies and knowledge, as well as skill in communication and therapeutic relationships with their patients. In a qualitative study by Chang et al. (2009), the authors investigated the challenges associated with caring for patients with dementia. Participants in this study indicated knowledge and skill in dementia care is a necessity in the provision of quality care. Therefore, a novice nurse with limited skills and understanding of dementia care may provide a different account of what the experiences in caring for individuals with dementia would be like when compared to a more advanced nurse who may have developed enhanced competence in caring for individuals with dementia. In narrative research most sampling types are variations of purposeful sampling (Holloway \& Freshwater, 2007).

The first step in accessing participants was through gaining entrée into the site (Creswell, 2007). Gaining entrée involved first being introduced to the ED manager by a member of the senior management team at the hospital, then seeking permission from the ED manager who permitted my entry into the nursing department. Through discussions with the ED manager, the most effective method for participant engagement was identified. Participants were recruited by two methods. These included departmental e-mail distribution of a recruitment letter by the 
department ward clerk (Appendix C) and a recruitment poster that was posted in the ED staff room (Appendix D) inviting interested nurses to directly contact the principal investigator. Approximately two weeks later, I received a total of two responses from two nurses with diversity in their years of nursing experience who demonstrated an interest in participating; therefore, consideration was given to both participants. Participants for this study included two nurses who worked in a single ED. My first participant Eva has been a nurse for about 15 years and spent over 14 years of that as an ED nurse, while my second participant Beth, a recent nursing graduate, has five years of nursing experience and has always practiced in the ED. Both these participants have been provided with pseudonyms. Because narrative research seeks to collect in-depth information on experiences of individuals, the researcher spends considerable time with as few as one or two participants (Creswell, 2007). The purpose of this study was to gain a rich understanding of the everyday experiences of emergency nurses through their stories. In this study, I elicited and analyzed the stories of two participants in order to provide in depth information related to the study purpose, rather than interviewing several individuals who fulfill the breadth of this topic. In narrative research, the focus is to explore the meanings of stories of a particular group of people with the goal of deeper understandings of a specific phenomenon, as opposed to claiming generalizability of findings (Holloway \& Freshwater, 2007). The quality and richness of information provided is more important than the numbers in the sample of narrative research (Holloway \& Freshwater, 2007). When a homogenous sample is selected as the population for the study, as is the case for this study, a purposefully selected small sample size is suitable to address the research goal (Holloway \& Freshwater, 2007). 


\section{Data Collection}

During the recruitment phase, I met with interested and potential participants to review details of the study including the study purpose, expectations of participation, and privacy and confidentiality. Individuals were provided with time to have all of their questions answered. When individuals indicated an interest to participate, I reviewed the consent form with them (Appendix E). Also, I answered any of their additional questions followed by having them sign the consent form.

Two semi-structured, individual, one-on-one interviews using open-ended questions were used for each participant (Appendix F/G). Interviews were scheduled with participants 2-3 weeks apart at the participant's convenience. The semi-structured interview guide was also bolstered with an emergent approach in which prompting questions were asked for clarification and elaboration allowing for an in-depth understanding of their experiences (Creswell, 2007) According to Creswell, open-ended interviews are an effective data collection method because it allows for participants to lead the discussion and researchers to explore and ask clarifying questions.

In my study, I used a creative method of data collection that included visual narratives, in the form of photographs taken of metaphorical objects, which complement interview data and provide access to the deeper meaning of the participants' experiences with the phenomenon. Visual narrative research is a reflective, active human process in which researchers and participants explore and make meaning of experiences through photography (Clandinin, 2007). Sources of narrative data can be enhanced by asking participants to take photographs that can help them express details that they might not include in face-to-face interviews (Duffy, 2007). In 
chapter one I displayed a photo which I combined with my story to represent and enhance the meaning of my experiences.

Participants were provided with a disposable camera and an instruction sheet (Appendix H) during the first interview. They were asked to take photographs of objects or images, excluding that of patients, their families, nursing colleagues or other people. Also, they were asked to not take pictures on hospital property. These images were of nature or inanimate objects that reflected their experiences with respect to caring for older adults living with dementia who present to the ED. Two weeks after the first interview, participants met with me for 5-10 minutes in order to return the disposable camera with photos taken (Appendix I), so that I could develop and print them before the second interview. At the second interview, participants had an opportunity to talk and tell stories about their photographs.

The location of the interviews was chosen by participants with preference given to a quiet, private location, outside of the workplace environment. Interviews were audio-taped and transcribed verbatim by the principal investigator. Field notes were also collected during all interviews. I recorded my thoughts related to my observations of participant's non-verbal and verbal language, the nature of the physical setting, my thoughts, feelings, and assumptions to interview questions and participant's responses, and I also recorded beginning interpretation of interview data. These notes were important during data analysis and also allowed me to be reflexive and open about my influence during different stages of the research process. Interviews lasted approximately 60 minutes; consideration was given to the length of interviews conducted. Lengthy interviews can be tiring for participants therefore length was gauged according to the needs of each participant (Holloway \& Freshwater, 2007). 


\section{Data Analysis}

In narrative approaches, the method of data analysis should be based on the study purpose (Clandinin, 2007; Lieblich et al., 1998). In this study, the selected approach to data analysis was based on the goal of the research question: to narrate, specifically, the experiences of emergency department nurses caring for patients with dementia. Therefore, an analysis for reading, analyzing, and interpreting narrative data in this study was conducted using CategoricalContent and Categorical-Form analysis as proposed by Lieblich et al.

A categorical-content approach is similar to classic thematic content analysis, which involves an analytical examination of the story or specifically selected sections that are relevant to the research question in order to identify themes and patterns among the themes (Burnard, 1991). This categorical approach is in contrast to the holistic approach, which concentrates on analysis of the participant's entire life story (Lieblich et al., 1998). I was not focusing on the participants' entire life story, but only a portion related to their experiences in the ED with patients who have dementia. In categorical-content analysis the researcher analyzes interview data concentrating on what happened, why, who participated in the event, as shared by the teller of the story (Lieblich et al., 1998). Additionally, the categorical-form approach examines the stated feelings evoked in the storyteller as a result of the experiences described in the story, evidenced in the language used to narrate the story (Lieblich et al., 1998). The researcher also pays attention to the style of the narrative, and the choice of metaphors or language used by the narrator, allowing for a deeper analysis of the participants’ story (Lieblich et al., 1998). Photographs and direct quotes have been used in conjunction with the main content to depict, illustrate and enrich parts of the story and to engage the reader in the deeper meaning of the participants’ experiences. 


\section{Categorical-content analysis.}

I analyzed participant's entire interview transcripts obtained from both interviews using categorical-content analysis. I adhered to the steps of categorical-content analysis of interview data as proposed by Lieblich et al. (1998) following these steps:

1. Participant's stories from both interviews were collected, read, and re-read separately to obtain an overall understanding of the experience shared. When handling narrative material I alluded to the importance of dialogical listening, specifically to three voices: 1) the voice of the narrator provided in audio-tape and text, 2) the theoretical framework, specifically CST, which provides the tools of interpretation for this study, and 3) being reflexive while reading and interpreting data. In this process the researcher enters an interactive dialogue with the data to find meaning.

2. A selection of texts was made from the entire interview data of each participant based on its relevance to the research question. For example, any utterances or groups of sentences related to how emergency nurses narrate their experiences of caring for patients with an underlying diagnosis of dementia were extracted from all of the transcripts.

3. Essentially, the selected sections of the text were withdrawn from the total transcripts. Then, several categories were constructed from the text of both interviews.

4. To refine the many categories that emerged, major content categories were defined and named after extensive reading and analysis of interview data. This step involved reading the extracted subtext and associated categories as openly as possible. With consensus from the thesis committee we defined four major content categories or broad narrative ideas. Finally, texts determined from step three were sorted into relevant narrative ideas 
5. Conclusions were drawn from the contents collected in each narrative idea and used descriptively to formulate the findings.

6. The same pattern of analysis was undertaken when performing a categorical-content analysis on text related to context. Two major narrative ideas emerged in this analysis.

\section{Categorical-form analysis.}

The purpose of categorical-form analysis is to elicit a better understanding of the story by focusing on the stylistic and linguistic characteristics of the narrator that might not have been apparent from content analysis alone (Lieblich et al., 1998). Form analysis requires the researcher to engage in examination of the deep meaning of a text (Lieblich et al., 1998). Categorical-form analysis requires detailed transcription of narrative form elements including the use of adverbials, denotations of time and place, passive and active forms of verbs, intensifier words, intonations, length of silences, repetitions, as well as paralinguistics (Lieblich et al., 1998).

I carried out form analysis on interview data, with particular emphasis on the cognitive and emotional episodes of narratives that were related directly to the participants' account of a significant experience with dementia. Conclusions about the narratives were drawn by four methods of analysis. Leiblich et al. (1998) utilized a cognitive analysis framework, which looks deeply at participant's narratives with a psychological lens attempting to reveal, specifically, faulty thinking patterns. My goal of conducting a cognitive analysis is to learn about the narrator's insight, reasoning, and judgment as it relates the specific words and phrases they use in their stories and not necessarily the inadequacy, inability or irrationality in their thinking per se. Therefore, I first analyzed the cognitive elements of language used, which was guided by the Alzheimer Society Person-Centred Language (2012) and the Alzheimer Society Shifting Focus 
(2013): a guide to understanding unpredictable dementia behaviour, to analyze the cognitive elements of participant's narratives. Second, using Leiblich et al.'s (1998) approach, I analyzed the linguistic features in the content of interview data. Third, the paralinguistic data collected during interviews, which I had documented in my field notes during both interviews. In a fourth method of analysis, I accessed a list of formal elements of language and mental processes provided by Leiblich et al. (1998) to further guide the analysis of emotionally charged narrative.

\section{Evaluation of Rigor in Narrative research}

In this study, rigor was addressed using Lieblich et al.’s (1998) criteria for the evaluation of narrative studies: width, coherence, insightfulness, and parsimony. One of the ways rigor is addressed in other qualitative research designs is through establishing the trustworthiness of qualitative data through enhancing credibility, dependability, confirmability, and transferability (Streubert Speziale \& Carpenter, 2007) However, these four criteria for evaluating rigor are general and not specific to any particular methodology whereas, Lieblich et al's criteria are specific to narrative components related to form and content of stories.

The first criterion is width or comprehensiveness of evidence (Lieblich et al., 1998). This dimension is concerned with the quality of data collection and interpretation of findings, as well as the use of numerous participant quotations to report in study findings (Lieblich et al., 1998). In this study, I addressed width by submitting interview data to multiple levels of data analysis from narrow categories and themes to more abstract major narrative ideas. Furthermore, subjecting interview data to both categorical-content and form analysis allowed me to provide in depth interpretation of findings and explore the possibility of alternative meanings of data. In addition, since the way people see the world is closely connected with their choice of words, to separate people from their language is to remove an important part of their reality (Blauner, 
1987), therefore direct quotations have been used throughout the research report to depict participants' thoughts. Finally, I achieved width by taking findings, interpretations and emergent themes back to study participants to ensure their accuracy in reflecting their experiences.

In this study, attention was paid to coherence, that is, how different parts of data interpretation create a complete and meaningful picture (Lieblich et al., 1998). I have accomplished this through analysis of form and content, as well as visual illustrations to create a meaningful picture of the narratives. Also, I have treated participants' stories individually as a whole, as well as in the context of each other. I then considered stories in the context of existing literature and how they fit with current literature; as such, I maintained a commitment to the coherence of the findings (Leiblich et al., 1998)

Insightfulness is concerned with "originality in the presentation of the story and its analysis” (Lieblich et al., 1998, p. 173). The intent of this study was to provide the reader with insight into the lives of emergency nurses and their experiences providing care for patients with dementia. This was provided, with originality, through photographs with participants' explanations, stories, and a critical discussion of narratives to increase reader's comprehension of the research topic. Insightfulness was also achieved by providing a vivid experiential account of the research phenomenon through my own story in chapter one as well as participants' stories.

Parsimony is concerned with the quality of the written story and its analysis (Lieblich et al., 1998). In other words, how representative and reflective the interpreted material is to that of the participant's voice. In this study, all interview data collected was audiotaped to ensure that important information was not unintentionally omitted. Also, during the second interview there was opportunity to ask clarification questions. This allowed me to understand meanings and interpretations of words and statements made by the participants, to ensure that data gathered 
accurately reflected participants' views. In this study, field notes were used during interviews. This allowed me to be reflexive by recording and reflecting upon my observations, thoughts, feelings and emotions during both data collection and analysis of transcripts. For narrative data to be considered trustworthy the researcher will, through note keeping, uncover their own interests and background, and how these influenced the research strategies and procedures (Holloway \& Freshwater, 2007). This was particularly important in this study as I work in a setting similar to my participants, and being aware of my own stance on issues was imperative.

\section{Ethical Considerations}

During the implementation of this study I adhered to the principles concerning ethical research conduct such as confidentiality, informed consent, protection of participants, and sensitive topics. First, I received ethics approval from both the Ryerson University Research Ethics Board and the hospital Ethics Board. According to Speziale and Carpenter (2007), small sample size and thick descriptions can make maintaining confidentiality difficult in qualitative studies. Confidentiality and anonymity were addressed by applying a pseudonym to each participant and anonymizing the institution where the participants were employed (Creswell, 2007). Informed consent fulfilled the ethical principles of autonomy, purpose of study, confidentiality, and risks and benefits of participating in the study.

There are three potential ethical concerns that I identified as most significant for this study. First, there was potential for a close relationship to evolve between the participant and me as the researcher during interviews and thus, participants could have confused my role as a researcher with that of a counselor. In this case, I consciously maintained focus on the topic and guided the interview accordingly (Speziale \& Carpenter, 2007). The option of being referred to appropriate counseling services provided by their institution was also considered. Second, I realized that 
participants could become emotionally distressed while sharing their personal experiences. I addressed this by being prepared to advise participants that they could end the interview sessions at any time (Speziale \& Carpenter, 2007), reschedule, or take a break, and start again. Finally, because of the nature of narrative research there is a possibility that participants may be identified by those who know them in the study's final report. This has been addressed by omitting any recognizable identifiable demographic information, thus assuring participants that their participation will be confidential (Holloway \& Freshwater, 2007).

In the next two chapters, I present the findings from a categorical content and form analysis of interviews with participants Eva and Beth, respectively. I begin each narrative by briefly illustrating an image of the participant and providing a description of the setting in which their interviews were conducted. I discuss each narrative separately, and then conclude this chapter by outlining the similarities and distinctions evident between their stories. 
CHAPTER 4

FINDINGS: EVA 


\section{Eva}

I introduce you to Eva, an experienced ED Registered Nurse, in her late 30’s. After making telephone arrangements with Eva, we first met in the hospitals front lobby. As Eva walked through the door all I could notice was her smile. Without a doubt in my mind I knew it was her. Still, I cautiously approached her and introduced myself. She wore blue jeans, a light coloured t-shirt and carried a small purse over her shoulder. Eva had a confident and easy-going demeanor about her. She was dressed comfortably and did not appear nervous; she walked briskly with her head erect, smiling.

Being a visitor of the hospital I requested that Eva lead us to a quiet and private location to have our interview. Eva and I walked side by side through the hospitals large front lobby, passing the information desk, gift shop and into a long narrow hallway while engaging in some general conversations about the hospital. On route, Eva stopped to talk to a familiar patient. Not wanting to intrude on their conversation, I somewhat distanced myself from them until they were finished. After a few moments and steps away we went up a set of elevators to an area that overlooked outside. Eva said traffic was light in that corridor and suggested we sit on the couch that was close to the elevators. Eva placed her cell phone and purse on the small table next to us, and I placed my note pad on my lap and tape-recorder on the armrest of the couch. We reviewed the consent form in detail at this time. The nature of our first encounter was relaxed. Prior to beginning the interview, Eva voluntarily shared personal stories about her nursing experience and her desire to pursue a master's degree. This helped to ease tension and build rapport. During the interview it was evident that Eva was very astute. After each question Eva immediately provided answers, as though she knew in advance what I would ask. Her responses to questions 
were direct and did not have a great deal of detail, however provided enough information from which to uncover meaning.

\section{Categorical Content Analysis}

During categorical content analysis, it is sometimes important to provide contextual factors, which are interview material that are outside of the selected subtext, for two reasons: 1) it provides a more holistic representation of the participant, and 2) it supports and validates interpretation of the study results (Leiblich et al., 1998). Therefore, content from the complete interview was analyzed and subsequently two narrative ideas that were not directly related to the research question but provided context emerged. These narrative ideas that are contextual were named as: 1) In the beginning, and 2) Inclination for Acute Care. Through ongoing dialogue with the interview data that related directly with the experience of caring for persons with dementia, four narrative ideas that were dementia-specific emerged: 1) Dementia: experiential knowledge;

2) Person-centred care: the impossible; 3) Emotional labour of work; 4) Support and appreciation.

\section{Narrative Ideas - Contextual}

When I began examining Eva's transcripts my initial thoughts were that I would place emphasis on analysing and interpreting her experiences as an ED nurse caring for patients with dementia. After carefully listening to and reading Eva's transcripts over and over I discovered an important pattern woven throughout the stories she shared with me. I realized that disregarding Eva's stories that preceded her nursing career would likely dismiss important segments of her life events that otherwise depict who she is as an individual. Collecting and analyzing this early career information contributed to my understanding of Eva's professional identity as a dementia caregiver. After thoughtful and careful interpretive analysis of Eva’s interview transcripts I 
recognized that her past had a significant impact on most of her experiences as a nurse; in particular, it influenced her viewpoint of and interactions with people with dementia. Therefore, I first discuss the contextual narrative ideas that emerged beyond the main research question.

\section{In The Beginning}

Prior to her nursing career, Eva worked in a hospital as a service worker, while working she was also studying to complete a diploma in a field other than nursing. When I asked Eva to tell me more about why she chose to be a nurse, she recounted a significant story of a time when she worked as a service worker to patients on a hospital ward:

I went into room 312, the call bell had been going off ... there was an elderly woman in bed number two and a young woman in bed number one ... the elderly woman was concerned ... she was calling for a nurse but no one was coming, [she asked me] if I was a nurse ... and I didn’t know any signs of a heart attack or anything ... turned out no nurse had come and I couldn’t find a nurse. As soon as I got to the stairwell to go downstairs a code blue was called on the young girl, she passed away. I realized at that time I wanted to become a nurse to make a difference.

In this excerpt Eva narrates an event of intense nature and a turning point in her life that influenced her decision to actively change her career path to nursing. Eva’s decision to become a nurse was an intentional and planned endeavor.

Being witness to a dying patient while not having the knowledge, skill and ability to intervene, evoked feelings of incompetence and helplessness in Eva. She stated "when nobody [came] I felt frustrated and sad for [the patient] ... I didn't know anything with what was wrong with the young girl.” She further postulates how the elderly woman in the room may have felt, stating "I could see where the elderly woman was coming from she is trying to help but her 
hands are tied." Eva's recollection of this elderly patient is described as someone who is unable to physically prevent the death of a patient. Without any motive or awareness, Eva describes her own limitations related to this incident, and therefore desires to be a nurse to make a difference between matters of life and death. This excerpt reveals Eva's inclination to work in a capacity where her efforts have tangible, measureable, and positive outcomes. The pivotal moment Eva has narrated indicates the value she attributes to interventions that address health concerns of an acute nature, with the potential to cure a patient. Ultimately, the intervention typology that Eva values relates to highly observable outcome - the restoration of, and saving of a life. This passage represents Eva's history and motives, which are an important consideration throughout the remainder of her narratives.

\section{Inclination for Acute Care}

The narratives collected from Eva consistently represent her desire for acute care nursing, before and during her career as a nurse. While a nursing student, Eva indicated that she was drawn to courses such as pathophysiology and statistics because the objective nature of these courses provided a challenge and mental stimulation. In contrast, Eva refers to nursing courses with dissatisfaction. She reflects on her educational experience in her previous career attributing greater value and appreciation to the academic content learned there when compared to nursing school. For Eva, nursing school was elementary, so much that she never reviewed any content, was heavily occupied with a full-time job and managed to graduate with honors. She stated:

If I was to compare to [my other career] ... it was definitely more challenging, nursing is all foundational stuff ... all the different theorist like Roy and Watson ... nursing [should be] defined by your own definition ... values ... beliefs ... rather than being told how to perform a nursing assessment. Nursing is very subjective, whereas [my other 
career] was definitely more objective. In nursing school [you learn] 2 different types of arthritis, a lot is missed out or cut out ... but [in my other career path] you went into everything in depth. Not many [courses] were challenging [in nursing] ... my books were still in cellophane wrap [and] I graduated at the top of my class.

Eva held a preconceived image of what nursing meant to her. She entered nursing school with a goal to one day save lives, but was disappointed to see the lack of medical knowledge she could attain from a nursing curriculum.

Opportunities to feed her passion for acute care nursing were only realized when Eva was given a rotation in the ED as part of her experience in a clinical setting prior to entering the workforce. It was precisely her experiences in the ED as a student that motivated her to continue on the same path and find work in an ED. She stated: "as soon as I finished my last placement in the emergency department as a nursing student [I was motivated to work in the ED] ... It was the variety of patients, all the different things people present with ... the continuous new learning that is hands-on.” In this narrative, Eva exemplifies her passion for change and challenge. The constant turn-over and high acuity of patients fuels Eva. Eva wants to work in a capacity where she can see and measure, objectively, the impact of her nursing care. However, Eva's expectation from her ED career was not always what she imagined.

Eva started her career with hope that the ED would be similar to what she viewed on hospital television programs or experienced as a student. The realities of the ED slowly revealed itself to Eva, who stated:

I thought it would be more trauma cases, like the TV shows ... all the blood, shocking, [and] asystole ... just the excitement of it ... but a lot of times it's more the clinic 
cases, or the elderly people with CHF or COPD, just presenting to the ED because they're not managing their pills, their bodies have finally given up.

It is apparent by this passage that Eva has little interest in managing chronic diseases such as CHF or COPD. These diseases develop in older age and usually have a slow and steady progression with a predictable course of treatment and outcome, in contrast to what Eva wanted from a nursing career. Eva values problem-solving and critical thinking during nursing care and even the use of technology as she referred to "shocking" and "asystole" being an exciting part of the job. She narrates thought-provoking patient encounters with enthusiasm:

It's challenging if someone comes in with something I don't know about ... I have to look it up ... if I give medication like Fragmin ... it may contradict with their other underlying conditions ... [for example]... if they have platelets lower than $50 \ldots$ I would hold the [Fragmin] ... they all come in with at least 2 or 3 [conditions] ... it's just a pain to dispense 20-30 different medications.

While she enjoys problem-solving around medications and the impact of these on patients in the context of acute syndromes, this does not extend to caring for older individuals with chronic conditions. Dispensing 20-30 medications is a repetitive task for Eva and exemplifies her dislike for managing chronic conditions that may not have an immediate measurable or tangible outcome.

\section{Narrative Ideas - Dementia Specific}

The proceeding narrative ideas are derived from subtext of interview data that related to the research question: How do emergency nurses narrate their experiences of caring for patients with a diagnosis of dementia? 


\section{Dementia: Experiential Knowledge}

I chose to refer to this narrative idea as experiential knowledge because it was knowledge Eva developed over a period of time through her direct interactions with patients who had dementia rather than from theoretical or empirical sources. After careful readings of Eva's interview transcripts I was surprised at the variation between the spoken words and the photographs she had taken to describe her experiences with patients with dementia. The former had a slightly negative connotation during narration, while the latter was illustrated and described more positively.

Prior to being a nurse, Eva was unable to decipher dementia from expected forgetfulness of aging; all symptoms were compartmentalized and described as a natural aging process. She stated: "I didn’t really know a lot about it until I became a nurse, I just thought that everybody who gets old is gonna get forgetful.” Through experience Eva’s knowledge of dementia increased, she began to notice behavioral changes in patients with dementia. She stated:

When I started in nursing. . . I guess around 7 o'clock [pm], that's when you start to notice sundowning ... confusion would set in ... patients would be more agitated and confused, you didn’t need a clock, you would walk into a patient's room and [know]. Eva recognized these changes in the patients with dementia and given her desire to learn she made inquiries and substantiated her knowledge about sundowning through research. In current literature this is better recognized as delirium. She stated "I started to do my own research and ask questions about it.” Eva shared the details of a story about a patient who was brought in to the ED to be treated for an infection, she stated:

Her neighbor brought her in . . . the patient was confused, but normally she wasn't confused, it was just slight dementia going on ... [when the family left] ... the patient 
had to continually be re-oriented ... as the night progressed it was more confusion ... she was asking about breakfast at night ... things just weren’t making sense.

Eva had difficulty articulating what she experienced with this particular patient. She knew that there was an element of confusion, but did not have the knowledge to further understand the physiological or psychological symptoms that this patient presented with. Further discussion with Eva about patients with dementia indicated that in fact these patients may not be confused, rather they may be intentionally displaying these chronic behaviours so to receive attention. Eva stated:

As I was caring for this patient with dementia ... I brought her a bed-pan every 15 minutes ... I was constantly repeating myself ... I hate to say it but it was like an attention-seeking behaviour ... if she genuinely was forgetting then I felt sad for her. This excerpt further reflects Eva's assumptions about dementia behaviours. Eva draws inferences based on repeated events rather than theory or evidence. In the above narration, Eva questions the authenticity of the constant requests from the patient, creating an overall negative experience with a person with dementia. Her description of the person with dementia varies and was impacted by whether a particular experience was positive or negative.

Eva took photographs that she defined as illustrations of her understanding of dementia. Unlike her verbal accounts of dementia experiences, the photographs were taken without any reference to a particular patient. Additionally, her accounts of the photographs generally have a positive tone. Image 2 and 3 are two of the six photographs Eva captured. These were selected and displayed in this section to further describe Eva's understanding of dementia. I chose to display this photograph to exemplify the contradiction evident between Eva's photographs and 
personal experiences with dementia. She stated:

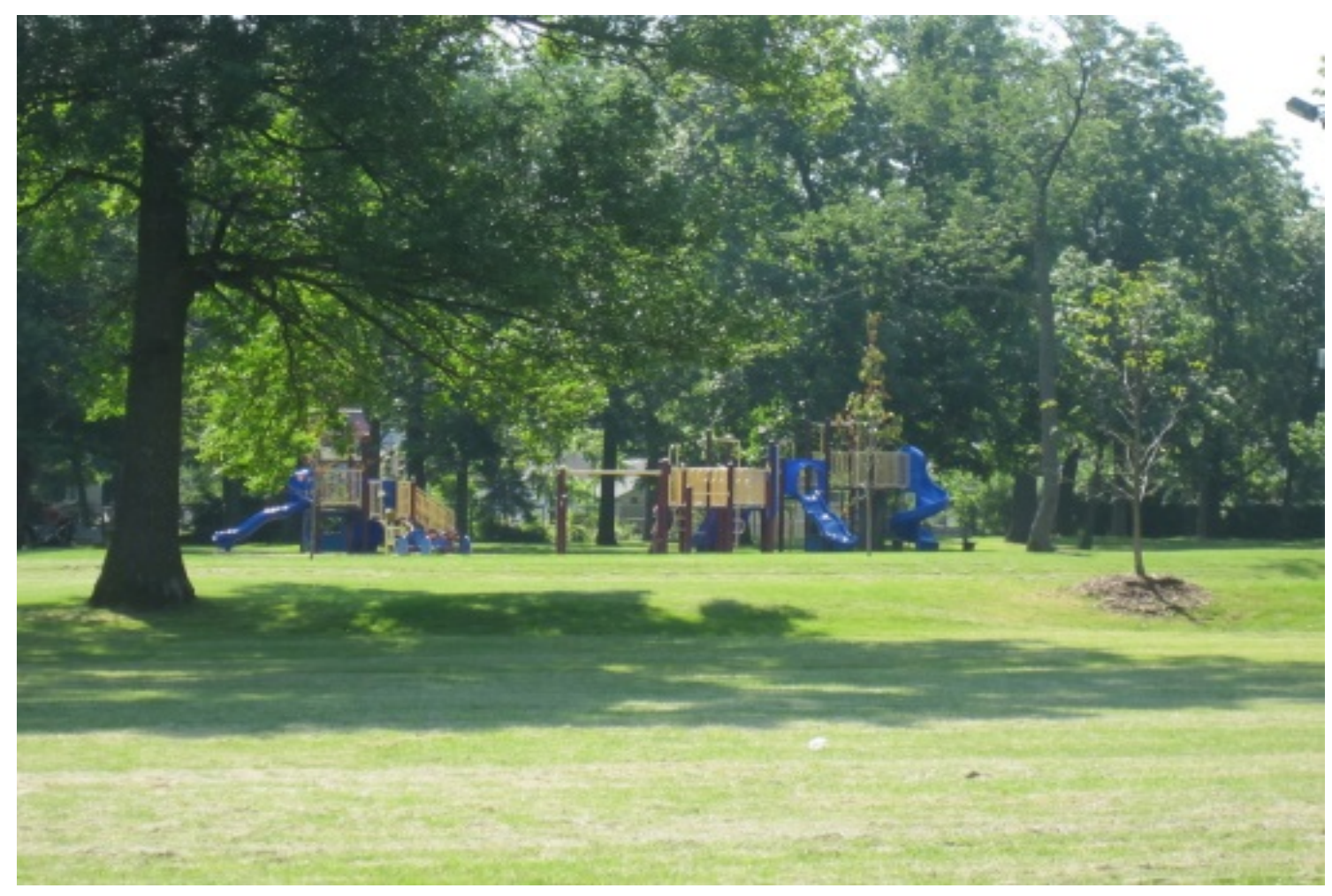

Image 2: Playground

This is a picture of a playground; it makes me think about how the elderly revert back to childhood, when I saw this playground, life just seems so simple, there are no time constraints of anything, and if they're pleasantly confused, it's like the child, they're innocent and peaceful. While taking this photograph I felt safe, fun, peaceful and happy. 


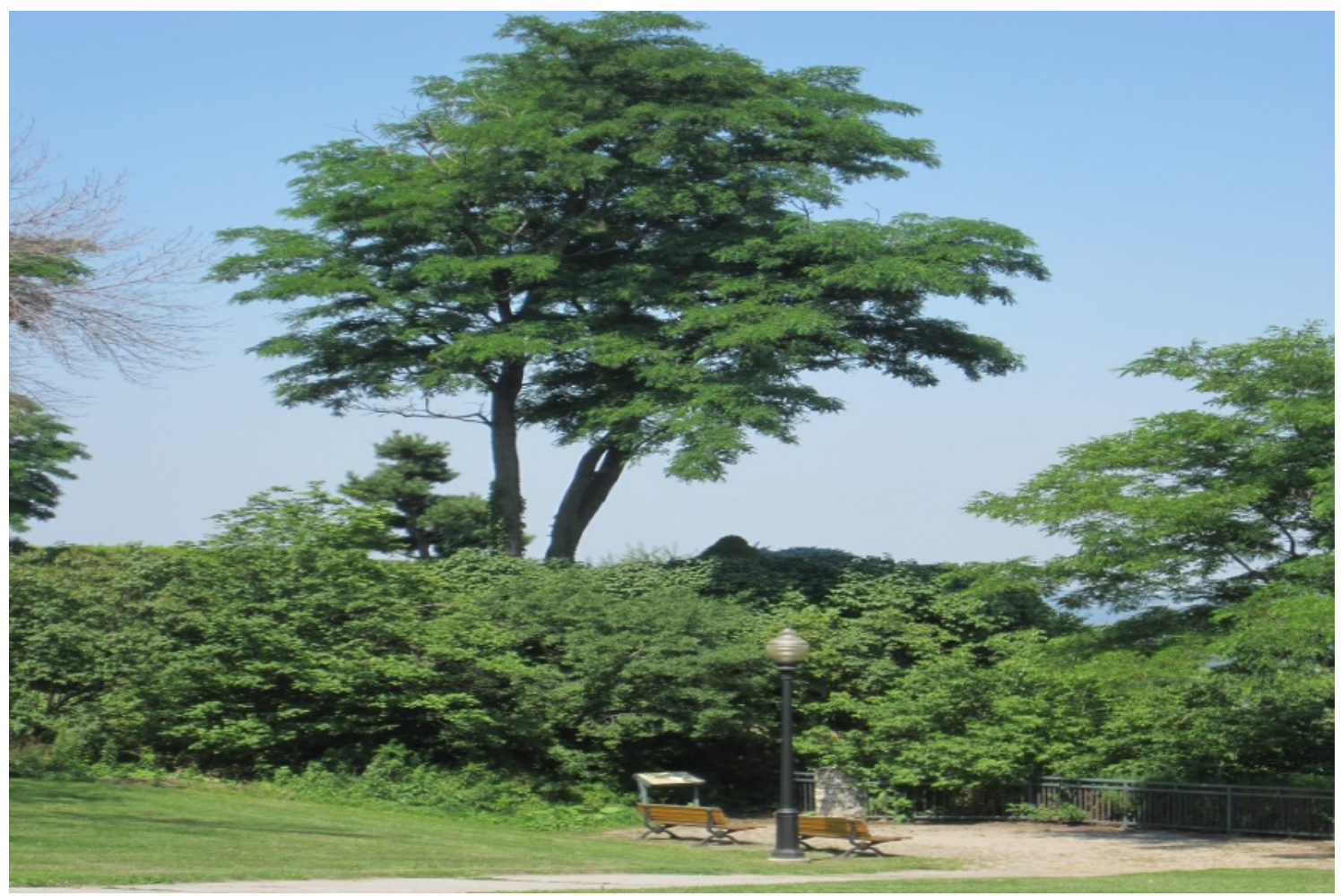

Image 3: A Split Tree

I pass this tree every day, I see this tree as a tree of power and beauty, it stands alone, overlooks the city, it's sort of peaceful, [looking closer at it] it's split into 2 and the one stands up tall I see as being strong, being put together, the leader. But then another branch is kind of bent off so it's sort of to me kind of somebody with early onset dementia, they're still standing tall, but . . . they are becoming more forgetful . . . It's a quality of life that we have to appreciate, a new quality of life ... I find peace in that.

Eva's description of image 1 and 2 is comparatively positive in relation to her personal narratives with patients with dementia. It is fair to say that because the photos were taken outside of a work context and in an appealing location it may have induced more positive emotions in Eva, while allowing her more time to reflect on what dementia means to her. Whereas, when Eva narrates the experience of caring for patients with dementia amidst the hustle and bustle of a busy ED her 
recollection of events and experiences were unpleasant, and her corresponding descriptions reflected this.

In these photographs, Eva speaks to the diversity of patients with dementia; she associated the pleasantly confused patient with dementia as a peaceful person who is innocent. Although Eva captured these images to depict her definition of dementia, it also brings meaning and sense to Eva's experiences with dementia. For Eva, the institutionalized patient with dementia is described as confused, disoriented, and demanding while the person with dementia in the community is pleasant, peaceful, and innocent. When Eva is asked to go out and take photographs of images that reflect her experiences, she sees the humanity of the person with dementia. She had more time to reflect on her experiences, which allowed her to see the many sides of the person with dementia. Therefore, an empathetic shift occurred for Eva who captured what it is like to be a person with patient, rather, than how it affects her day-to-day work life. In contrast, the person with dementia in an institutionalized setting is described negatively. This can be attributed to the fact that the person is in the ED and out of their own element and therefore displays responsive behaviours.

These are contradictory images when considered in light of Eva's narrative story. The majority of Eva's knowledge of dementia is accumulated from her personal experiences with it, and those have been predominantly unfavorable. It is plausible to say that when persons with dementia enter the ED as patients they interfere with Eva's work flow by demanding much of her time and attention. Therefore, her perception of the patient evoked feelings of guilt and the language of her narratives became more negative. In contrast, a person with dementia in the community who has not required physical and emotional attention from Eva is viewed as a real person and described as pure by Eva. 


\section{Person-Centred Care: The Impossible}

Eva's narratives express a dissonance that she experiences between the care she believes patients with dementia deserve and the lack of resources that are available to provide that care. Eva recounts the details of an emotionally charged event:

There was an elderly confused patient; he shared a room with a younger patient. The young guy must have been stuck in bed, he couldn't physically move; and the elderly man was very confused and I guess needed to go to the bathroom so he went to the younger guy and urinated on him. The young patient was upset and rang the call bell. The male nurse looking after them went in the room. Here is this old man standing there naked standing at the other patients bed. The nurse shouted at the old man . . . [that's when] I went over because I heard the shouting. I witnessed [the nurse] basically grab this elderly man and throw him into bed, and started shaking him, screaming at him saying stop it right now or I'll tie you down, he hit him up with Haldol and tied him up. I knew the behaviour was wrong, being a new nurse I was afraid to [get involved], I turned a blind eye to it.

The timing of this event occurred in the beginning of Eva's career. Those same feelings of helplessness and incompetence she experienced as a service worker resurfaced. Eva became a nurse to make a difference, to have an impact and to save lives. This time Eva felt that she did not hold enough power or authority because of her lack of nursing experience to intervene and protect this patient from being restrained and sedated. As a novice nurse, Eva experienced a discord between what she felt was the right thing to do and her ultimate actions. To possibly alleviate the guilt she experienced with her decision not to intervene, Eva attempted to justify the nurse’s actions, stating: "the patient was far away from the nursing station . . . he did need to be 
babysat, he wasn't safe to be on his own." What is implied here is that sometimes it is necessary to carry out actions that may limit the patient's movement that are in the patient's best interest and safety. Eva's attempt to resolve the discord requires her to mobilize all of her resources that are in her opinion more person-centred, to manage the patient with behavioural symptoms of dementia.

With the limited resources and context of the ED, Eva provides an alternative approach to managing older patients with dementia. She stated:

My approach would be to take a Geri-chair, put it in front of the nursing station, and he would have been way calmer, [even] slept in the chair. I had a patient once who was pleasantly confused, I'd always get her up to the wheelchair and go racing down the hall, I gave her a pen and paper and she sat at the nursing station with me while I charted, she wrote a letter to her sister, I kept her occupied, I mean, I made the time, on a medicine floor you have that time, but in the ED you don't have the time to do that stuff, instead you move them in the hallway where they are safe, give them sheets to tie ... that will keep them occupied. You give them a project based on their [behaviour], so if they're agitated you give them something to pick at.

Eva attempts to provide the most person-centred care approach within the constraining environment of the ED. She does not have access to nursing aid staff or trained volunteers who could spend considerable time with the patient or geriatric emergency nursing staff who could potentially offer her their expertise and assistance in dementia care. Although not all of her experiences have been positive, Eva describes some creative methods she has implemented. They have not always resulted in a positive outcome but have proved to be beneficial for her when she is confronted with managing her workload and time. She stated: 
Keeping the patient occupied has helped me with my workload ... gives you more time, you don't have to be there as often, but sometimes [patients don’t respond well], I had [towels] thrown back at me and a couple times I've been told to F-off.

She describes what some of her colleagues do as cruel and inhumane, and when given a choice, her methods to manage the behaviours of dementia are preferred. She stated:

Tying them up or medicating them is inhumane ... they should be treated with dignity and respect ... I think sadly it's like putting them on leashes ... it's like we just tie them up to know they are there, we can come back later when it is good for us, I think it should be illegal, because the person's respect and dignity is compromised, they are not allowed to be their own unique individual ... you're basically treating them like an animal, tying them up, shutting them up ... like they're not a person, they're an object.

Eva's narratives continue to suggest that there is a lack of time and knowledge among nurses and when given autonomy, ED nurses will utilize restraints as a first line of defense to manage the responsive behaviours of dementia. This also suggests that dominant discourse of acute medical treatment and physical care is evident in nursing practice. In the interest of time, the care of patients becomes less than ideal. Eva recognizes this and challenges typical ED practices and care of persons with dementia.

\section{Emotional Labour of Work}

The narrative idea of emotional labour of work exposes the many challenges Eva has experienced when caring for patients with dementia. Eva shared with me a detailed account of her experiences while caring for a particular patient with dementia and the implications this had for her and her patient. Eva narrated: 
I recall this lady; she was in her 80's, white hair, plump, very soft spoken. She had dementia ... she was very confused ... and at times appreciative ... she kept asking where she was ... I recall she had an underlying lung cancer and came in feeling more short of breath ... she was brought in by her neighbor who lived in her apartment building; she was a young girl ... the patients daughter couldn't be there. The patient was confused ... especially after the family left ... she needed bed pan and after bed pan and required constant re-orientation, it was challenging in terms of time and energy having to continually provide emotional support to her and settle her. She kept asking, where am I. Why am I not in my apartment? Why am I here? I kept repeating the same thing over and over and over again. It can be frustrating and sad too, the amount of attention they require is frustrating when you have so many other things to do. As the night progressed she was even more confused...she tried to climb out. . . working her way to the ledge of the bed. . . at times she was combative... she would take her fists and just start waving and shouting don't touch me, get away from me... I would settle her back in then things started to get better.

Although Eva is challenged by the emotional and physical investment required by this patient she provided the type of nursing care that met her standards. The repetitive nature of the patient's demands was interfering with Eva’s competing work priorities. For Eva, the factors that contribute to the emotional labour of work are those which suppress and undermine her true feelings and frustrations in order to maintain a higher quality of patient care. She stated: "I feel sort of like a slave, sometimes angry, I can't say what I'm thinking." Using the word slave to describe this experience may suggest that the nature of nursing work in the ED is demanding and the additional attention this patient required was an overwhelming experience for Eva. 
Frustration occurred when Eva was pressured to manage the tensions between the patient needs, her true feelings, and competing nursing responsibilities in the current culture of the ED.

To expand on these experiences, Eva captured Image 4 and 5 that symbolize the challenges associated with wanting to provide ideal nursing care in the midst of limitations.

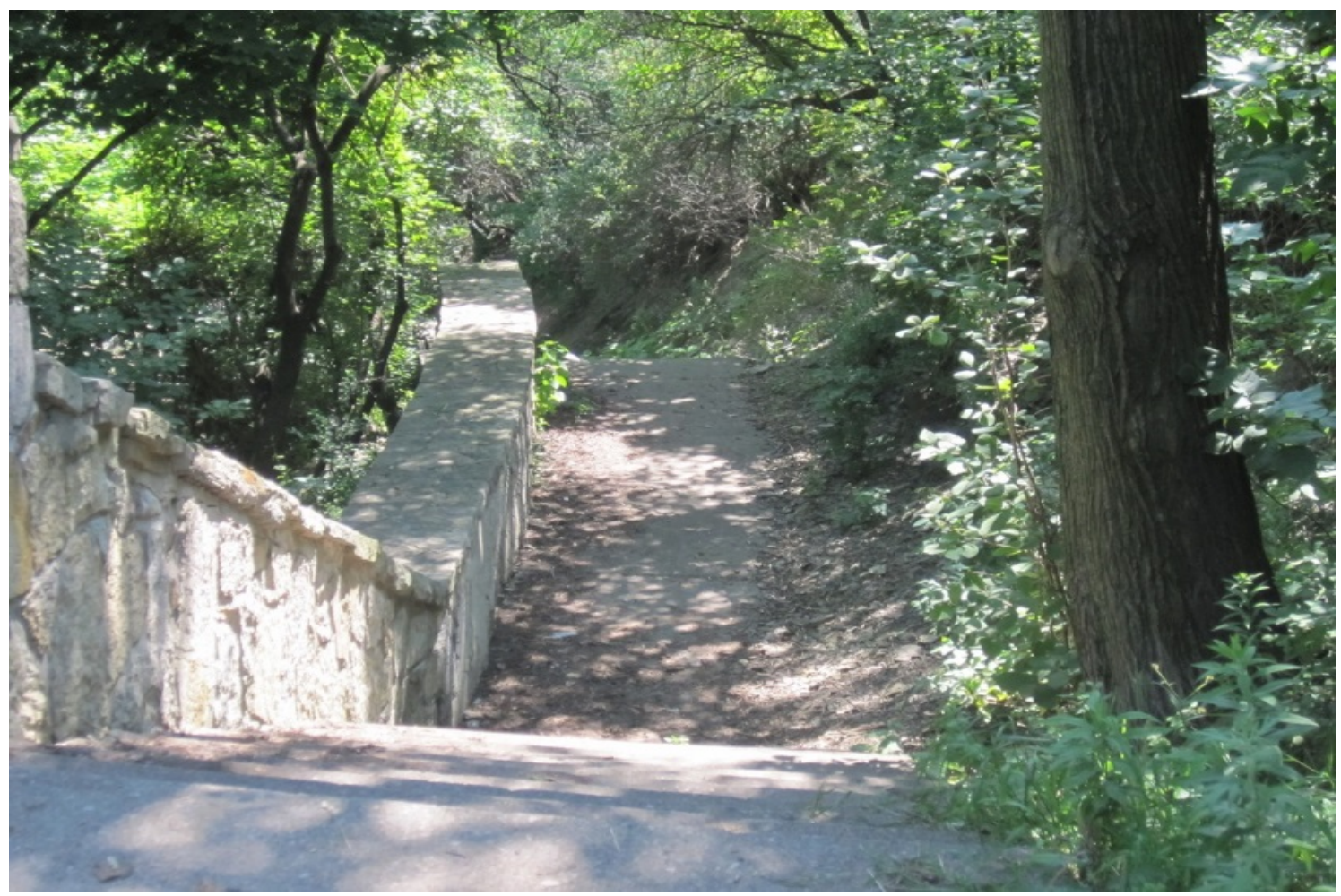

Image 4: A Lonely Path

I look at this picture like a dark hole, I think of how lonely and isolated it must feel as a patient with dementia . . . they are alone going down their own path, nobody seems to understand them . . . it is my feelings too, if they are completely at a state of gone, nothing there, no orientation, it's frustrating, you know you keep trying, and trying and trying but I don't get anywhere. As a nurse sometimes I feel I am on that path. It doesn't matter what I do or say nothing is going to change it.

Image 4 encapsulates the many emotions Eva has experienced. As noted, Eva strives to acquire tangible and positive outcomes during patient care. In emergency nursing there are objective 
means of measuring the effect of nursing care on patient outcomes through assessment of vital signs or symptoms improvement. Positive responses in patients with dementia may not be achieved as simply or measured as objectively as other physical symptoms she has grown accustomed to monitoring. This perpetual state of uncertainty of outcome has resulted in a feeling of incompetency and frustration for Eva. Furthermore, adding to the emotional labour of work is the lack of knowledge available to Eva as an ED nurse to correctly assess and address the needs of the patient with dementia which makes her efforts ineffective.

Image 5 below also depicts the emotional aspects of work Eva experiences.

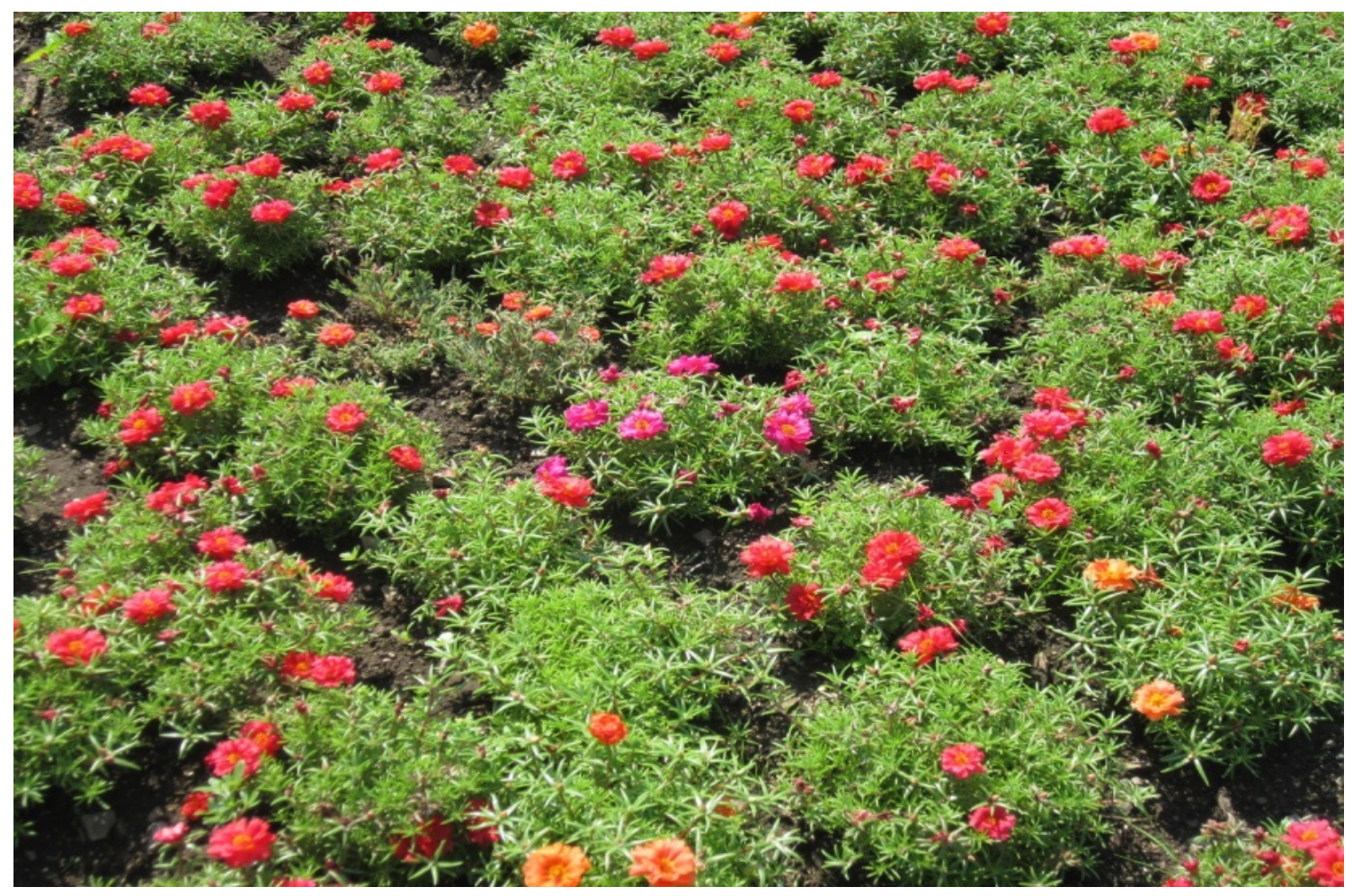

Image 5: The Pink Stands Alone

I saw all these flowers, there were red ones, orange ones, and out of nowhere this pink one. All the other flowers are nursing staff, family members and everybody else, that pink one is the patient with dementia ... despite all of us making one big flower garden, that patient with dementia still sits alone. It is isolating [for everyone] . . . what is it 
really the pink needs, the pink can't really indicate what they need, so there is a level of frustration outside of the pink ... you offer more care . . . it is sad . . . cause you don't understand them.

Eva finds herself struggling to meet the needs of the patient while simultaneously losing the motivation because she is not seeing the positive impact of her efforts. In this image Eva also illustrates that it takes a group of people to manage a patient with dementia. Even with the combined efforts of various individuals, the patient with dementia may not always respond positively to treatment. Symbolically, this patient is different from every other patient; they are alone in the middle. Together these images represent the incongruence between the care Eva wants to provide her patients with dementia and her available resources. In the ED she does not have a "family of red and orange flowers" who help her provide person-centred care. Eva also finds herself alone in giving care, thus she continuously feels frustrated because she went into nursing to make a tangible difference. Caring for patients with dementia has often proven to be monotonous and unrewarding. She stated: "at any given time [their mood can fluctuate], they can be pleasant then suddenly confused, mean, and agitated, and you're like what the heck is going on, it challenges you as a nurse.” As reflected in Eva’s narrative the emotional labour of her work is the difficulties she endures while trying to maintain professionalism and enthusiasm despite her considerable efforts and the lack of desirable patient outcomes.

\section{Support \& Appreciation}

Support from colleagues and family caregivers was a recurrent theme throughout Eva's narratives. Many of the tribulations Eva has narrated are followed with suggestions of how experiences could have improved if she was appreciated and supported. Eva's experiences 
portray the value in simple acts of gratitude to mitigate some of the challenges in the provision of care to patients with dementia. She stated:

I was treated like a slave, [this patient] was very demanding . . . just every time I had to go and bring the bed pan ... she pressed her call bell ... I must have had her on the bed pan 8 times ... if every [nurse] was like me, you just keep giving . . . sometimes you can go out of your way and do everything for somebody, but still it is never enough . . . you ask yourself why am in this profession ... you just don't feel appreciated ... The next day, I did get feedback from the social worker that the patient said I was very helpful ... it was nice to be appreciated . . . because at the end of that shift I was burned-out and frustrated ... it sort of justifies that it was worth it.

This narrative denotes Eva's need for recognition and praise. When her hard work is acknowledged it provides assurance that her efforts are worthy. As a student Eva's motivation to succeed was rewarded by being the top student. As a nurse Eva wants to be recognized for her dedication to her patients; she wants to know that she has made a positive difference in the patient's life, thus mitigating any challenges she withstands.

A strong support system from her colleagues and family caregivers is equally important for Eva. She stated: “. . . You can have 3 patients that are demanding . . . or even their family members can be demanding . . . there is a lack of team work and support from colleagues.” As Eva noted in the photograph of the flowers, it takes more than one person to manage the person with dementia, and even then it is not guaranteed that that would suffice. She stated: “... I have even used [ancillary staff] as a babysitter, [I say] if they crawl out of bed or do anything, to basically call me.” At times the demands can be overwhelming enough to enlist non-regulated health professionals as an extra pair of eyes to maintain the patient’s safety. 
Eva also described the improvement needed in the structural environment of the ED to reduce some of the barriers to care. When reflecting on her experience with the patient with dementia, Eva stated: "I would have wanted to provide more care, but time [was limited], I would have wanted to move her out of that room and closer to the nursing station, so the location and environment [had its own] challenges.” This narrative speaks to the importance of the relative distance of patients to a safe location such as the nursing station, where they could be visible. When patients are not in close proximity, Eva has to spend additional time, which is already limited, to frequently ensure they are safe. Eva does not explicate whether she had the autonomy to make those changes on that shift or not. Moreover, as part of the environmental changes, Eva suggests that keeping her patient oriented was an important role she had that shift. She narrates: "I think having a clock in her room would definitely help, that might have kept with keeping her oriented.” Being provided with a simple piece of equipment such as a clock could prevent the patient's disorientation and thereby promote the patients' independence while reducing her reliance on Eva.

A lack of practical and emotional support from family caregivers of patients with dementia was a key issue expressed by Eva throughout her narrated experience. As she recounted the difficult experience with her patient with dementia a portion of it was related to the patient's family caregiver. In describing family supports Eva stated:

The patient was with this neighbor ... I just thought it was strange that the daughter wasn't there ... when the daughter did come [hours later], she was very abrupt and impatient ... and [after a short time] the daughter left ... she said she had to work in the morning and just up and left. While the daughter was there the patient was constantly struggling to get up ... working her way to the edge of the bed ... and the family would 
just rely on me to turn her and get her back into bed ... the patient was more combative while the daughter was there ... at one point the [daughter and neighbor] stepped out of the room and said to me it's your job, you go and take care of her ... when family members are there you want to rely on them to help you.

The lack of support from family caregivers exacerbated the burden of providing care for this patient with dementia. For Eva, there is no simple solution; she strives to provide care without relying on chemical sedation or physical restraints. Therefore, she relies on her colleagues and family caregivers to help mitigate some of the challenges narrated in caring for the patient with dementia. In the next section I have conducted a categorical-form analysis of Eva's narratives.

\section{Categorical-Form Analysis}

In keeping with the methodological components of Lieblich et al. (1998), instead of purely reporting content as in some other qualitative research traditions, I have provided a higher level in depth interpretive analysis that is grounded in the research data. I have used the Alzheimer Society Person-Centred Language (2012) and the Alzheimer Society Shifting Focus (2013): a guide to understanding unpredictable dementia behaviour to analyze the cognitive elements of Eva’s narratives. Following a cognitive analysis, I looked specifically at the emotional content of Eva's narrative account of her patient with dementia including the linguistics and paralinguistics language, as well as formal elements of speech.

\section{Cognitive Analysis}

In order to preserve the dignity of the person with dementia, appropriate person-centred words and phrases should be used to describe behaviours of dementia (Alzheimer's Society, 2012). It is equally important for healthcare professionals to understand responsive behaviour as often intentional for expressing an unmet need (Alzheimer’s Society, 2013). Depending on the 
language used to describe behaviours, it may suggest that the behaviour is a problem of the person and therefore hinder the provision of timely and effective patient care (Alzheimer's Society. 2012)

The following excerpt is from Eva's complete story, relating directly to her experience while caring for a particular patient with dementia. I have analyzed only pertinent segments of the narrative to demonstrate the limited dementia specific knowledge Eva has, as well as the nonperson centred language used to describe the person and the symptoms of dementia. As I reflect on my own story of Ms. Levine, I too struggled to describe or understand my patient’s behaviours and address her needs in a person-centred care manner. Eva stated:

She was in with dementia; she had underlying dementia as well. She was confused, but normally her baseline she wasn't really confused, it was just slight dementia going on ... it became bed pan after bed pan, after water, after ... just many questions ... having to continually re-orient her, it was emotionally challenging as well as time challenging, just continually having to provide emotional support and settle her ... sometimes she would remember, but quite often she would forget, and as the night progressed it was more confusion ... in the beginning she was more combative ... like when the daughter showed up I found her to be more combative and it was just you're not doing this and would take her fist and arms and just start waving them.

In opening her story, Eva stated the patient came into the ED with dementia and increased confusion from her baseline. Based on this brief description, the patient was likely experiencing an acute mental change from baseline, otherwise known as delirium, secondary to some other process, such as an infection or medication reactions. 
Eva's description of the patient's reason for vising the ED reveals she has difficulty to accurately articulate this patient's medical condition. The patient's behaviour is attributed to the symptoms of dementia, and an important medical condition may have not been addressed in a timely manner. Eva narrates that she had to continually re-orient the patient. Again, Eva was not aware that repetition of the behaviour or verbal requests may be due to the patients' inability to express a need and may have warranted further assessment (Alzheimer’s Society, 2013). Instead Eva continued to re-orient the patient, which did not address the patients' needs nor resolve the repetitive behaviour. The patient would sometimes remember but quite often forget and as the night progressed it was more confusion. This statement by Eva is indicative of a restlessness that occurs in people with dementia at approximately 5:00 to 6:00 p.m. There are several potential reasons for this increasing confusion that requires further assessment and specific intervention to decrease the confusion, such as providing reassurance or comfortable and familiar items to the patient (Alzheimer’s Society, 2013). Finally, Eva stated that on arrival the patient was combative; this term to describe the patient's behaviour is not person-centred. Eva does go on to describe the behaviour suggesting and interpreting the behaviour is a reaction to the daughter visiting. In describing the behaviour this would meet the requirements of person-centred language, as well as a person-centred response since Eva attempted to identify a possible trigger to the behaviour. However, using the term combative suggests that the behaviour is a result of a problem with the person (Alzheimer’s Society, 2012).

\section{Emotional Analysis}

There are three methods of analysis that could be carried out to provide insight into the emotional content of the narrative experienced by Eva. In this section I discuss Eva's linguistics, paralinguistics, and formal elements of speech as they pertain to the above narrative. 


\section{Linguistics: the said words.}

In order to illustrate the emotional content of Eva’s experience I have composed a poem to represent, verbatim, the language of her narrative account of what it was like to care for a patient with dementia.

Eva’s Experience with Dementia

I

recall

One lady

With dementia

She was soft spoken

White hair, plump, in her 80s

Old, appreciative, disoriented

Confused, forgetful, combative

It's sad, no family, escorted by a worried neighbor

A daughter

Hours later

Abrupt, inpatient, disconnected

The lady is agitated, swinging her arms at me, yelling

Leave me alone! Get away from me! Get me out of here!

Daughter leaves. Neighbor leaves. I stay. I am challenged. I have no time.

You will have to rely on me

I'll have to support you

I'll have to keep my eyes on you

I'll have to settle you

This poem, which was approved by Eva, speaks to her aversion for having to provide care for this patient. Her words reflect frustration with the patient’s family and neighbor, who have left Eva, alone, to care for a patient who is demanding and confused. It is possible to infer that Eva does not feel supported. She expresses having no choice but to meet the patient's needs while not finding any pleasure in doing so. 


\section{Paralinguistics: the unsaid words.}

To analyze the paralinguistic properties of Eva's aforementioned dementia narrative, I listened to the audiotape and reviewed my field notes paying special attention to expressions, gestures, volume, intonation, vocal quality, and pauses in speech. In examination of Eva's nonverbal communication I note that overall there were few variations in any paralinguistic features of her narrative. Her speech and gestures were usually consistent throughout the interview regardless of the emotional content of her narrative.

Eva’s gestures and expressions during the time she was recounting a difficult experience were surprisingly relaxed and composed. During the narration of this experience Eva and I sat face-to-face, in close proximity to each other. She had good posture, maintained eye contact, her legs were crossed and arms open, leaning slightly forward, mostly smiling and rarely fidgeting. I presumed that her relaxed demeanor was partly due to the timing of the question related to her experience of a patient with dementia. We were well over mid-way through our interview, by then we had built rapport and Eva was likely more comfortable. Additionally, the recollection of distant memories did not evoke physical changes in Eva’s expressions or body language; throughout the entire narration she maintained a consistent demeanor. Although linguistically the poetic description of Eva’s experience was filled with emotional elements, it exposed no emotional changes in Eva’s non-verbal language. The passage of time likely resolved or suppressed any distressing feelings and emotions associated with this event.

An analysis of Eva's speech reveals small but meaningful variation in elements such as intonation, speed, quality, and pauses during reflections of difficult experiences. In general, Eva's speech was modulated and fast, low-pitch and thin, flowed and was mostly free of excessive pauses or mumbles. Any slight deviation from her normal speech was easily 
identifiable during review of the audio-file. For instance, in the sentence ("a young girl in her 20's brought her in, and stayed with her ...”) there was a small break and increase in pitch when Eva says "with her." It appears as if Eva was surprised that this stranger, a young girl, who is only the patient's neighbor, would do such a compassionate thing. This is normally the role of a family member, and this neighbor is carrying out a significant act of kindness, that Eva finds surprising. Furthermore, in this particular utterance about the patient's daughter (“She didn’t even stop to look at her mother ... there was no connection there”), Eva's tone changed. It started as modulated then raised and emphasized the following: "there was no connection", as if she was asking a question rather than making a statement. Eva was likely seeking confirmation and validation that I was listening and understanding this important part of her story she was sharing with me.

In another utterance Eva seems to find some humor in this difficult experience. She stated: "at one point in time, I'm not sure how or what, but 2 and 2 connected and she started using her call bell ... I probably told her 15 times what it is ... ” Once again her tone changed and this time went slightly higher while her voice quality went from sounding thin to adenoidal. Eva was building up the story to ultimately laugh; she briefly vocalized a giggle after stating "she started using her call bell.” This could also be reflective of Eva’s surprise that the patient who has cognitive impairment from dementia can at times understand and follow instructions.

\section{Formal elements.}

The linguistic features reveal the greatest emotional detail about Eva and her experience in caring for this patient with dementia. In following a modified list of elements provided by Lieblich et al. (1998) I reviewed a subtext of the transcript as it related to Eva's experience of 
caring for this patient with dementia. I concentrated on the chronology of events, intensifiers, and repetition of words or utterances to explore the deeper meaning of Eva's experience.

In a brief preface to her story Eva begins by noting the difficulty in recalling an experience with dementia. She stated "I can’t recall . . . I mean there have been so many of them ... " The intensifier "so many" indicates that repetitive exposure to distressing or difficult events normalizes unpleasant emotions and the memory of them is suppressed until recalling them is necessary. Seconds later, Eva begins to recall an experience: "I can sort of recall one ... she had underlying dementia.” After a brief interruption, Eva began to delve in deeper with her experience of what it was like to care for this patient with dementia. Family and family dynamics played a key role in Eva's narrative. Eva's first memory of this patient actually focuses on other characters, namely a neighbor:

It was sad when she first came in . . . I thought it was her daughter . . . but it was her neighbor in the apartment building that she lives in ... [ [the patient] went to the neighbors door, a young girl in her 20’s who brought her to the hospital . . . she stayed with her until her daughter could actually get there

Eva's immediate reaction to the question was to introduce the neighbor and daughter, indicating their relative importance in her memory of the event.

For a brief moment Eva digressed and in a short sentence describes the patient: "she was confused, but normally her baseline she wasn’t really confused . . .” then goes back to discussing the family again "after everybody left it became . . . it was very helpful to have them there." There are three important elements to mention. First, Eva ends her sentence abruptly after saying “it became,” perhaps she was trying to avoid discussing the challenges she experienced when family left the hospital, however she does revisit the sentence and complete it later on in the 
passage. Second, the conversation veered towards talking about the family once again, without really mentioning when the daughter had arrived, Eva was speaking about her exit. The leap in time is indicative of the emotional attachment Eva has to her abandonment by this patient's daughter. Eva then provides some context for the difficulties she experienced once the family left: "after they left it became bed pan after bed pan after water, just many questions like where am I and having to continually reorient her, it was emotionally and time challenging to continually settle her." Eva then breaks the progression of events and reverts back to describe details about the patient's daughter and neighbor: "like I say she was there with this neighbor from like 5:30 to 10:00 at night, still waiting for the daughter to get there ... I thought it was strange that the daughter wasn't there, when she did come she was very abrupt ... ” This is the second time in the passage that Eva digresses from speaking about the patient and focuses on the family dynamics instead. A third time, Eva describes a situation where the patient's behaviours escalate as a result of the daughter: "when the daughter showed up I found her [the patient] to be more combative and would take her fists and start waving them ... to the neighbor and daughter ... it got to a point where they actually had to step out of the room ...” All the changes in the chronology of the story suggest that Eva was emotionally impacted by the family dynamics. They were significant to the emotional experience of this event, for Eva and the patient.

The repetition of words, sentences, or ideas also provides insight into the emotional charge of a narrative (Leiblich et al. 1998). Key words and utterances that reoccur in Eva's specific narrative related to the experience of caring for the patient with dementia passage in order of frequency are: repetition of phrases from the patient's perspective (11), neighbor (10), daughter (7), challenges (3), family (2), combative (2), confused (2), settle her (2). As examined earlier, family involvement in the provision of care for the patient is important to Eva. A 
majority of her experience with this patient was infused with memories of the daughter and neighbor.

Even while Eva described her own personal emotions: "it was sad when she first came in ... with her neighbor [not daughter] ...” this emotional statement was in reference to the patient not being accompanied by a family member, rather than how she felt about caring for this patient. Therefore, this may suggest that Eva may be distancing herself from the experience. Instead of expressing her reactions to the experience, she quotes the patient during emotional moments. For instance, on several occasions she quotes the patient when attempting to describe the difficulties she had that shift: "she was asking ... where am I ... what happened ... why am I here ... don't touch me ... get away from me ... leave me alone ...” The repetitive use of the words challenging, combative and settle her indicate the emotional labour required of Eva during this patient encounter. Overall, the examination of Eva's narrative reveals a congruency between her speech, gestures, expressions and language.

In the next chapter, I once again use both a categorical content and form analysis to present findings from the second participant, Beth. 


\section{CHAPTER 5}

FINDINGS: BETH 


\section{Beth}

Beth is in her mid-20s, and a Registered Nurse in the ED. At Beth’s request, our first face-to-face meeting was at the hospital, in a private office, after regular work hours. I first met Beth in the hospital's cafeteria. It was easy to separate her from a small crowd. She dressed trendy and stylish. She was wearing blue cropped pants with an off-white maxi blouse, carrying a large brand-name purse, and accessorized her outfit rather nicely with a small charm bracelet. I approached Beth and introduced myself, I shook her hand in a friendly manner, and she lightly shook mine back. I sensed a slight discomfort from her handshake, but attributed it to an expected response after meeting someone for the first time. I followed Beth to the office where our interview would be conducted. On our walk to the office I initiated some elevator talk to reduce tension, which generated fewer responses than I anticipated.

In the office, we reviewed the consent form and shortly thereafter began the interview. I would describe Beth as a quiet and cautious individual. Prior to her responses she would take a few moments to collect her thoughts then formulate a response. I often found myself prompting Beth for further details about her experiences. By the second interview, Beth was slightly more conversational, and needed fewer prompts. However, she was still the type of person who carefully weighed her words before she vocalized her thoughts. Beth displayed greater selfexpression through the photography activity. I found her photographs were packed with feelings and experiences, more so than her articulated words. After combining the interview and photography data I was able to extrapolate great meaning from Beth and her experience with patients who have dementia. 


\section{Categorical Content Analysis}

The following content analysis demonstrates the experiences of Beth. My research goal is to share the individual experiences of two nurses; however, after deep engagement with Beth’s transcripts, I found myself continuously arriving at categories of subtext that were similar to Eva’s. It is my belief that because I analyzed Eva’s narrative first, I followed a similar pattern with Beth’s.

However, due to the limited introductory data I retrieved from Beth, I merged the narrative ideas that were contextually emergent from Eva's interview into one category in Beth’s narrative; thus, In the Beginning and Inclination for Acute Care, which provide holistic and contextual perspectives of her experience are merged into one category, namely: Inclination for acute care. Through continuous dialogue with the interview data that related directly to the experience of caring for persons with dementia, four dementia specific narrative ideas emerged: 1) Dementia: experiential knowledge; 2) Person-centred care: the impossible; 3) Emotional labour of work; 4) Support and appreciation.

\section{Narrative Ideas-Contextual}

\section{Inclination for Acute Care}

Beth had minimal exposure to the workforce prior to attending university to study nursing. She worked for her uncle, in a restaurant. Beth began working in a volunteer capacity at various hospitals, before and during nursing school. Through her many volunteer experiences Beth discovered she wanted to venture into a career in healthcare. She decided to become a nurse. While a nursing student Beth was drawn to courses in the sciences, such as bio-chemistry, while the only nursing course she was fond of was research. Beth explains: "[in nursing] I liked the research class ... we did a study with [one of the researchers] ... after the study [we] put 
together all the data and made a presentation.” In this excerpt Beth demonstrated her interest in problem solving and accomplishing tasks that have well defined outcomes. Beth inferred that compiling and making sense of a large quantity of research data appealed to her analytical and practical perceptions of clinical practice. She further demonstrates appreciation for objectivity stating: "[the science courses], I like them because they are black and white, there is a right answer.”

Beth’s assumptions about a nursing career were based on her observations and experiences as a volunteer. Not having had the opportunity to provide direct patient care left her to assume there was minimal physical contact between patients and healthcare providers, especially in providing hygiene care. The volunteer experiences were not sufficient to help Beth determine whether nursing was the right career for her. Beth stated: “I really didn’t know what nursing was, I didn’t know there was so much bedside, such as the hands-on [stuff] . . . like pericare ...” During nursing school Beth wanted to work in the operating room. The operating room would fulfill her need to do work that is hands-on with technology and equipment rather than having to develop interpersonal communication. In the operating room patients are often sedated or unconscious by medication and immediately discharged to a post-operative area of care when their surgery is complete and they are awake. Beth was disappointed to discover that she was not able to receive a practice placement in the operating room but was rather placed in the ED.

The ED was not Beth’s preferred choice; therefore she initially did not see it as aligned with her career goals. Beth appreciated consistency, well-defined outcomes, and being hands-on with technology instead of patients. The ED would require Beth to manage the technological aspect of care but also spend considerable time talking to patients and their families about their care. The ED is not black and white; patient outcomes are unpredictable, which Beth was 
worried about. She had some doubts about her ability to be successful as an ED nurse. The following excerpt demonstrates Beth’s initial perception of the ED:

I had my last placement in the emergency room which I didn't want, I requested the operating room, they're like ok there is none there so they put me in the ER, I was not looking forward to it, cause I thought it was going to be chaos, difficult and crazy, like what you know from watching it on TV ... you're the first person that people [see], I think what if I don't know what to do, you have to figure out what to do, I was just very scared.

Once Beth gained some experience as a student in the ED she had a moment of realization that much of what she expected to find did not actually occur. Most of her fears were reconciled with positive experiences. Beth's affinity for being hands-on was fulfilled in the ED role. She also began to appreciate characteristics of the ED nurse role, such as the unpredictable nature, that she was otherwise intimidated by. Beth stated:

I applied to work in the ED because I actually liked it ... all the different things you get to see, it’s very exciting, it wasn't as chaotic, you don't see people actually running around like they do on TV. I found it manageable, and the acuity was less than what I expected ... [but] it is still not as routine as the units, you know with all the morning care, [administering] medication ... it's better ... in the emerg anything can happen. Through her experiences, Beth realized that the ED offered her a variety of challenges while also being predictable enough that she would know what is expected from her role during patient care. Having to engage in patient interaction and providing hands-on personal care to patients was not a concern any longer. Presumably, providing personal hygiene care to patients was not a focus of her experiences in the ED and therefore would not pose as much of a problem for Beth. 
In the next section I present the narrative ideas that emerged from subtext of data that pertained specifically to Beth’s experiences in caring for patients with dementia.

\section{Narrative Ideas-Dementia Specific}

The proceeding narrative ideas are derived from subtext of interview data that related to the research question: How do emergency nurses narrate their experiences of caring for patients with a diagnosis of dementia?

\section{Dementia: Experiential Knowledge}

During her years of nursing experience, Beth gained an informal understanding and knowledge of dementia. Through reading Beth’s interview transcript it was evident that her understanding of dementia was focused on symptoms and the healthcare provider's perception and care of the person with dementia, rather than focused on knowledge of evidence-based behavioural interventions. Beth describes an experience of a patient with dementia who came into the ED after experiencing a fall. Beth stated:

... She was confused, just by the way she was talking to me, she didn’t seem oriented, and she kept calling out a name and trying to leave, trying to climb out ... I kept telling her to stay, so she wouldn't fall ... I put the bed lower and the table across the stretcher too.

This excerpt illustrates the limited dementia knowledge Beth has. Beth does not allude to the possibility of a psychological reason or meaning behind the patient's attempts to leave or climb out of the stretcher. She attempts to control the patient's behavior in order to maintain the patient's safety, but Beth does not articulate a deeper understanding of the meaning behind the behaviour or how to address it in a meaningful and non-restrictive manner. Extending a table 
across the stretcher rails for purposes other than to have a meal, restricts the patient's movement and is therefore considered a form of restraint (Huabin, Lin, \& Castle, 2011)

This next excerpt validates that most knowledge of dementia stems from the repeated experiences of caring for persons with dementia, rather than from formal education. When Beth was asked to describe her knowledge of dementia, she described it as: "confused elderly people who are unable to follow instructions. They're difficult, like getting a history from them, like during an assessment finding out what happened and what's wrong." It is possible that Beth has based her knowledge on her experiences of what it is like to care for them rather than what her knowledge is about dementia in general. The meaning of dementia is inferred with references to her unpleasant experiences. Because of this lack of knowledge and understanding of how to provide care that is personalized for the patient with dementia, Beth centres in on the medical condition more confidently, as she has the knowledge and skill to address a fall and not a dementia. She stated: "I just treated her like a regular patient ... I knew she was confused, I didn’t treat her like someone with dementia, and [instead] I treated her as a fall.” It is also plausible to interpret this as Beth being cautious not to stigmatize the patient with dementia; rather she prefers to treat all patients equally. However, this perpetuates a non-person-centred approach to dementia care.

Beth took the following photographs to further describe her experiences of caring for patients with dementia. I have included two out of six images, namely images 6 and 7 are representative of her knowledge about dementia. 


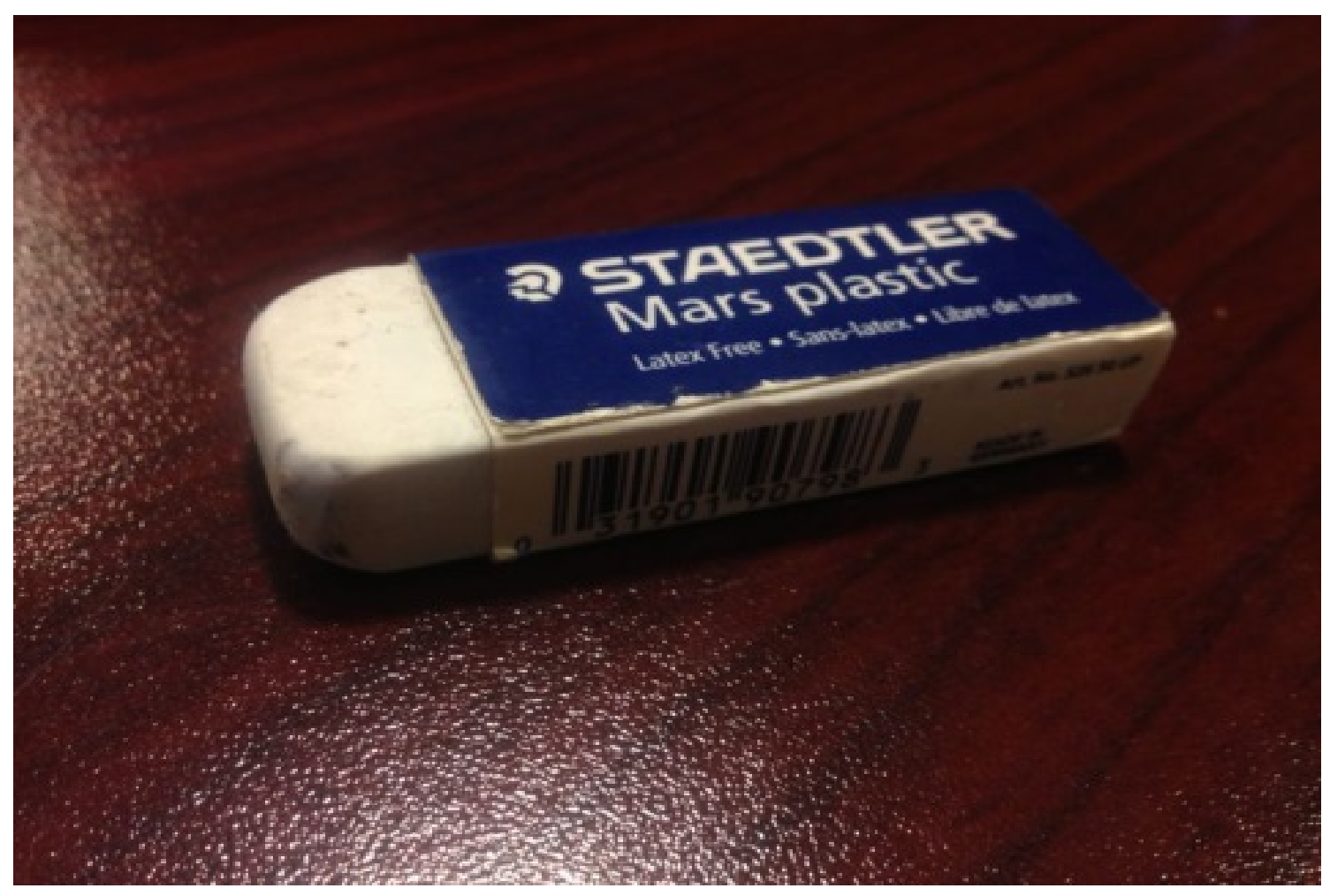

Image 6: Erased Memory

With dementia patients their memory or their mind is kinda erased . . . so you're working from a blank slate ... we have to dig for information cause they aren't able to provide us with as much, they are very limited ... a lot of them lose their memories so its like erasing their knoweldge ... they forget short term memory ... sometimes they yell out names from the past, asking for their parents or home, so they do remember stuff, its just long term.

The element of memory loss in dementia is central to Beth's experiences and knowledge of dementia syndromes.The difficulties and challenges in eliciting information from a person with dementia is a major part of her experience and knoweldge of dementia. The notion of digging for information is indicative of how challenging and exhausting it is for Beth to look after the dementia component of the emergency patient with dementia, hence why she addresses the 
medical needs first. Furthermore, the inability to understand this patient and address her/his needs does not fit with Beth’s appreciation for clear cut, black and white interventions and outcomes. In image 7, knowledge and understanding of dementia is derived from practitoner’s perceptions and judgements. Beth stated:

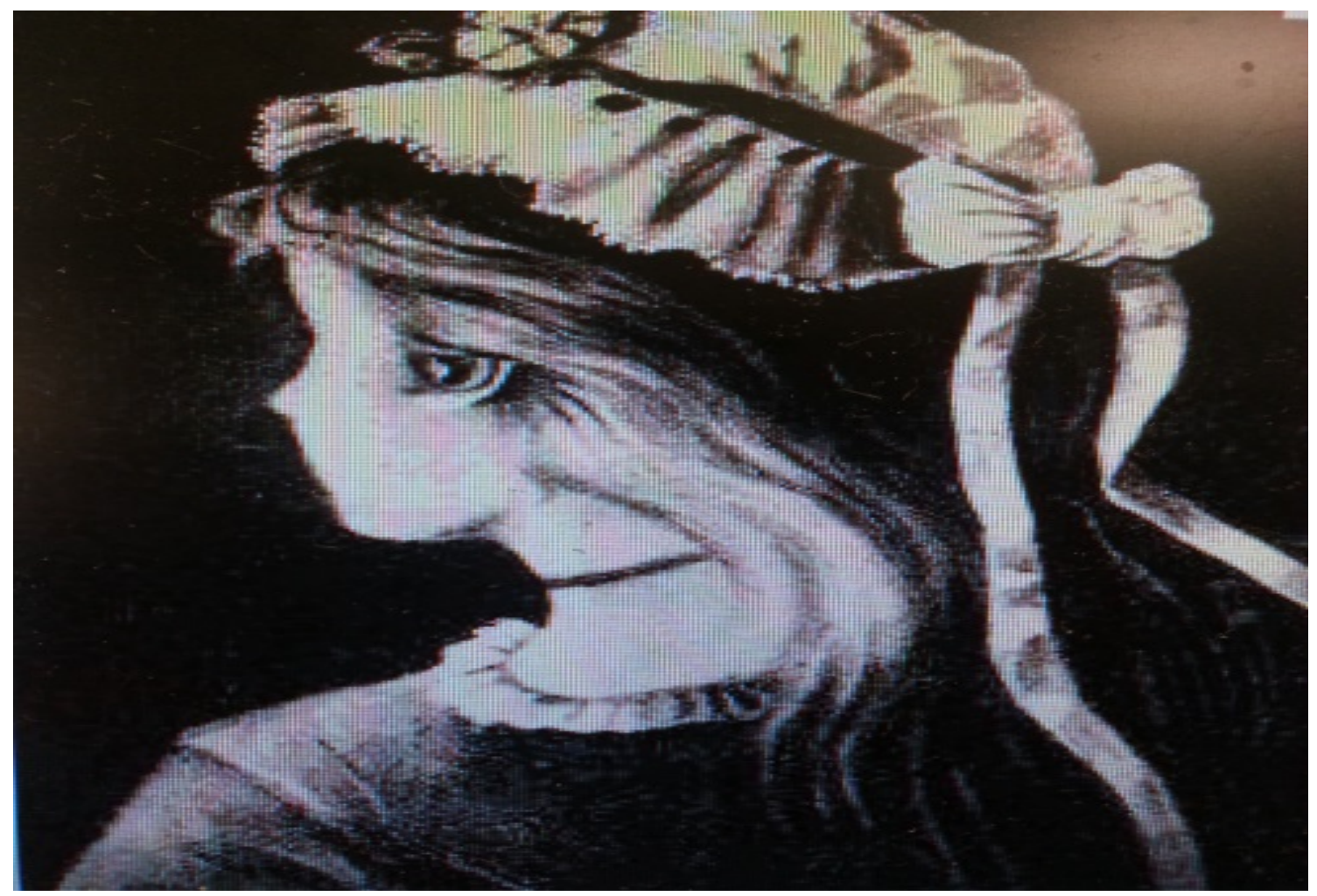

Image 7: Clinical Perception of Dementia

Everyone can look at one photo but perceive things differently. There are different meanings to it. You can look at this [photo] and see an older woman or a young girl. So with dementia patients, the first thing we think of is they are confused or agitated, just everything negative about them . . . it's our assumptions and perceptions we kinda label them a certain way.

Beth confirms through image 7 that the knowledge of dementia held by practitioners is derived from their day to day experiences and perception of how the patient with dementia in the ED is 
judged. There is an ED cultural understanding of dementia as Beth explains, referring to the common understanding, judgements and stigmatization of patients with dementia that are held by ED practitioners. Symbolically, this portrait of a young girl turning into an old woman represents how the mind may interpret the image in the most familiar and easier way first. Similarly, the mind can interpret the person with dementia from the most common pathological lens, viewing the person as a disease rather than a person first. It is easier to interpret and label the person based on her/his behaviour rather than learn about her/him and the characteristics of her/his illness. There are several optical illustration photogprahs available; however, it was interesting that Beth chose this one to describe her experiences. This image shows a variation from being a young woman to an older woman, the variation is in some way representative of the person with dementia whose cognition can fluctuate between what is acceptable cognition from a young woman to what might be expected from an older woman.

\section{Person-Centred Care: The Impossible}

Beth describes person-centred care as a sort of luxury in the ED, something that is time and patient dependent. If it is something that can be provided quickly and efficiently to a patient who can understand, communicate and receive the care then providing person-centred care is possible. Beth captured image 8, 9, 10 and 11 to express her experiences related to personcentred care and the disparities in the provision of healthcare to people with dementia in the ED. 


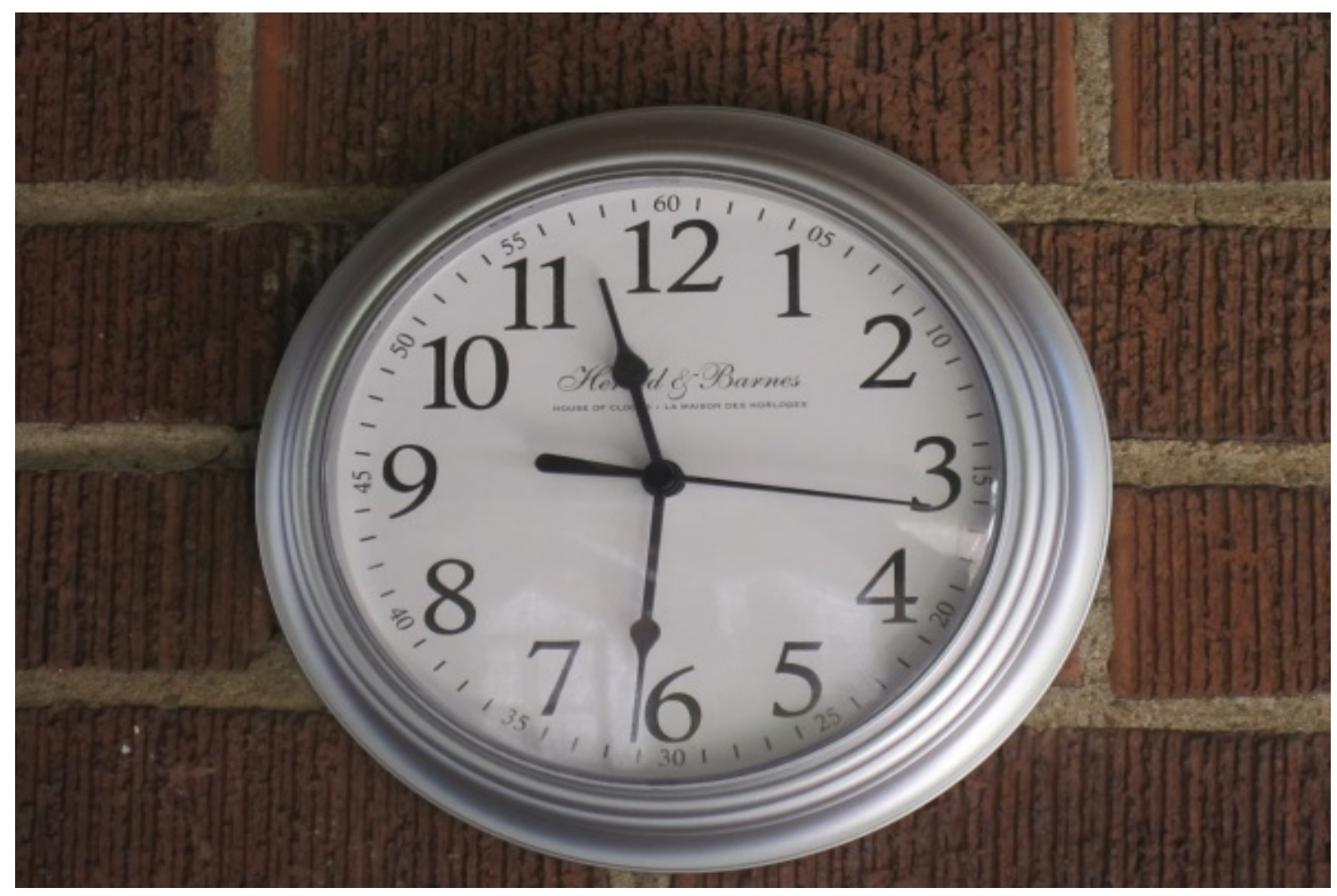

Image 8: Time

This tells me I have to be more patient with these patients ... they require a lot more time ... just spending that extra few minutes to keep them calm, or to communicate with them, take my time to use certain words that they understand, or speak in more simple words, and just try to listen to them. [The clock] also means time in general . . . the amount of time these patients spend in the emerg . . . they have to wait many many hours, we often don't feed them or change them or give them fluids . .

Beth describes her various experiences with the concept of time. Providing tailored care to the person with dementia requires astute communication skills, patience and time. Beth identifies some of the necessary approaches to communication when speaking with older patients without cognitive impairement. She stated: "with elderly patients, I use a louder voice, slower speech, more hand gestures ... your communication style is different ... ” For Beth, having reciprocal 
communication with an elderly patient without cognitive impairment is something she can achieve with minor communication style changes. However, if that patient has dementia, Beth is not as inclined to dedicate the time. For Beth, verbal communication becomes one way rather than mutual, as she believes the patient with dementia is unable to verbalize their needs or affirm the care they are receiving. A patient without cognitive impairment can easily verbalize and articulate their needs, whereas the patient with dementia may use less words and therefore may require greater dedication of time, effort and knolwedge from the practitioner. In an ED this is the type of person-centred care that Beth wants to apply to her practice, but faces a dilemma when time does not permit.

There is a disparity in care provision for the patient with dementia in the ED. Beth exemplifies this in image 9.

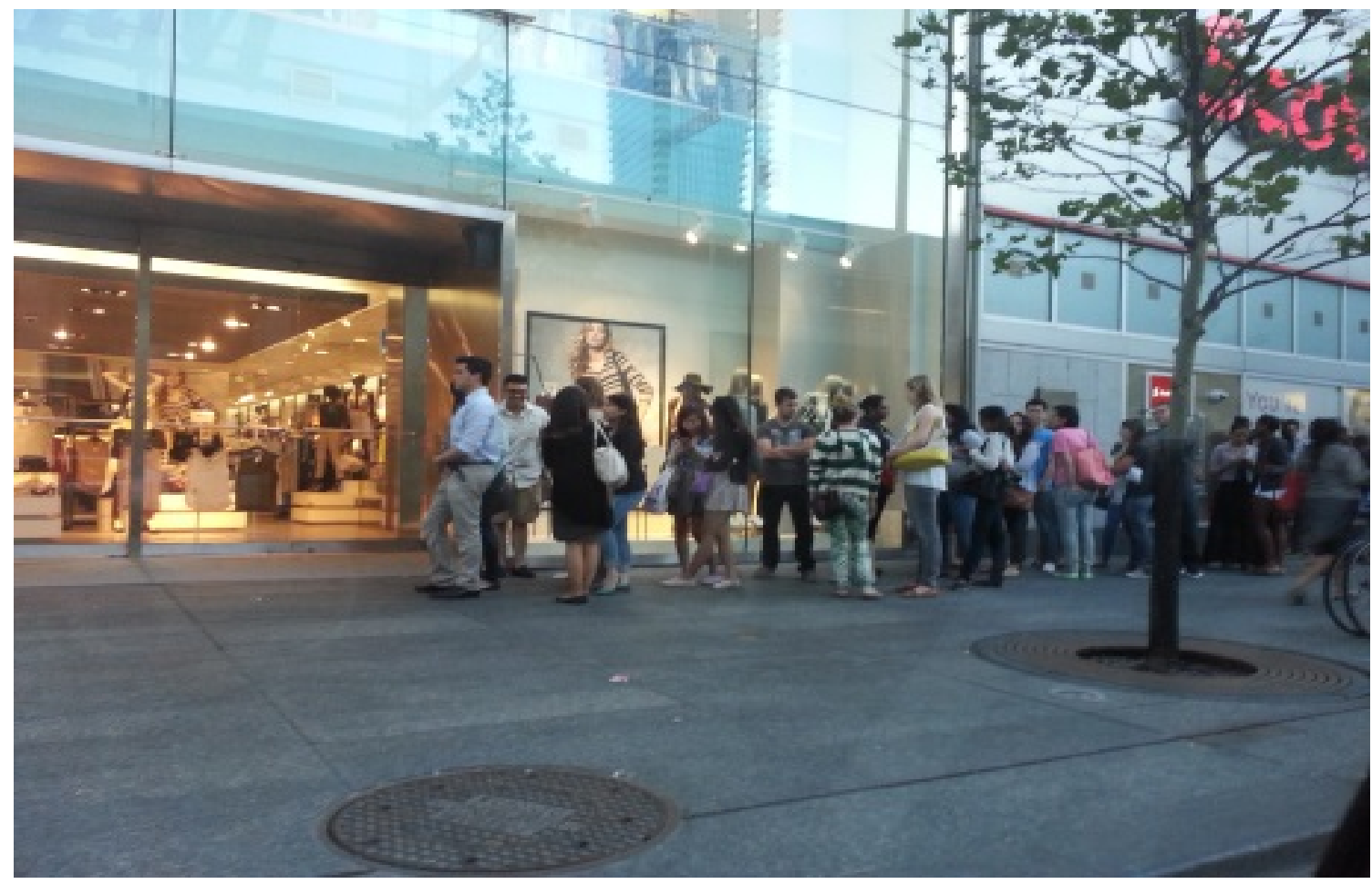

Image 9: Wait Times 
Beth described image 9:

They are often not the first patients to be picked up and seen right away ... they do have longer wait times than regular patients, the non-dementia patients, especially when there is no one else with them ... the emerg is a rapid assessment place and if you require that much more time to spend with them, then it takes you away from other stuff, so they have to always wait at the end of the line. Even nurses they would probably see the patient last, they wouldn’t be priority ... you would eye ball them ... they wouldn’t be the first to see even if they had chest pain, you don’t even know how reliable their story is, so you wouldn’t take them as serious as your other patient.

Image 9 and related excerpt represent the bias and discrimation that has become part of the culture in the ED where the dementia patients' reliability is questioned. There is a threat to the patient's physical health if they are not being seen in a timely manner. Person-centred care is heavily compromised because the time required to care for the patient with dementia does not align well with the fast pace nature of the ED. Waiting in the back of the line, being marginalized and unattended to has become part of the routine practices in the ED. In the case of the patient with dementia, they are viewed as unable to verbalize or complain about waiting, making it easier for practitioners to just "eye ball” them and return to them at a convenient time.

Image 10 further illustrates the threats to person-centred care in which physical restraints are used to save time and manage a heavy work load. 


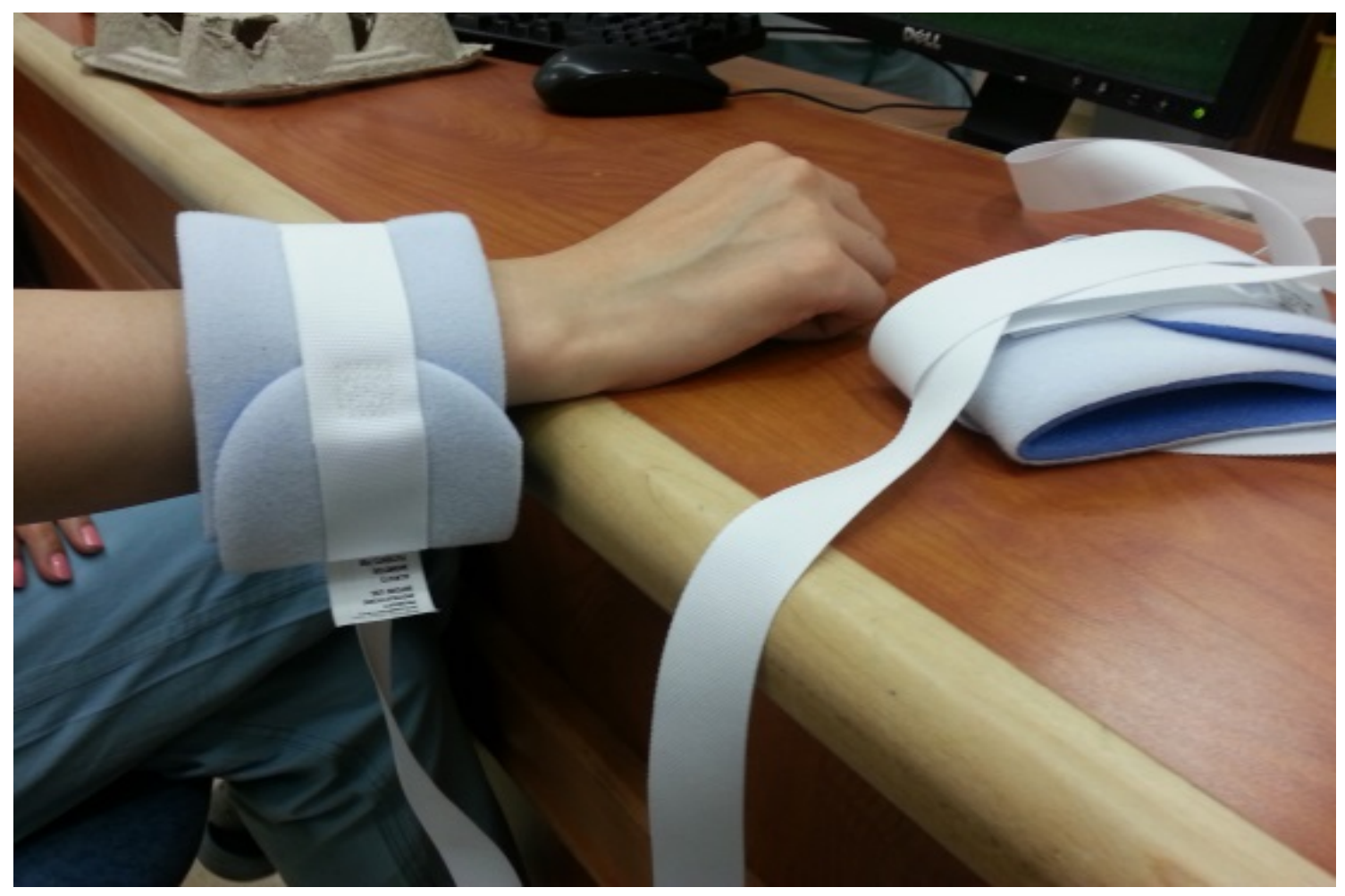

Image 10: Restraints: At Times Necessary

Her experiences reflect the mismanagement of patients with dementia through the common use of restraints as a form of minimizing patient activity, which interferes with the flow of work in the ED. This approach is staff centered rather than person-centered. Beth describes the experiences behind image 10:

I think with restraints all the times we choose to use it is because it's convenient for us, then we can have them stay in one place, while we do other tasks . . . we are supposed to only use them if everything else has [failed] . . . instead of understanding the underlying cause of their behaviour we just automatically say let's just put restraints to control their behaviour . . . but sometimes restraints can be helpful to protect the staff, if they are kicking or hitting you can at least have them held down for a bit and then give chemical sedation ... I think we are just finding the easy way out too. 
This excerpt depicts how person-centred care for the patient with dementia can be compromised in the interest of meeting competing demands. Beth faces a dichotomy, she understands and feels there are times when the patient is treated unjustly by being restrained; however, rationalizes these actions based on doing what is most efficient for workload as well as supporting staff safety. When I asked Beth to describe some measures she has taken prior to resorting to using restraints, she stated:

First I would tell the patient to do certain things, try many, many times, try to keep them on the stretcher, and they don't, then you would maybe like you know occupy them with other stuff like give them a drink or something to eat, then if it still doesn't work they're still agitated maybe put something over their bed, or tilt their bed so it's harder for them to get up. I don’t really like restraints that much.

Although Beth described the importance of assessing the reason behind behaviour, there is no indication that an attempt was made to address the patient's motives. Beth moves from identifying a problem to developing solutions without assessing the patient. The solutions are essentially similar and can be viewed as restricting the patient and lacking a person-centred approach.

\section{Emotional Labour of Work}

Beth shared an experience of caring for a patient with dementia in the ED, which involved significant emotional effort. For Beth, care of the patient became physically and emotionally taxing when she would not see positive outcomes from her continued efforts. As mentioned, Beth is a problem-solver and she seeks to attain answers. Beth shared:

I had one patient that came in from a nursing home she had a fall. They brought her in the room and she was seen by the doctor, she was very agitated at first ... she wouldn’t sit 
still ... she was confused, just by the way she was talking to me, she didn't seem oriented, and she kept calling out a name and trying to leave, trying to climb out ... I kept telling her to stay, so she wouldn’t fall ... I put the bed lower and the table across the stretcher too... you kind of have to stay with her and it takes away from everything else ... it's very difficult, she wasn't able to comprehend and follow instructions ... so we didn't think she was going to go to imaging, we did give her I believe Ativan, or something just to settle her... I went on break, then someone said she was on the floor. She ended up being on the floor, she had fallen from the bed.

Here, Beth does not achieve positive responses from the patient even after constantly attempting to negotiate with the behaviours of concern. Beth stated: "I was annoyed a little bit; I knew she had difficulties, it’s just difficult to keep going to her.” As a result of the negative patient outcome, Beth felt inadequate in her approach to managing the patient's behaviours. She stated: "I [wonder] was there something I could have done to prevent it. I feel like I should have done something to prevent her from falling." She further stated: "I feel like maybe it was my fault ... that is the last thing you want to happen.” This experience illustrates that Beth feels responsible for this patient's outcome. Beth further captures this experience in image 11, reflecting her focus on implementing tasks so that she can administer tests and procedures. 


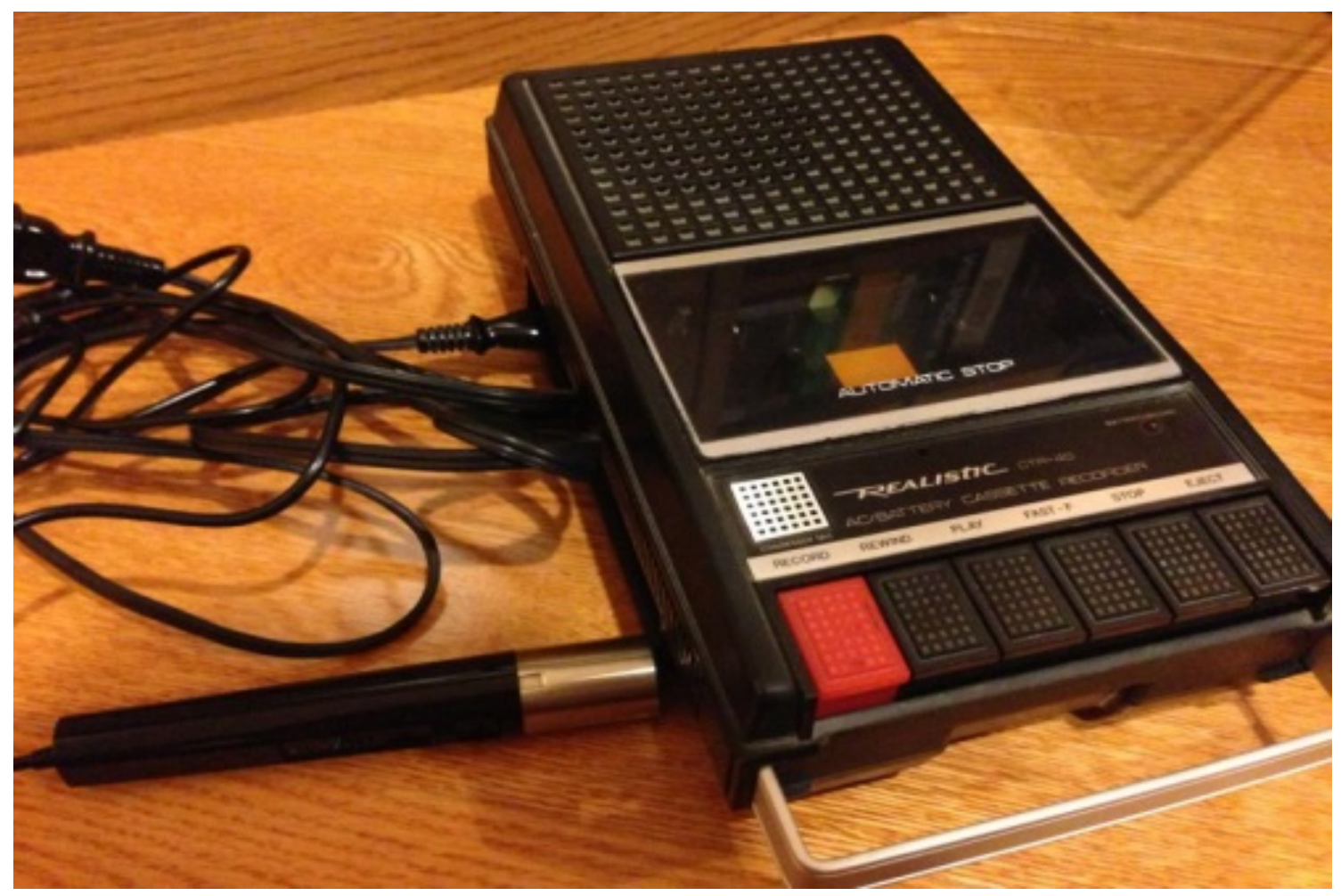

Image 11: Constant Repetition

Image 11 is symbolic of the consistent efforts Beth describes when communicating with the patient with dementia. Beth stated:

You often have to repeat yourself time after time, sometimes they still don't get it . . .

talking to them is difficult . . . like you're trying to ask them something, buy they're not on the same page as you. You can't have a conversation with them. Then if you try to do certain tests or blood work, they are not very compliant; they start to get agitated and don't want to be held down ... or sedated.

As reflected in Beth's description of the photograph, the care of and communication with the dementia patient is repetitive and appears as futile in her narrative. When caring for a patient with dementia, the result of spending added time with them and attempting to communicate with them may not immediately be rewarding. In the excerpt below, Beth interestingly assigns value 
to having a conversation with the patient. Beth experiences a dissonance between the care she strives to provide her patient with dementia and her limitations. She stated: "there is not enough time to do everything, there is not enough time to spend with certain patients, even though we would like to, things move too quickly, there's too much other stuff to do.” These experiences illustrate the emotional labour of work when caring for the patient with dementia.

\section{Support \& Appreciation}

Beth identifies measures of support and appreciation, which can mitigate the challenges she confronts when caring for the patient with dementia. Beth identifies various gaps in resources that she believes her leadership team should invest in. She stated:

There is a lot more to learn about patients with dementia and how each patient can present differently and it is sometimes hard to adequately care for these patients in the emergency room because we don’t have the right resources, time or information.

As reflected in this excerpt, Beth identifies the need for support to be able to manage competing demands in order to provide person-centred care to patients with dementia. Also, she refers to the need for more education about dementia to improve her assessment and care of the person with dementia.

Beth also defines family caregivers as an important support system to mitigate some of the challenges experienced. With reference to the incident of her patient falling out of a stretcher Beth stated:

It would have been nice to have someone like a family member or caregiver with her, maybe it would have settled her a little bit, just having someone with her that she recognizes, maybe she wouldn’t be as agitated seeing someone familiar . . . then I wouldn’t have to constantly go up to her and keep making sure she wasn’t climbing out . 
.. family could also provide history and the [patients] baseline, because you can't ask the patient, they don’t really remember everything.

As reflected in this excerpt, Beth identifies that families can provide an element of familiarity for the patient as well as a history about the patient and reduces the nurse's workload of keeping the patient safe in bed. Appreciation from patients is important for Beth who strives to provide the best care she can. She stated: “... patients thanking you, that appreciation and gratitude from them makes me want to stay, feels like I'm doing something ... like helping them.” This is a sort of reward, if the patient shows indication that they are pleased then it encourages Beth to withstand the challenges and continue to provide care. In the next section, I provide an analysis of categorical-form as it relates to Beth's narratives and experiences in caring for the person with dementia in the ED.

\section{Categorical Form Analysis}

I have used the Alzheimer Society Person-Centred Language (2012) and the Alzheimer Society Shifting Focus: a guide to understanding unpredictable dementia behaviour (2013), to analyze the cognitive elements of Beth’s narratives. In the next section, a categorical-form analysis was carried out on subtext of the interview data to provide a deeper understanding of the cognitive and emotional elements (Lieblich et al., 1998) of Beth’s story.

\section{Cognitive Analysis}

Language is a powerful tool and depending on how it is used it can elicit varying emotions. It can also affect the way we perceive and interpret behaviours of dementia (Alzheimer’s Society, 2012). Being sensitive to person-centred language in dementia can help maintain an individual's dignity and respect while allowing practitioners to move away from reducing the person down to a disease. An analysis of the language used by Beth to describe her 
experience with dementia reveals that her knowledge of dementia is limited and painted with language that is not person-centered. This excerpt is taken out of Beth's narrative when she described her experience of the patient with dementia.

She came from a nursing home ... she was agitated ... we gave her Ativan to settle her ... she was confused ... she didn't seem oriented ... she kept calling out a name ... kept trying to leave ... she was trying to climb out ... I put the bed lower ... I put the table across the stretcher.

Beth's choice of language in her narrative as well as her interpretation of the event is problematic. The term nursing home denotes illness and loneliness, is medically-oriented and historically associated with negative images of older people, a more person-centred language would be Long Term Care home (Alzheimer’s Society, 2012). The term agitated is one of the Behavioural and Psychological Symptoms of Dementia (Alzheimer’s Society, 2012). When I read this term, my immediate emotional reaction was that it disturbed me, and it seemed that it affected Beth in some way. Beth does not provide contextual details that would help identify the needs-based etiology of the behaviour, as is recommended. It is considered best practice to describe behaviours to give specific examples, otherwise it would suggest that the behaviour is a result of a problem with the patient and removes any alternative explanation for the changed behaviour (Alzheimer’s Society, 2012).

In the same excerpt, Beth described the patient as settled after being medicated. The term 'settled' makes me think the patient may have been disruptive or irritable, and the nurse heroically saved the patient from some discomfort by medicating her which resulted in the patient and nurse both being 'at peace'. Settling the patient could potentially be used to support the staff versus supporting the patient. Furthermore, being confused and not oriented are terms 
used by Beth to represent the memory loss associated with dementia. In describing the patient's mental status, it appears that the patient has some fault in the matter when in fact these are normal symptoms of dementia and can at times be meaningful of a particular need. Beth narrated that the patient kept calling a name, trying to leave, and climb out of the stretcher. When I first read this passage I interpreted the behaviour as disruptive and non-compliant, I sensed the frustration that Beth must have experienced. However, in the context of dementia, the patient's behaviours could be a reflection of an unmet need (Alzheimer's Society, 2013). The patient may have been calling for a familiar person, she might have been frightened in her unknown surroundings, she wanted to be home where there was familiarity, and maybe she was climbing out because the stretcher was strange and uncomfortable.

To address the patient's behaviour, Beth put a table across the stretcher and lowered the stretcher. A first impression might reveal that Beth was protecting the patient, and in this case these were likely her intentions. However, it also suggests that the behaviours were a problem of the patient instead of a symptom of dementia that warrants further assessment. In the next section I analyze the emotional content of Beth’s narrative.

\section{Emotional Analysis}

In this section I discuss three methods of analysis that provide insight into the emotional content of Beth’s narrative including linguistics, paralinguistics, and formal elements of speech.

\section{Linguistics: the said words.}

In order to illustrate the emotional content of Beth's experience I composed a poem to represent, verbatim, the language of her narrative account of what it was like to care for a patient with dementia. 


\section{Beth’s Experience with Dementia}

She had a fall at the nursing home

She was agitated on arrival

I administered Ativan

She Settled

She is confused, she is not oriented

She is calling out a name

She is trying to climb out

I said stay in the stretcher

I put the bed lower

I put a table across the bed

She Fell

This poetic reiteration of Beth’s experience describes her difficulties in managing a patient with dementia who was constantly attempting to get up from their stretcher. The poem is essentially divided into two stanzas to illustrate that Beth’s first attempt of medicating the patient only worked for a short time. The patient's behaviours persisted, and regardless of the interventions Beth employed, the patient eventually fell. Beth has read and validated the interpretation and presentation of this poem to be reflective of her narrated experience.

\section{Paralinguistics: the unsaid words.}

To analyze the paralinguistic properties of Beth’s dementia narrative, I listened to the audiotape and reviewed my field notes paying special attention to expressions, gestures, volume, intonation, vocal quality, and pauses in speech.

In the office where I interviewed Beth, she sat close to me; however, not facing me, with her hands folded over her lap and legs crossed, she faced the wall. I sensed that she might have been uncomfortable and shy. Beth's body language and gestures remained the same throughout the interview, minimal eye contact and a closed body language. I did not note any changes in her expressions or gestures while she narrated particularly emotional elements of her story. 
After listening to Beth’s audio-recorded interview several times with particular attention to her narration of her experience with the patient with dementia, I discovered interesting speech patterns. Beth’s recollection of the event was vague and required additional prompting to accumulate a more detailed story. There is a break in Beth's voice and a change from speaking at a normal pace while stating "she was seen by the doctor ..." to slowing down and becoming more quiet while she approached the following utterance: "she was very agitated at first ... " I sensed that Beth approached this thought with some caution and hesitancy. I wonder whether she was determining whether agitated was an appropriate term to use. A second and even more dramatic change in her voice occurred between the following two utterances: “we didn’t think she was going to go to imaging ..." which belonged to her normal tone and quality, and then there was another break in her voice, it suddenly paused when she said "we did give her, I believe Ativan ...” In listening to the audiotape recording, and reviewing my field notes, I heard and sensed another moment of hesitation, as if Beth was once again monitoring her words and being cautious of what she shared with me. I gather that Beth did not want me to judge her inappropriately based on the language she used. I understood her hesitancy because we were discussing sensitive topics.

\section{Formal elements.}

The linguistic features reveal emotional detail about Beth and her experience in caring for this patient with dementia. I reviewed a subtext of the transcript as it related to Beth’s experience of caring for this patient with dementia based on a modified list of elements provided by Lieblich et al. (1998). I concentrated on the chronology of events and repetition of words or utterances to explore the deeper meaning of Beth’s experience. 
As Beth shared her story, there were several times where I had to prompt her to share greater detail about her experience. In reviewing her transcript I found that Beth’s story had an interesting chronology of events. For example she stated:

I had one that came in from a nursing home and she had a fall, so they brought her and I don't remember . . . but after she was in the room and she was seen by the doctor ... she was very agitated at first, so we didn’t think she was going to go to imaging to cat scan . . . we did give her I believe Ativan, or something just to settle her a little bit, I had that done and then went on break, and someone said she was on the floor.

What I heard and saw in this narrative were large leaps in time, which Beth likely did unintentionally. In this passage, the patient arrived, was seen by a doctor, became agitated, was given sedative medication and then Beth went on break. A leap in time or breaking the chronology of events can indicate an attempt to avoid recalling a difficult time (Leiblich et al., 1998). After further prompts Beth provided some more detail about this event:

She was confused, just the way she was talking to me, she didn't seem oriented, and she kept calling out a name trying to leave. I kept telling her to stay; she was trying to climb out. . . I wanted her not to fall so I kept saying stay in the stretcher and you're going to fall I would put the bed down lower, I put the table across the stretcher too ... I knew she had difficulties.

The missing utterance from the first narrative is that Beth had a suspicion this patient might have sustained a recent fall; she even attempted to prevent the fall by restricting the patient's movement. From the first passage it seems that Beth only medicated the patient then left for a break. In fact, Beth initiated several nursing interventions and also assessed the need to institute a fall prevention technique. Albeit, it is unclear that Beth identified the implementation of these 
strategies in alignment with the organization's best practice policy. In the first passage there was no way to decipher that this patient was at risk for falling. After several prompts, Beth described a difficult experience.

Further analysis into Beth’s experience of this patient with dementia reveals another interesting pattern in the repetition of words and phrases. For example, Beth iterates the word 'fall' 5 times and the phrase 'she was on the floor' 3 times. According to Leiblich et al. (1998), repetitions reveal that the narrative has an emotional charge. In this case it would be highly indicative of Beth's negative experience around the patient falling. I previously mentioned that Beth felt responsible and perhaps even guilty about the patient falling, so much so that Beth even left that piece of the narrative out of her initial recollection of the event. It would make sense that this was an emotional moment for her and she felt highly responsible as her true recollection reveals she went on break with the awareness that this patient was at risk for falling.

To further illuminate and understand the findings from this study, in the next section, I provide a comparison of the similarities and differences between Beth and Eva's content analysis. 


\section{CHAPTER 6}

FINDINGS: EVA \& BETH: A COMPARATIVE ANALYSIS 


\section{Eva and Beth: A Comparative Analysis}

In keeping with the methodological approach I subjected the text to additional readings and analysis to evaluate the relationship between both participants' themes. After I reviewed Eva and Beth’s transcripts individually, I then conducted a simultaneous comparison of their narrative ideas and utterances. I found that some distinct and recurring similarities and differences between them emerged. As I further compared and analyzed interview transcripts it became more apparent that who Eva and Beth are as individuals has influenced their experiences with and perspectives of persons with dementia. These are important to highlight in order to achieve a deeper meaning of the shared stories.

\section{Inclination for Acute Care}

Previous experiences encouraged Eva and Beth to seek a career in nursing. As a lay person working in a hospital, Eva witnessed a dying patient and felt incompetent that she could not save this person's life, therefore went into nursing with a commitment to save lives and heal people. Whereas Beth discovered a passion for healthcare through her many volunteer experiences. Both Eva and Beth had an opportunity to be exposed to the ED as clinical students through their undergraduate nursing program. Their clinical experience confirmed for both of them that a career in the ED was what they wanted. Specifically they both enjoyed the variety of patients and all the new learning opportunity that the ED provided.

“[In the ED] it was the variety of patients ... the continuous hands-on learning” (Eva)

"I applied to work in the ED because I actually liked it ... all the different things you get to see, it’s very exciting” (Beth). It is interesting to note the similarity in their imagination of an ED career prior to being ED nurses. Both participants used comparable 
vocabulary to describe their image of the ED as being like a television show, highly acute, and unpredictable.

"I thought it would be more trauma cases, like the TV shows ... all the blood, shocking, [and] asystole ... just the excitement of it.” (Eva)

"I thought it was going to be like crazy ... from watching it on TV, like the Untold Stories of the ER.” (Beth)

The most evident difference between Eva and Beth was their desirability of the unknown and unpredictability in the ED. Eva enjoyed the unpredictable, acute and chaotic nature of the ED, whereas Beth was more drawn to the predictability of patient issues and outcomes and the more managed flow of a work environment. They stated:

“... all the blood, shocking, [and] asystole ... just the excitement of it ... but a lot of times it's more the clinic cases, or the elderly people with [chronic] CHF or COPD ... it’s challenging if someone comes in with something I don’t know about.” (Eva) "I was not looking forward to it, cause I thought it was going to be chaos, difficult and crazy ... you're the first person that people [see], I think what if I don't know what to do.” (Beth)

Through these passages it is evident that Eva, unlike Beth, is more inclined to handle and manage the patient with an acute and unpredictable course of illness. Beth reveals her attraction for consistency and predictability, and even managing illnesses that are more chronic in nature, as she would be familiar with the care she needs to provide.

\section{Dementia: Experiential Knowledge}

Eva and Beth developed their knowledge of dementia primarily through their experiences in the ED. The variation of knowledge was rather interesting and highlights the influence of 
individual character and personal preference on their developed knowledge and understanding of dementia. Both participants had a general idea of dementia, often associating it with memory loss, confusion and disorientation. Participants stated:

"She was confused, just by the way she was talking to me, and she didn't seem oriented . .. they are confused elderly people who are unable to follow instructions.” (Beth) “I guess around 7 o'clock [pm], that’s when you start to notice sundowning ... confusion would set it ... the patient had to continually be re-oriented ...." (Eva) One of the most prominent differences between Eva and Beth's knowledge of dementia is predicated upon their personal experiences. Also, their expectation from a nursing career seems to have influenced their experiences and thus their knowledge of dementia.

In general, Beth sees dementia through a more critical lens, focusing on the practitioner's preconceived judgments of the patient. This can in part be explained by Beth's indifference with managing either chronic or acute illness in the ED. Beth was not drawn to the acuity of the ED therefore managing the older patient with dementia was not a problem for her. Alternatively, Eva went into nursing to manage and treat acute illness and to save lives. The expectation Eva had from her career in nursing was not realized when she had to care for patients with chronic illness and older people with dementia. Thus, Eva's experiences and knowledge of dementia may have been described more negatively. The following statements support this tension:

"I was constantly repeating myself ... I hate to say it but it was like an attention-seeking behaviour ... if she genuinely was forgetting then I felt sad for her.”(Eva) "the first thing we think of is they are confused or agitated, just everything negative about them ... it’s our assumptions and perceptions we kinda label them a certain way.” (Beth) 
In the following comparisons it will become more evident how their past and personal preferences have shaped their experiences with dementia.

\section{Person-Centred Care: The Impossible}

There were striking similarities associated with Eva's and Beth’s understanding of person-centred care. For instance, both participants highlighted the significance of time limitations in the ED and how this subsequently affected their practice.

"Keeping the patient occupied has helped me with my workload ... gives you more time, you don’t have to be there as often ... in the ED you don't have the time ... instead you move them in the hallway where they are safe.” (Eva)

“... the emerge is a rapid assessment place and if you require that much more time to spend with them, then it takes you away from other stuff.” (Beth)

The idea of time limitations in the ED did not surprise me since I too had experienced this. I also found it interesting that both participants had developed their own approaches in managing the behavioural symptoms of dementia and felt strongly that their approaches were appropriate. They stated:

"My approach would be to take a Geri-chair, put it in front of the nursing station , and he would have been way calmer , [even] slept in the chair ... move them in the hallway where they are safe, give them sheets to tie ... that will keep them preoccupied. (Eva) "I would tell the patient to do certain things, try many, many times, try to keep them on the stretcher, and they don't, then you would maybe like you know occupy them with other stuff like give them a drink or something to eat, then if it still doesn't work they're still agitated maybe put something over their bed, or tilt their bed so it's harder for them to get up.”(Beth) 
Both of these passages reveal that knowledge of how to address behaviours of dementia is learned through experience, unit culture, and role modelling, since they make no reference to theoretical or evidenced-based standards for many of their practice decisions around mobility and safety.

Similar to my experience with Ms. Levine, I practiced nursing according to the expected culture, such as medicating the patient, as well as what I learned through my experiences as an ED nurse, rather than through evidenced based practice. Participants often identified these techniques as person-centred and justified their approach as being in the patient's best interest, keeping them safe, occupied, and calm. Eva and Beth had similar feelings with regards to the use of restraints. It is interesting to note a contradiction between their stated feelings about restraints in comparison to their descriptions of using restraints as a behavioural control in their every day practice, for example, using a Geri-chair or putting a table across a bed to limit the patients movement. They stated:

"Tying them up is inhumane ... they should be treated with dignity and respect ... I think sadly it's like putting them on leashes ... we just tie them up to know they are there, we can come back later when it is good for us.” (Eva)

However, Eva describes her own use of restraints, she stated: "My approach would be to take a Geri-chair, put it in front of the nursing station, and he would have been way calmer.” (Eva) "I think with restraints all the times we choose to use it is because it’s convenient for us, and then we can have them stay in one place, while we do other tasks ... I think we are just finding the easy way out too.” (Beth) 
Beth provided further discussion by examining nursing practice through a critical lens. She revealed some of the stereotypes and biases held by practitioners about people with dementia in the ED. She stated:

"They are often not the first patients to be seen right away ... they do have longer wait times than regular patients ... they require a lot more time ... they have to wait many many hours, we often don't feed them or change them or give them fluids.” (Beth)

Beth's narrative provided a more critical explanation of ED nursing practice. When she narrates her experiences she not only discusses her personal challenges of caring for patients with dementia, but, she also provides a lens with which to view the issues from the patient's perspective. Beth does this by scrutinizing and elucidating her own practice and that of her colleagues. She brought our attention to the disparities expeirenced by these patients in the ED.

\section{Emotional Labour of Work}

For both Beth and Eva the emotional labour of work developed as a result of constantly doing the same thing and not seeing positive results. Participants described varying emotions of sadness, annoyance and frustration, but knew they had to repress their feelings in the interest of the patient and continue to provide care. They stated:

“It’s very difficult, she wasn’t able to comprehend and follow instructions ... you often have to repeat yourself time after time, sometimes they still don’t get it ... talking to them is difficult ... I was annoyed a little bit, I knew she had difficulties; it’s just difficult to keep going to her ... I feel like I should have done something to prevent her from falling ... I feel like maybe it was my fault." (Beth)

"At any given time [their mood can fluctuate], they can be pleasant then suddenly confused, mean, and agitated, and you're like what the heck is going on, it challenges you 
as a nurse ... if they are completely at a state of gone, nothing there, no orientation, it's frustrating, you know you keep trying, and trying and trying but I don't get anywhere ... It doesn't matter what I do or say nothing is going to change it ... I feel sort of like a slave, sometimes angry, I can’t say what I'm thinking.” (Eva)

These narratives illustrate that as ED nurses and individuals who measure their accomplishments through tangible outcomes, Eva and Beth are dissatisfied and feel somewhat inadequate in their care of patients with dementia. In reflecting on my own experience with Ms. Levine, I too felt frustrated with my inability to understand my patient and meet her needs. Regardless of their individual characteristics and expectations from their career both Eva and Beth derived similar solutions to address some of the frustrations they have experienced when caring for patients with dementia.

\section{Support and Appreciation}

As participants uncovered some of the challenges they experienced in the provision of care for patients with dementia they also discovered what could help alleviate or resolve some of those challenges. I was not surprised to see that their focus slightly shifted in one direction or another, based on their particularly different experiences. For Eva, a predominant issue for her was that her patient did not have family support which resulted in the patient requiring physical and emotional support from Eva. Just as her narrative account was infused with issues of family involvement so were her solutions.

"the family would just rely on me to turn her and get her back into bed ... the patient was more combative while the daughter was there $\ldots$ at one point the [daughter and neighbor] stepped out of the room and said to me it's your job, you go and take care of her ... when family members are there you want to rely on them to help you.” (Eva) 
For Beth, her narratives were saturated with issues of time and communication with respect to caring for the person with dementia. It seems that Beth discovered many of the challenges she was confronted with were related to the inability to communicate with her patients, and recognized that it takes more skill and time to have effective dialogue with the patient with dementia. As expected, Beth’s solutions to mitigate some of her challenges were related to having more time and information supports.

"It is sometimes hard to adequately care for these patients in the emergency room because we don’t have the right resources, time or information.”(Beth)

Finally, both paricipants valued being recognized for their hard work. Receiving gratitude was a way to kept them motivated and feel their efforts were worthwile. As individuals who like to see the fruits of their labour, It was not surprising that they appreciated reassurance they were doing a good job.

\section{Comparative Analysis Summary}

Overall, participants narrated common issues around their experiences in caring for patients with dementia. These included time constraints, inability to provide person-centred care, inadequate support from family caregivers, constantly attending to patients in the midst of competing priorities, and the inability to acquire tangible behavioural outcomes despite their continued efforts. To address these issues they have identified the need for greater family carer support, more time from other activities to attend to the needs of patients with dementia, more information on how to care for patients with dementia, and more appreciation and recognition to mitigate the challenges they experience in caring for patients with dementia. In the next chapter I will further interpret the findings of this study and discuss them in relation to current literature. 


\section{CHAPTER 7}

DISCUSSION 


\section{Interpretive Discussion}

I begin this chapter by providing an interpretive discussion of my research findings and how it fits with existing literature. I answer my research question, "How do emergency nurses narrate their experiences of caring for patients with an underlying diagnosis of dementia”? In addition, I discuss the findings in terms of the secondary questions that guided this study, "How do dominant discourse and power relations between nurses, their work environments and the healthcare system shape nurses' stories and their practice of caring for this population?” I then identify the study's strengths and limitations, recommendations for future research, education, professional practice \& policy, a conclusion, and epilogue.

I began this thesis with my own story as it related to my experiences in caring for a patient with dementia. I explicated how the challenges and tensions I experienced as an ED nurse informed the direction of my thesis. I wanted to not only share my experiences with others, but also explore the experiences of other nurses. I wanted to reveal any issues and begin to understand the dilemmas nurses, and their patients, confront every day. As I reflected back on my own story as well as those of Beth and Eva I have come to understand that we shared many common experiences. In order to appreciate the meaning of these findings, I have divided this interpretive discussion into two sections: 1) the glamourized ED and 2) the culture of the ED.

\section{The Glamourized Emergency Department}

The glamourized ED is often depicted as a high acuity setting where patient care activities are episodic and technical, and treatments of patients revolve around life-saving procedures and rapid clinical decisions and actions. I chose to call this the glamourized ED because these characteristics are continuously presented in the media as admirable and interesting and have become the common image and ideas held by viewers. These are the 
dominant discourses of emergency nursing. Many times the realities are omitted in these portrayals, leaving even new nurses to select this appealing and glamourized area of nursing to establish their careers. I will explore the idea of the glamourized ED by looking at: images of an ED; characteristics of the ED nurse; and the exemplary dementia care nurse.

\section{Images of an ED}

When we were new graduate nurses, Eva, Beth and I had a predetermined image of the ED, where it is chaotic, traumatic, technical, fast-paced and highly episodic and acute in nature. Patterson, Bayley, Burnell, and Rhoads (2009), conducted a mixed methods study to understand how new graduate nurses perceive the orientation program and emergency nursing at the beginning of their career and six months later. In this study, new graduate nurses saw the ED as fast paced and busy. They described emergency nursing as constantly changing, unpredictable, challenging, intimidating, scary, intriguing, and exciting. Similar to my research, the authors note that the complex and dynamic nature of emergency nursing may be positive factors that influence graduate nurses to decide to work in an ED setting (Patterson et al., 2009).

For Eva, Beth and I as clinicians, these notions were derived from stories we heard from other nurses or the portrayal of the ED through popular media channels, including television drama shows. While Eva and I were drawn to the high level of acuity, Beth was first intimidated by this aspect. Eva and I were drawn to the ED to save lives, to be in the centre of action and to feel heroic by using our highly skilled and technical interventions. In the study by Nystrom et al. (2003), nurses described the most stimulating events in terms of acute medical interventions, especially when it concerned saving patients' lives, while caring for older people with psychosocial problems was perceived as boring. It is not to say that these acute elements do not exist, but, it is not the predominant activity in the ED. 
In my study, I found that perception of the ED nurse's role did not always match reality. Similarly, Nelson and Hillan (1995) found that 77\% of student nurses surveyed cited the media as their main source of information about Accident \& Emergency nursing. Furthermore, these students viewed acutely ill patients in an unstable environment as satisfactory (Nelson \& Hillan, 1995). For Eva, Beth and I, the realities of the ED began to set in once we established our careers in the ED. The ED was not always chaotic and episodic, it was dynamic in a sense that acuity levels varied. However, we would often find ourselves managing chronic conditions. The importance of good communication was paramount as we attended to many different types of patients, including those with dementia. Similar to our experience, Nelson and Hillan completed another questionnaire to determine whether student nurses’ perceptions had changed after gaining experience in an Accident \& Emergency department. It was found that $90 \%$ of participants reported a higher number of patients with minor trauma and a relatively low number of emergency cases. However, these nurses maintained a positive attitude about emergency nursing despite knowing the predominant ED activities were not highly acute rather minor. Similarly for Beth, who adapted to the ED role, she realized that the ED was not as overwhelming and chaotic as she once feared. Instead, there were more stable and non-traumatic patients and as the nurses in Nelson and Hillan's study maintained a positive attitude, so did Beth, who eventually enjoyed the predictability. On the other hand, Eva preferred to work in an ED as she imagined it to be acute, episodic, and chaotic.

\section{Characteristics of the ED Nurse}

As reflected in the narratives of Beth, Eva, and I, we were drawn to the fast-paced and technical nature of the ED, treating illness and observing tangible and well-defined outcomes of our care. These narrative findings are supported by a study examining the culture of the ED 
(Person, Spiva, \& Hart, 2012). Staff members were observed and interviewed and results demonstrated that staff felt a sense of reward when they were able to intervene for a patient and visualize a positive patient outcome (Person, Spiva, \& Hart, 2012). This sense of reward that comes with intervention that results in positive outcomes was also demonstrated in this study with Eva and Beth. Furthermore, the Emergency Nurses Association (1999) defines core characteristics of the practice of ED nursing as: triage and prioritization of patients, emergency operations preparedness, the stabilization and resuscitation of patients, crisis intervention for unique patient populations, and the provision of care in uncontrolled or unpredictable environments. These characteristics are reflected in my own personal story. I was attracted to and idolized Susan, my ED mentor, for her ability to multi-task and prioritize, as well as her knowledge, confidence and efficient nursing skills. These were some of the qualities I admired and aspired to have as an ED nurse. Similarly, Eva and Beth were driven by such motivators as having clear-cut solutions, being skillful, and managing their workload. These characteristics and expectations are in contrast to what is required and necessary when looking after the patient with dementia in the ED.

\section{The Exemplary Dementia Care Nurse}

While it is important to have skills and attributes that are well aligned with managing acutely ill ED patients, it is equally important to have a unique set of skills and attributes while caring for the ED patient who has an underlying diagnosis of dementia. In a busy ED environment where nurses must process orders in a time efficient manner to attend to the physical needs of patients, there is often little room to possess and demonstrate effective dementia care (Fessey, 2007; Borbasi et al., 2006). As in the narratives of Beth, Eva and I, our practices were not focused on the dementia related concerns of our patients. Instead we were 
dedicated to managing the fall, the infection, and the acute confusion that our patients presented with. According to Cunningham and Archibald (2006), focusing nursing interventions on the medical needs of the patient is a result of having limited understanding of the needs of people with dementia. Therefore, acute care nurses need to acquire additional skills and expertise to care for individuals who present with dementia in addition to their acute physical condition. This closely exemplifies Beth's reflection of her experience when she noted ignoring the patient's dementia and shifting her focus onto something she was familiar with, such as the patient's fall.

As demonstrated in Eva and Beth's narratives and photographs, their knowledge about dementia influenced how they perceived and cared for their patients. Kang et al. (2010) found that personal attitudes held by practitioners about patients with dementia influence job satisfaction and perceived competence providing dementia care. That is, they found that nurses working on medical wards had significantly more positive attitudes than those working in surgical wards. However, the authors note that results may have been influenced in part by the social system structure in Korea, where older age is highly honored and respected. The authors attributed the difference in attitude between nursing units to greater geriatric education on medical wards, less technical equipment used in medical wards, and having less responsibilities associated with high-tech interventions in medical wards. These findings are reflected in the current study. Eva's feelings and attitudes seemed to fluctuate when describing her experiences with patients with dementia. She described a negative experience when caring for a patient with dementia who entered the ED, while the patient with dementia in the community was illustrated more positively. Nurses with better understanding and training demonstrated favourable feelings towards people with dementia and perceived increased competence providing care to person's with dementia. 
Contrary to my findings, Nolan’s (2006) study, which sought to illuminate nurses’ experiences of caring for older persons with dementia in an acute hospital setting, found that perceptions of the patient with dementia as a person did not diminish with admission to the acute context. The nurse skillful in dementia will understand that repetitive requests are a form of communication of some need the patient is attempting to express (Alzheimer's Society, 2012), rather than attention-seeking behaviour, which associates the behaviour as burdensome and is blaming.

Eva, Beth, and I were inspired to work in an ED where we could provide care to patients with life-threatening conditions in a fast-paced environment. However, an exemplary dementia care nurse in an acute care setting will also need to possess characteristics and skills that are not necessarily aligned with the critical and fast-paced nature of the ED. Researchers have noted that skillful assessment and communication is paramount when caring for the person with dementia (Borbasi et al., 2006; Cunningham, 2006; Hoe \& Thompson, 2010; Moyle et al., 2008; Nolan, 2006). In the study by Nolan, nurses described the importance of bonding and relationship building with the patient with dementia to establish trust and rapport. They also found that for bonding to be successful it would require persistence and time by the nurse, and were easier to achieve with patients who were "pleasantly" confused.

As reflected in Eva's, Beth's and my narrative, we were often unable to establish a bond with our patients who presented to us with behaviours of dementia. This inability may be attributed to our lack of knowledge on how to effectively communicate and build trust with the patient with dementia, and the inevitability of having to manage multiple competing demands in a limited time frame (Nolan, 2006). The nurse who exemplifies person-centred care to patients with dementia will have a comprehensive understanding of how to communicate with the person 
with dementia (McCloskey, 2004). Normal communication patterns need to be modified to meet the needs of the person with dementia (McCloskey, 2004). For instance, nurses need to allow more time for the person to respond, speak slowly and rephrase statements that are not understood (McCloskey, 2004; Thompson, 2010). This approach takes time, and as ED nurses, Beth and Eva did not initially recognize that mastering these skills and dedicating so much time for non-physical needs would be part of their role.

As I reflect on my own experience, I idolized Susan, not because she had astute communication skills with patients with dementia, but, because she was rapid in her patient assessments, had confidence, and was highly technical. Although these skills are important, they can sometimes be at odds with the care patients with dementia require. In order to provide good care to patients with dementia, it is important to be able to recognize the triggers, meaning and emotions behind the behaviours (Alzheimer’s Society, 2013; Cunningham, 2006). Interpreting and addressing the needs of the patient can take time and patience, requiring nurses to modify their usual pace of activities. According to McCloskey (2004) the way in which nurses approach the patient will be mirrored by the patient with dementia who is sensitive to non-verbal communication. For instance, if the nurse is calm and has a gentle demeanor then the patient with dementia may emulate these behaviors (McCloskey, 2004).

In the ED, practitioners are constantly in a hurry to complete tasks. In my narrative I spoke about the difficulties I experienced in managing my workload while caring for Ms. Levine. The continuous movement and attempts to take Ms. Levine's blood and do an electrocardiogram in a timely manner likely evoked negative feelings in her. I spoke quickly to her and constantly waved my arms around while completing my technical tasks. I treated her as an exemplary ED nurse might: efficiently, timely, technically and with her physical needs as my priority, rather 
than an exemplary dementia care nurse. Because of our experiences, the glamourized image that Eva and I held about a career in the ED became apparent as we each noted it was not as technical and acute in nature as we once imagined. Rather, we had to learn how to adapt to a variety of patients who presented to us and at any level of acuity, even those with underlying cognitive impairment. These patients required the same exemplary quality of care and expertise as patients that do not present with dementia,

\section{The Culture of the ED}

In this section, I draw out findings closely informed by Critical Social Theory, which is the theoretical lens that guided this study. I provide an interpretive discussion of the findings to uncover how dominant discourse and power relations between the environment and the healthcare system and Eva, Beth and I, has shaped our stories and our practice of caring for a population of ED patients with an underlying diagnosis of dementia.

The number of people living with dementia worldwide is currently estimated at 35.6 million (WHO, 2012). In light of this overwhelming statistic, it is surprising that EDs generally have not yet adapted healthcare practice to be more inclusive in addressing the needs of this subpopulation of patients. The current culture and priorities of the ED are generally not aligned with providing person-centred care to a population of older patients with dementia. Through our narratives, Beth, Eva, and I, have identified several barriers to providing dementia care that is person-centred, including limited education and knowledge, a lack of time and support systems, and how these have been influenced by the biomedical model of care and a dominant discourse of efficiency evident in our nursing practice. In the following section I discuss these barriers. 


\section{Knowledge Deficit}

A considerable amount of what Eva, Beth, and I learned about caring for the person with dementia was through our experiences with patients rather than formal education. Even orientation and education is shaped through a dominant discourse that is reflective of a medically-based ED where acute illness is treated and chronic disease neglected. Beth narrated on several occasions that patients with dementia were routinely stigmatized as being confused and agitated individuals and these are the perceptions of healthcare professionals. Similarly, in a study on the care of older people with dementia in an acute care hospital setting in the United Kingdom, Cowdell (2010) found that most of the nurses' encounters caring for patients with dementia were based on their own philosophies rather than on evidence. Also, Travers, Beattie, Martin-Khan and Fielding (2013) conducted a survey study about specialist dementia RNs’ attitudes towards dementia care and training. It was commonly reported that a high level of dementia knowledge was important and positive associations between providing dementia care, self-confidence in caring for people with dementia, positive attitudes towards people with dementia and recent participation in dementia education were made (Travers et al., 2013). In the study by Cowdell nurses reported more confidence in providing physical care than psychosocial care for patients with dementia.

As I cared for Ms. Levine, I was perplexed as to why she was resisting the care I wanted to provide her with. I did not understand nor expect her behaviours to escalate to the point of aggression and climbing out of the stretcher. I was confident that I was trying to help Ms. Levine. I was carrying out my medical duties to identify any physical reason for her ED visit. Upon reflection, my nursing practice was strongly shaped by a medical discourse of assess, diagnose and treat. My experience is similar to what the nurses in the study by Nystrom et al. 
(2003) reported, when they discussed caring encounters with their patients with dementia, it was often in reference to practical things such as giving information or helping them to the bathroom. According to Cunningham (2006), nurses must be skillful in recognising early signs of distress in patients with dementia, as it could be the precursor to the development of responsive behaviours. Once responsive behaviours ensue, it is important to understand the underlying cause (Cunningham, 2006).

Eva's, Beth's, and my encounters with our patients with dementia were often narrated as disorderly and negative, and predominantly related to not having the knowledge about how to either prevent or address the responsive behaviours of dementia. For instance, the responsive behaviour of repetition was interpreted as "attention seeking” by Eva. According to the CohenMansfield Agitation Inventory, the behaviour the patient presented with is better recognized as repetition (Cohen-Mansfield, 1997), which can be a reflection of feelings of insecurity or loss, loneliness, separation from a loved one, under- or over-stimulation, and/or the inability to express a need (Alzheimer's Society, 2013, p. 7 ). Eva's narrative reflected that she may have lacked the knowledge to assess the broader context of the patient's behaviour; therefore, she misunderstood the patient's constant demands for toileting and water as an attention-seeking behaviour of dementia. Consequently, Eva would bring the patient water and toilet her frequently which she reported made the experience challenging and frustrating. With additional dementiaspecific education and departmental resources, Eva would be able to implement alternative meaningful activities and therapy, such as word games, books, puzzles even music therapy. Similarly, Dupuis et al. (2012) found that all resident behaviours reported by long term care staff were filtered through the lens of pathology. Their behaviours were attributed to the dementia 
disease process and broader social or environmental factors were overlooked, thus having implications for the quality of care patients received.

My study reflects the way that dominant discourses in the ER shape one's practice and decision-making process. Beth and I shared similar experiences with a patient who presented with agitation and was constantly attempting to climb out of their stretcher. There was uncertainty about the reason for the patient's behaviour. Beth and I were overwhelmed, and as a result of not having better solutions we sedated our patients with medication to ensure they were not agitated and instead cooperative enough to be put through necessary diagnostic testing and assessments. Eriksson and Saveman (2002) mirrored these findings in their study on nurses' experiences of caring for patients in an acute care setting. The nurses in this study described having to medicate the patients with responsive behaviours to make it possible to manage difficult situations and have time for other patients (Eriksson \& Saveman, 2002). In contrast, Nilsson et al. (2012) found that, although hospital staff reported not having guidelines or protocols for the care of older patients with cognitive impairment, they still provided the best possible care and treatment to their patients. The authors note that these findings demonstrate the limited awareness staff members have of the unique needs associated with people who have cognitive impairment, which risks compromising the provision of high quality person-centred acute care. Similarly, Beth and Eva adapted various solutions, which they learned through their experiences, to reduce or prevent responsive behaviours, including the use of a geri-chair, or situating a table over a stretcher, changing communication style, and even offering the patient sheets to tie. With more education and infrastructure supports Beth and Eva may have had the potential to become even more creative in the development and delivery of person-centred behavioural interventions. 
A limited knowledge and understanding among nurses of how to respond to responsive behaviours of dementia can result in sub-optimal care and restraint use (Fessey, 2007; Martin, 2002). In a study estimating the use of different types of physical restraints and their associations with falls and fractures in a sample of Long Term Care residents it was found that the use of chair restraints, such as a geri-chair, was associated with higher risks of falls among residents with Alzheimer's Disease (Huabin, Lin, \& Castle, 2011). Conversely, in a two year retrospective study that examined the effects of chemical and physical restraints on elderly patients in an ED, Swickhamer, Colvig, and Chan (2013) found that elderly patients who received restraints in the ED for both behavioural and medical purposes, had no documented adverse outcomes such as problems of elimination, aspiration, skin breakdown, injuries or falls. These findings were based on a general elderly patient population and not necessarily those with $\mathrm{AD}$, who may be at an increased risk for confusion and subsequent falls. In addition this study did not explicate the duration of time a patient was in a physical restraint, which could ultimately be a predictor of an adverse event. Findings from this study seem to support the use of restraints in the ED by nursing staff. However, given the limitations of the study, it is difficult to decipher if its use would in fact produce adverse events to patients with dementia.

Like many nurses, Eva and Beth used restraints in the form of a geri-chair and by placing a table across the patient's stretcher with the intent of maintaining the patient's safety. Eva described having to place the patient in a geri-chair to be able to attend to other activities without worrying that the patient may climb out of a stretcher or fall. While Beth put a table across the patient's stretcher to restrict the patient's movement out of the stretcher also preventing a fall. This practice was also found by Lejman, Westerbotn, Poder, and Wadensten (2013), wherein RNs saw belts, tray tables and bed rails as a necessity for senior safety reporting that the 
consequences of falls and injury were much worse than the consequences of physical restraint. These activities speak to the limited time available in an ED culture where activities related to curing the patients immediate medical need takes precedence over managing dementia-related behaviours. Again the dominant discourse and culture of the ED influenced nursing practice. Decision making around how patients with dementia should be managed was favorable for the department and practitioner and not the patient with dementia.

Participants developed interventions based on their experiences as opposed to empirical literature. Providing a patient with sheets to tie as a form of distraction is something Eva learned to do through her experiences as a nurse. She was unable to articulate any evidence-based rationale for offering a patient with responsive behaviours sheets to tie. In fact, her distraction is not consistent with the existing evidence. The literature shows that activities should be meaningful to the patient to allow the patient to be engaged and function to their full physical and mental capacity (Hoe \& Thompson, 2010; Warchol, 2006). Prevention and early identification of responsive behaviours is important to minimize the use of chemical or physical restraints. A better understanding of the reason why these patients were attempting to climb out of their stretchers, based on a thorough and knowledgeable assessment, can lead to individualized interventions that keep the patient safe without having to use restraints (Martin, 2002).

Some general communication strategies in preventing responsive behaviours, according to the dementia literature, is to stay calm, speak slowly and clearly, minimize extraneous stimulation, avoid sudden movements, sit at eye level with the patient, be consistent, provide reassurance, and modify the environment to what calms the patient (Alzheimer’s Society, 2013; Hoe \& Thompson, 2010; Weirather, 2010). Although these are important, Hoe and Thompson 
(2010) explain the importance of tailoring communication strategies to meet the patient's needs and cognitive ability and understanding. Beth had some knowledge about how to communicate with the patient, she stated spending an extra few minutes with the patient to keep them calm, communicated with them using certain words that they understood, and listened to the patient. Although these are some of the strategies outlined, Beth was unable to provide evidence-based rationale for her decision to use the stated techniques over other ones. When she exhausted her limited knowledge resources of how to minimize the patient's responsive behaviour through communication, Beth medicated the patient. She even mentioned ignoring the fact that the patient had dementia and focused her care efforts on the patient's medical needs related to the fall. In the study by Cowdell (2010), many nurses felt that they were ill-prepared to provide care to patients with dementia. These nurses compensated for lack of expertise by concentrating on what the author quotes as the 'bed and body' framework, which focuses on the physical care needs of patients rather than holistic, person-focused care (Cowdell, 2010).

Providing dementia specific education and training to nurses is generally not a priority of the ED. It is evident that as a result of the knowledge and information deficit, nurses in this study experienced challenges in managing responsive behaviors and subsequently patient's quality of care was compromised. Beth's patient suffered from a fall and was medicated as was Ms. Levine, while Eva's patient sat in a geri-chair or engaged in meaningless activities. As nurses, we provided the best care we could within our scope of knowledge about dementia, which evidently was inadequate. Nurses are required to advocate for their patients and adhere to a set of practice standards, but, many of the activities and priorities in the ED are incongruent with the standard of nursing care required by patients who have dementia. The ED functions within a larger biomedical, efficiency-driven, and acute care discourse. As part of this system, nurses 
learn to function and provide the best possible care within these models of care, which ultimately affects them and their patients, in many ways. As I reflect on my own experience, I was informed by a senior mentor that I had to embrace the culture of the ED: I had to be fast and efficient, think and respond to situations quickly and always be ready for any unpredictable event. However, I never learned how to manage responsive behaviors of a patient with dementia.

\section{Time Deficit}

Working within time constraints of the ED was recognized by Beth, Eva and I as a prominent barrier to providing person-centred care to patients with dementia. Eriksson and Saveman (2002) found nurses felt pressured by the organization to reduce care time; they felt there was not enough time to communicate with the patients with dementia. Moreover, Nystrom et al. (2003) equated the activities carried out in the ED to a conveyor belt; they had to process tasks quickly and efficiently, and the concept of care had meaning only in practical terms. Eva, Beth, and I, identified time as both a necessity to the provision of quality care and as the most common barrier, and when time was limited it would also affect our performance and selfevaluation as an ethical and good healthcare provider. Similar to our own feelings, Nolan (2006) reported study findings that nurses expressed feelings of guilt, challenge and difficulty when they described the limited time available for caring activities of the patient with dementia.

When time permitted, on a medical ward, prior to her ED career, Eva was able to provide tailored care to a patient with dementia. The patient was provided with a pen and paper to engage in letter writing, an activity that was meaningful to the patient. Eva understood the patient's needs and extended care beyond her technical nursing duties to meet those needs. The patient in the ED, who presented with agitation, was given sheets to tie, as Eva noted keeping the patient engaged in any activity helped alleviate some of the time pressures and allowed her to manage a 
heavy workload. Similarly, Eriksson and Saveman (2002) found that nurses' work was performed according to specific routines, and the patient with dementia was said to demand too much time and interfered with the routine work and nurses' workload. What could alleviate some of these demands is the employment of patient observers or constant observer. A patient observer, commonly known as a 'sitter' has a dedicated role to assist nursing staff by providing close one-to-one monitoring of adult patients who may be confused (Carr, 2013). Although having someone with the patient can be beneficial for both the patient and nurse (Sandhaus et al., 2010), this is not always readily available or feasible for the ED (Carr, 2013). It may be more cost-effective for the ED to apply physical restraints to restrict a patient's movement than to hire an individual to sit with the patient to monitor their safety. These practices are aligned with the dominant discourse and of the ED. As the participants and I narrated, nurses have the autonomy and freedom to make evidence-informed decisions about whether or not to utilize restraints on patients who may interfere with typical ED workflow or deemed unsafe to themselves or others, as was Ms. Levine and Beth's patient. Given the limited resources available in the ED to address responsive behaviours, it is not unusual that nurses rely on restraints when in difficult to manage situations.

Patients with dementia are typically restrained against their will and restraints are used as a measure to save nurses time from continually having to monitor their safety. Eva recognized this as problematic practice, articulating that it strips the person of their dignity and respect, nonetheless, it is routine and unchallenged practice in the ED. Borbasi et al. (2006) found that nurses often justified using restraints to protect the patient's safety as they were unable to continuously attend to them while under other pressures. In my own narrative, when I had to restrain Ms. Levine, I felt similar to Eva; I did not agree with the practice of restraints, I even felt 
guilty and sad for my patient. However, I attributed the inhumane actions to the necessity of having to meet the patient's physical needs while ensuring that workflow was uninterrupted; otherwise, this would have slowed down other processes and perhaps even prevented me from attending to my other patients that night. These findings are similar to that of Eriksson and Saveman (2002), where nurses described ethically difficult situations as ones where they had to administer high doses of sedative medications against the patients' will in order to manage their workload and attend to other patients. Despite knowing that practice can be improved, it is possible that participants believe that non-person-centred practice in the ED can be justified and acceptable in the presence of time resource limitations. This predominant social discourse is manifested in practice, despite knowledge, ethical viewpoints, and evidence to the contrary. These are the power relations that nurses are confronted with in their work environment, and in a broader context that includes the healthcare system.

One of the predominant issues participants raised in caring for the patient with dementia was the amount of time required to manage these patients' behavioural, psychological and communication needs. For example, Cowdell (2010) found that nurses focused on the patient's physical needs as this met certain standards of practice in their workplace. Additionally, the physical need is consistent with the dominant discourse that shapes the culture of an ED and time is allotted for these types of activities. The laborious work required in constantly attending to the repetitive questions and requests of the patient with dementia interfered with Eva's and Beth's competing work priorities and were the predictor of a stressful shift. Tensions occurred for all of us as we practiced according to a set of organizational and departmental values and beliefs that embraced productivity and efficiency and technicality while risking a compromise in personcentred care delivery, and these were not congruent with our own as professional nurses. 
Interestingly, Cowdell's (2010) research found that nurses caring for people with dementia in an acute setting were seen by others as relatively unskilled and not prestigious, and therefore they did the work that needed to be done, which was a routine approach to the delivery of care. This speaks to the higher value placed on providing technical care in a time efficient manner while providing person-centred care to a patient with dementia is ranked lower as a skill in an ED culture. In Nolan's (2006) study, nurses reported the importance of bonding and trust building with the patient with dementia as a prerequisite to the delivery of ethically justifiable care, and given time limitations in an acute care setting, this was otherwise difficult to attain.

In the ED, every minute is valuable, as Eva, Beth and I have narrated in our experiences. The patient with dementia was often a challenging experience for us. We were not able to address their needs sufficiently, and because their responsive behaviours were escalating and interfering with other processes we were compelled to somehow restrain the patients, whether this was through medication, physical restraints, placing a table across a stretcher or sitting them in a geri-chair. We all noted feelings of guilt, frustration, and challenge, especially when wanting to do more for the patients within a limited time frame and pressure to maintain a throughput of patients. According to Cunningham and Archibald (2006), there are political and economic pressures to move patients quickly through the system to ensure waiting times in the department are achieved. Anything that slows this process down could be open to scrutiny and repercussions. Therefore, minimizing interference from the patient with dementia was necessary to ensure other patients' orders were being processed in a time sensitive manner. The investment of time required to communicate with and gather a history from the patient with dementia does not match the priorities of the ED and therefore hinders the patient's care disproportionately. Beth often described the patient with dementia as being last in line; they had to wait longer to be seen 
by physicians and nurses when compared to patients without cognitive impairment. Moreover, this can also be explained in part because of a lack of knowledge practitioners have about how to manage the responsive behaviours of the person with dementia. Cunningham and Archibald (2006) confirm that challenges often arise when staff members have a poor understanding of the needs of people with dementia and the demands associated with meeting those needs in acute care environment. Similarly, Burgess and Page (2003) found staff may distance themselves from the person with dementia who requires their care because communicating with patients is discouraged in favour of completing non-patient related tasks as processes of a ward takes precedence. These patients slow down the typical rapid assessment and treatment that is common in the ED. According to Borbasi et al. (2006), managing patients with dementia was repeatedly described as complex and challenging, requiring more thought than for other patients, which deterred staff from caring for them, often leaving their needs overlooked.

Beth also described the patient with dementia as being last in line. This was common and acceptable practice in the ED, especially in the interest of time and productivity. These practices became part of the ED culture and routine practices. In Cowdell's (2010) study, nurses were reported to work in a "switched off" mode, meaning they provided care in a routine fashion because that was the way it was always done, they never questioned their practice. As Beth described, the patient with dementia could otherwise be left for considerable periods of time without attention to nutritional, toileting or pain needs. My experience reflects that of Beth's, Ms. Levine laid on a stretcher in the hallway for some time yelling for someone to take her back home, my mind intentionally muted her yelling in order to finish my tasks, because I knew if I had attended to her psychological needs, I would potentially not complete my other tasks, tasks that I knew were more important within an ED culture. As noted in our narratives, when we look 
after patients with dementia, their physical care takes precedence over their emotional, psychological and even social needs. In a study on the experiences of older patients waiting for ED treatment, it was reported that nursing care was mainly characterized as meeting medical and technical demands, that a medical-technical culture was dominant in the ED, and nursing care was observed to be significant only as preparatory work to prepare the patient for the doctor (Kihlgren, Nilsson, Skovdahl, Palmblad, \& Wimo, 2004).

As I cared for Ms. Levine that night, I followed my normal routines, every task and technical skill I performed was in preparation for the physician as they wanted the patients with blood results and an electrocardiogram ready to be assessed in a timely manner. It is evident that time, resource, and knowledge deficits have played a role in how nurses manage the patient with dementia. As noted by Cunningham and Archibald (2006), people with dementia do not respond well to a culture that conforms to rapid treatment protocols and to a dominant culture of curing the patient. It is clear that cultural and professional models of illness and care influence the delivery of healthcare. In a biomedical model, such as the one that dominates the current healthcare system, the patient with dementia is viewed in terms of their illness, and all symptoms and signs that arise are a result of illness (Wade \& Halligan, 2004). In this model, when the underlying illness is treated then it is expected that symptoms will also resolve. The patient becomes a passive recipient to treatment and if they want to get better, they must conform to treatment (Wade \& Halligan, 2004).

It appears that the participants and I experienced various tensions, challenges and frustrations as we provided care within these boundaries. Providing person-centred and holistic care on the one hand are incorporated into the mission and value statements of an organization, however, these are not imperative in the ED when it concerns the patient with dementia. Beth, 
Eva, and I recognized that the person with dementia in the ED experienced a loss of dignity and respect. According to Kitwood (1997), it is important that personhood is maintained in dementia, as it would allow for a psychological interpretation of the experiences of the person with dementia as well, thereby ensuring the individual is appropriately assessed and treated within a framework of humanism. In addition to the identified need for a more person-centred care approach to care, a strong support system from family caregivers and the organization is also needed.

\section{Support Deficit}

Eva and Beth often narrated the challenges inherent in eliciting information from the person with dementia, which resulted in the patient not being assessed and treated as efficiently and effectively as other patients might have been in the ED setting. The nurses in Borbasi's (2006) study reported that the inability of patients with dementia to provide a history added to the complexity of their care, and at times they were overlooked because of this. Similarly, the nurses in Nolan’s (2006) study reported the difficulties in gathering information from the person with dementia about their life history, likes, dislikes and routines, which would have otherwise helped them strategize individualized care. Similar to Eva and Beth, these nurses emphasized the importance of having family available who could provide insight into the patient's history and working with family members to establish better care for the person with dementia (Nolan, 2006). Having family caregivers available to provide a history and partner with professional caregivers to help in the assessment and management of the person with dementia is evidently an important support system for practitioners.

The patients Eva, Beth and I looked after were in distress, they were confused, agitated, and aggressive, likely because they were surrounded by unfamiliar faces in a place that was 
foreign to them. According to Borbasi et al. (2006), nurses reported that support of people with dementia requires dedicated family caregivers in a familiar environment; nurses described family as an asset and beneficial to the patient and staff. Beth and Eva suggested that if family caregivers were available they could have helped calm the patient when agitated, or provided security to the patient during treatment and medical care. Borbasi at al. (2006) reported that healthcare providers in the acute care setting described the environment as being strange for the person with dementia, very different, and even dangerous, and staff were not familiar with the patients, their normal routines, and how they coped on a daily basis. If family caregivers were available in the case of Ms. Levine, she may have not needed to be medicated; family members could have provided comfort and familiarity to Ms. Levine who may have been afraid as her environment and surroundings changed from a place of trust and familiarity to the chaotic and noisy ED environment. According to Moyle et al. (2008), the hospital environment produces multiple competing stimuli which can be disturbing to the person with dementia, triggering unwanted behaviours.

In order to manage her workload, Eva reported having to use ancillary staff to observe patients with dementia, as family were not always available and the patients required close supervision. In this study, it was found that the absence of family caregivers, coupled with the high workload of the nurse, sometimes put patients at risk of being restrained, medicated, or both. Participants narrated family support as being pivotal in the care of the ED patient who has dementia. Overall, it may have helped alleviate time pressures, improve the flow of their workload, assist in providing a baseline heath status of the patient, and ultimately improve the experience of the patient by offering the patient comfort and familiarity. 


\section{Strengths \& Limitations}

Like any type of research, narrative research, as a methodology, contains its own set of unique limitations and criticisms. As a result, I have employed various strategies to addresses each of these. Critical comments on narrative research often pertain to the authenticity and trustworthiness of data collected and the stories that are narrated (Holloway \& Freshwater, 2007). These elements of credibility are questioned as a result of the subjective and interpretive nature of narrative research. In other words, the data collected in narrative research are informed by both the participants' experiences shared, as well as the evaluation and interpretation of the data by the researcher. Because of these potential limitations, as the researcher, I have attempted to see, hear and interpret more than that which was directly captured in words (Holloway \& Freshwater, 2007).

Similarly to the problem of truth telling is that of the participant's memory of experiences. When participants were asked to recall a memory from the past they may have been selective in what they remembered as being most important at the time. In this study, the concurrent analysis of categorical-content and form provided both a systematic and multi-layered method of analysis of interview data, allowing me to analyze the deep meaning of participants’ stories and report data in a rich and descriptive manner, thus adding to the rigor and strength of study findings. Also, by subjecting data to a cognitive and emotional analysis, and by reflecting

on my observational field notes I was able to reveal some of the deeper meaning of linguistics as well as the unspoken experiences of participants. Furthermore, the use of photography in this study added an additional layer of explanation to participants’ stories and experiences and allowed me to be aware of alternative explanations, thus adding to the overall strength of interpretation of findings. 
There are also limitations inherent in the activities of the narrative researcher. For example, during data collection it is said that only specific elements from the participant's stories that have relevance or are related to the agenda of the researcher may be included in the analysis and unrelated elements may be left out (Holloway \& Freshwater, 2007). In this study, this was addressed in three ways. First, I compared and supplemented interview data with photographs from participants allowing me to make a more complete interpretation of the participants’ experience and eliminating the potential for important pieces of information being omitted. Second, I took final research findings back to study participants to ensure the accurate collection, interpretation and depiction of their experiences. During the second interview I had an opportunity to ask participants further questions to clarify or elaborate some of their responses and my interpretations of them. At the end of data analysis, emergent themes were also taken back to participants to be reviewed for accurate reflection of the meaning of their experiences. Ultimately these activities strengthened the width or comprehensiveness of evidence adding to the strength of this study (Lieblich et al., 1998). Third, throughout the research process I shared my ideas and interpretations with my thesis committee for their input and questioning. In this way I was prepared to justify my interpretive analysis and continuously demonstrate how research findings were grounded in the data, therefore, adding to the study's strengths.

Being both the researcher and an ED nurse could be interpreted as a potential limitation. However, in the qualitative paradigm a deep understanding of the phenomenon is the research goal. Therefore, situating the study in my clinical practice knowledge is seen as a strength of this study. In narrative research, the researcher is in continuous dialogue with the stories of participants. In keeping with the research paradigm I stated my positionality as a researcher disclosing where I stand and how my subjective experience provided an interpretive lens for this 
study. By being reflexive, and through maintaining field-notes, I have made explicit my own experiences, feelings, and emotions of the phenomenon, and how these may have influenced the direction of the research strategies and procedures. Moreover, being both the researcher and an ED nurse I was in a position to better understand and relate to the participant's stories, including some of their language and meanings. I had the ability to see the phenomenon from both a researcher and ED nurse lens adding to a more thorough interpretive analysis of findings.

Using CST as a theoretical lens to guide the processes of this research was an important tool. It allowed me to continuously critically analyze and interpret participants’ stories and experiences in relation to greater sociopolitical discourse. CST provided me with an ability to think deeply about participants’ responses. In examining their stories this way, I was able to reveal how factors such as time, lack of resources, lack of knowledge, and priorities of the ED culture interfere with the delivery of person-centred care to patients with dementia. This is also in line with the aim of narrative research, as it focuses on the subjective experiences of narrators, taking into account the wider societal and cultural experience. In this way, narrative and CST both seek to explore the deeper meaning of experiences situated within the tellers culture and social world (Leiblich et al., 1998).

\section{Recommendations: Research, Education, Practice and Policy}

\section{Research}

This study has added to a small and important body of research about the ED nurses’ experience in caring for patients with underlying dementia. Drawing from nurses' stories, many issues have been identified in this study making each of them amenable to further investigation, and thereby adding to existing literature. Future research is essential to explore whether knowledge and education enhancement in the area of dementia care improves nurses' experience 
in caring for patients with dementia. A study comparing the experiences of nurses with and without formal education could highlight the value and importance of education as a predictor of good or poor experiences in caring for the patient with dementia. This study can be extended to look at how nurses' knowledge and education impacts the patient experience. Since time was a significant predictor in the experience of both the nurse and patient further research is warranted to look into the effectiveness of having nursing aids or unregulated healthcare professionals in the ED and how this might impact care. Last, given the conflicting research presented on the adverse effects of physical restraints, a study examining the effects of restraints in the ED with special attention to the duration restraints are applied is merited. These studies can help answer some of the questions that continue to remain, while also confirming the importance of some of the strategies I will now highlight.

\section{Education}

In light of the growing number of persons with dementia, now more than ever, nurses need the appropriate knowledge, skills, and tools to be able to effectively and ethically care for patients with dementia who enter the ED. Without the education and training nurses will continue to provide care based on their experiential knowledge only, which can impede patient outcomes as we move further away from practicing according to principles of person-centred care. Education needs to extend through the continuum of nurses' experience, from nursing school and throughout their career. Starting early in the academic institution, more attention needs to be dedicated to including theoretical knowledge on dementia in undergraduate curriculum. In this study the two nurses denied any prior knowledge from their respective nursing schools. Furthermore, efforts should continue as students enter the workforce. In the workforce, education and training can then be more focused on the assessment and management 
of the behavioural and psychological symptoms of dementia. Nurses in this study struggled to articulate and manage these symptoms, leading them to unethical situations. Orientating nurses to the reality of a job in the ED is also warranted; that is, new nurses entering the ED should be aware of the array of dementia patients and behavioural situations that they may be confronted with, while also being given the appropriate tools to manage these situations. For example, initiating such programs as PIECES, PLST, and HELP for nurses working in EDs could provide important and necessary knowledge and resources. Even when nurses move from being novice to experienced, they face similar unpleasant experiences when caring for patients with dementia; therefore, education should be ongoing to provide updated best practice knowledge to staff (Burgess \& Page, 2003).

One tool that has been introduced and is currently being used in the ED I work in is Montessori Methods for Dementia (Elliot, 2013). Montessori principles are based on the original work and philosophies of Dr. Maria Montessori, a physician and childhood educator. The foundation of Montessori Methods is based on the philosophy that individuals should be as independent as possible, to have a meaningful place in their community, to have high selfesteem, and have an opportunity to make meaningful contributions to their community (Elliot, 2013), this is also in line with the person-centred care philosophy. In instituting Montessori Methods staff must learn about the person behind the dementia, that is, an assessment of their needs, interests, abilities, and skills should be determined. Then, with the right training, anyone can introduce and engage the person with dementia using appropriate and tailored activities that match the level of the individual's ability.

These activities are meant to purposefully engage the person to their full potential, stimulating their sensory, cognitive, social, cultural, and spiritual abilities. According to the 
Alzheimer's Society Person-Centred Care Framework (2011) activities can be an important way of helping a person with dementia to retain their independence. Activities are easy to institute and it is suggested that they decrease and prevent responsive behaviours of dementia (Alzheimer's Society, 2011). Training staff on how to use Montessori Methods is easy to do and requires only one full day of training (Elliot, 2013). Some Montessori activities that have been used successfully in our ED to decrease boredom and prevent responsive behaviours is sorting of pictures and items, playing cards, photograph books on hobbies and animals, large print word puzzles, painting and drawing, doll therapy, and word and picture matching. In adopting a more formal and evidenced-based tool to engage persons with dementia we can begin to move away from implementing meaningless and non-person-centred care activities such as tying sheets and folding towels, which does not support an individual to express themselves to their full capacity.

\section{Practice and Policy}

Nurses need support from their leadership team as well as the organization as a whole to improve their experiences and that of patients. In light of the major time constraints identified in this study, there are a number of ways to address this. First, it is clear that nurses need support staff such as an unregulated healthcare worker (e.g., a nurse's assistant or personal support worker, even a technician to do the electrocardiograms and lab work). Support persons could help the nurse with tasks that are not necessarily required to be completed by an RN. If nurses are not overwhelmed with various competing tasks, this can help them attend to those patients who require some more of their time. Second, the nurses in this study did not mention having any person or resource expert in geriatrics or who may have been familiar with dementia. The nurses relied on only their experiential knowledge. Therefore, introducing the idea of a geriatric nurse specialist would be a beneficial service to nurses while improving the experience of older 
patient's (Ryan, Liu, Awad, \& Wong, 2011). This person could be a valuable asset in addressing care needs of the elderly patient with dementia and a resource to nursing staff who may require education.

Developing recommendations around family caregiver involvement is of great importance when considering nurse's and patient's experience in the dementia context. Family caregivers should be briefly educated on the importance of their presence. This is something that can be expressed to family members at the point of triage. If feasible, advertisement can be instituted in a simple manner about the value of family caregiver involvement while their loved ones are in the ED.

In adopting some of these practice changes a shift in the culture of the ED can occur. If an organization invests in implementing policies and practice changes that improve the experience of nurses and those of patients then already a more person-centred model is instituted. It is not meant to eliminate the current biomedical model of curing a patient, rather attempts at integrating a more holistic model of care can help strengthen the organization and the ED, and subsequently enhance the nurse’s experiences and patient outcomes.

\section{Conclusion}

This study contributes to our knowledge and understanding of the experiences of ED nurses who care for acutely ill elderly patients with an underlying diagnosis of dementia. Findings of this study suggest that the ED culture and dominant practices, a nurse's affinity for acute and episodic care, in combination with the limited dementia-specific knowledge that ED nurses hold, collectively contribute to the difficult and challenging experiences of participants. The narratives of participants and narratives of my own experiences illuminate a lack of competence in assessing and instituting person-centred, tailored interventions to manage 
responsive behaviours. Instead of grounding nursing interventions in evidence-based knowledge we instituted non-person-centred interventions such as pharmacological approaches or restraints in order to manage the behaviours of dementia.

Providing person-centred care to the patient with dementia is further compromised in an ED. This is because of time restrictions and pressures to complete tasks quickly as well as the apparent culture of curing that often supersedes caring during patient interactions. This is contributed to by three factors: first, participants in this study were more familiar with how to manage the physical needs of the patient; second, providing social or psychological care required greater investment of time, which in the ED is limited; and third, participants were fueled by the immediate outcomes they saw and measured when providing acute medical care.

In order to prepare nurses for the reality of the ED and to improve the experiences of nurses and their patients several suggestions have been proposed. First, nurses should be better prepared and oriented to the realities of working in an ED. Second, providing care to patients with dementia requires specialized training and skills, which should be ongoing, in this way care is more holistic and person-centred. Third, nurses and patients can benefit from having available family caregivers, a nurses aid and/or a geriatric nurse specialist available to mitigate some of the challenges associated with not having enough time or knowledge resources. I conclude this study by emphasizing the value of listening to nurses' stories and using their experiences as a catalyst to improve healthcare practice by challenging the status quo and existing socio-political structures that are deeply rooted in the way we currently provide care. Efforts to shift the current ED culture from a predominantly medical model focus to a more synergistic holistic model that values person-centred care will permit nurses and their patients with dementia to have more positive experiences 


\section{CHAPTER 8}

EPILOGUE

MY KNOWLEDGE INFORMED PRACTICE 


\section{Epilogue}

It is the 6th of June, 2013. I have been an ED nurse for five years. I am confident, knowledgeable, and skillful. These days, in addition to my role as an ED nurse I also work as a Geriatric Emergency Management (GEM) nurse. In this role I am a resource to the geriatric patients in the ED and also to my fellow nursing and physician colleagues. I provide appropriate assessments and consultation to address acute symptoms, underlying health conditions, physical, functional, emotional and cognitive status, for at-risk elderly identified in the ED. I have been appointed into this role because of my passion for geriatrics. I have not taken any special courses to prepare me for this role. My passion drove me to educate myself on the topic of geriatrics. It was my experience with Ms. Levine and subsequent similar cases that encouraged me to expand my skill-set in the ED in hopes of making a difference through educating my peers and implementing strategies to improve the lives of patients, in particular those with dementia in the ED. If I could positively influence the experience of even one patient with dementia in the ED and one nurse who looks after them then that to me is grand. I now want to share a more positive story with you, one that has been influenced by my new knowledge of dementia and resources available to me.

\section{Mr. Singh}

I arrive at work in my usual fashion. I enter the ED through the main entrance. I walk through a short hallway where patients are in rooms with curtains to make my way to my office at the end of the hall, where likely I will find a pile of work on my desk waiting for my attention. On this particular day as I passed room number one, I couldn’t help but notice an older patient in posey restraints, tied up to the metal frame of the stretcher. He was quiet but his body restless, his arms tightly wrapped in restraints and at his side, he was constantly squirming and fidgeting 
to be freed. I immediately felt a sense of responsibility. I could not ignore what I was seeing. The patient was barely covered in a blanket, his arms tied to the rails and exposed for anyone who walked by to see him. Flashbacks of Ms. Levine played in my head as I walked to the nursing station to speak with the patient's nurse. I was not surprised to see it was one of our new hired nurses who resorted to restraints and left the patient on display for anyone who entered the ED to see. In speaking with the nurse I got a report that the patient was "very combative last night” and “they had to give him Haldol.” Haldol is a typical antipsychotic drug used in the ED to reduce agitation and aggression in patients with dementia. My immediate reaction was frustration, but I had to understand that this nurse, much like me when I first started, is not knowledgeable at this time, and is likely basing his interventions on his experiential knowledge. Instead of further questioning the nurse I decided to provide him with support around how to avoid using restraints and the risk of having the patient in restraints for a long period of time, and alternative methods of managing the so called combative behaviour. Sometimes, I believe the best lessons are taught through setting good examples.

\section{The Patient's Needs}

I first reviewed Mr. Singh’s history and not surprisingly found that he was from a Long Term Care facility and had a history of dementia. I then assessed his physical status including his medication regimen and any outstanding physician order that had to be processed. Mr. Singh was not ordered an intravenous since his arrival in the ED and also had not been provided any food while in the ED. Likely he was dehydrated and hungry, contributing to his behaviours the previous night. He had a Foley catheter draining his urine, which was an unnecessary intervention and likely used as a measure to save the nurse from having to change the patient frequently for incontinence. This likely contributed to Mr. Singh’s agitation as well. Mr. Singh 
was in an unfamiliar environment, away from his regular caregivers and routine activities, also contributing to his behaviours the night before. Last, being strapped to the stretcher all night, prevented Mr. Singh's ability to move and turn or have control over his body while also limiting mental stimulation; these would also contribute to agitation and boredom.

\section{Meaningful Interventions}

I began by removing Mr. Singh’s posey restraints. The skin around his wrists was irritated and red from all the pulling and tugging he did while trying to free himself. I opened each knot with pleasure and gently rubbed his skin and massaged his wrists to return blood flow back to his hands. I got him up from bed into a sitting position on the stretcher. Mr. Singh was not able to express himself verbally, but the expression of relief and gratitude on his face was evident. I was warned not to get him out of the stretcher because he was unstable on his feet; however, knowing that a proper assessment may not have been done I decide to stand Mr. Singh up anyway. He was shaky, but managed to use a walker to maintain his balance. I tied the back of his hospital gown that was undone. His back exposed, was red, and imprinted with lines from the folds of the linen that were underneath him all night.

As the GEM nurse I had time to attend to the various needs of Mr. Singh. I walked with Mr. Singh for a few seconds but he seemed quite tired and shaky, so after just a few steps I asked him if he wanted to sit in a chair. I brought a reclining chair for him and padded it with blankets to ensure his skin integrity would no longer be compromised. I could see he was already a lot more comfortable. I spoke to Mr. Singh and involved him in everything I would do. I brought him a breakfast tray and assisted him to eat. One thing he did have was a great appetite. I made him a tea and brought the morning paper and asked him if he would like me to read the paper to him. His affect appeared calm and relieved, and his shakiness improved as I read parts of the 
paper to him. I wanted to keep him engaged and stimulated so I went into my office to collect our Montessori Methods cart. In the cart I had various activities that would be appropriate for those at any stage of dementia. I did not want to provide Mr. Singh with an activity that would not be meaningful to him. So I contacted his Long Term Care facility and spoke with the staff about Mr. Singh's routines, interests, skills and abilities. The nurse over the telephone advised me that he is very confused and typically sits at the table and observes while others partake in activities. With this new knowledge I selected appropriate activities to offer Mr. Singh.

I brought out picture cards first, I demonstrated to Mr. Singh how to match the picture cards to a picture template on top of each other. This activity seemed rather simple; however, Mr. Singh was not able to manipulate the cards. Knowing this, he likely would not be able to participate in any activity that required manual dexterity. I brought out a few books on traveling, gardening, and animals that had large photographs and print. I turned the pages as Mr. Singh gazed at the photos. I felt this activity provided him with sensorial stimulation, giving him something appealing to look at instead of the walls in his room. I was amazed at his engagement as his eyes were set on each photo, I wondered what he was thinking about and if the images were bringing memories of times he may have traveled to any of the destinations in the book. That day I stayed with Mr. Singh for a couple of hours. His mood and behaviour changed from somnolent and bored to stimulated and alert. The nurse looking after him was impressed, surprised, and thankful at the transformation in his behaviour from what he was described from the night before. I was rewarded by the outcome of my interventions. I knew I made a difference for Mr. Singh. I also knew I lead by example by providing a new nurse with evidence of how to improve the care for the patient with dementia, even in circumstances that seem hopeless. 


\section{My Journey as an ED Nurse}

As I reflect on who I was in chapter one of this thesis and who I am today I recognize that my ideas about nursing and practice as a nurse has been shaped by both my professional experiences and knowledge I gained by completing this thesis. Like the participants of this study, I wanted to work in the ED to have exposure to critical patients and situations, where I could use technology and skills to save lives. I admired nurses like Susan, I wanted to do CPR on patients, and I wanted to learn everything about the heart and brain. It is not that I don't enjoy the technical aspects of my job, but, I have learned the importance of appreciating and embracing every facet of what it means to be an ED nurse. Being frequently assigned to look after patients with cognitive decline was the major impetus for my desire to learn more about dementia. In completing this thesis I have become more aware my own experiences while learning from the stories of the participants in this study. I now understand that my experiences were not isolated. After listening to the stories of my participants and dissecting every utterance of their narratives, I realize that we shared many of the same difficulties and challenges when caring for patients with dementia. If I remained stagnant in my career I would continue to relive the experiences I had with Ms. Levine. Since the start of this thesis, I have taken charge of my career by expanding my ED skill to include a GEM role. I have grown as a professional nurse and improved my own practice. I have contributed to the practice development of my colleagues, and provide consultative advice to members of my interprofessional team. I learned that to be a good ED nurse I would have to master not only technical skills but also dementia care skills. Overall, listening to the stories others had to share and concurrently reflecting on my own practice has been an educational, transformational, and emancipating experience. And so, the last image I 
include in my research journey is a re-creation of my image in chapter one. See Image 12.

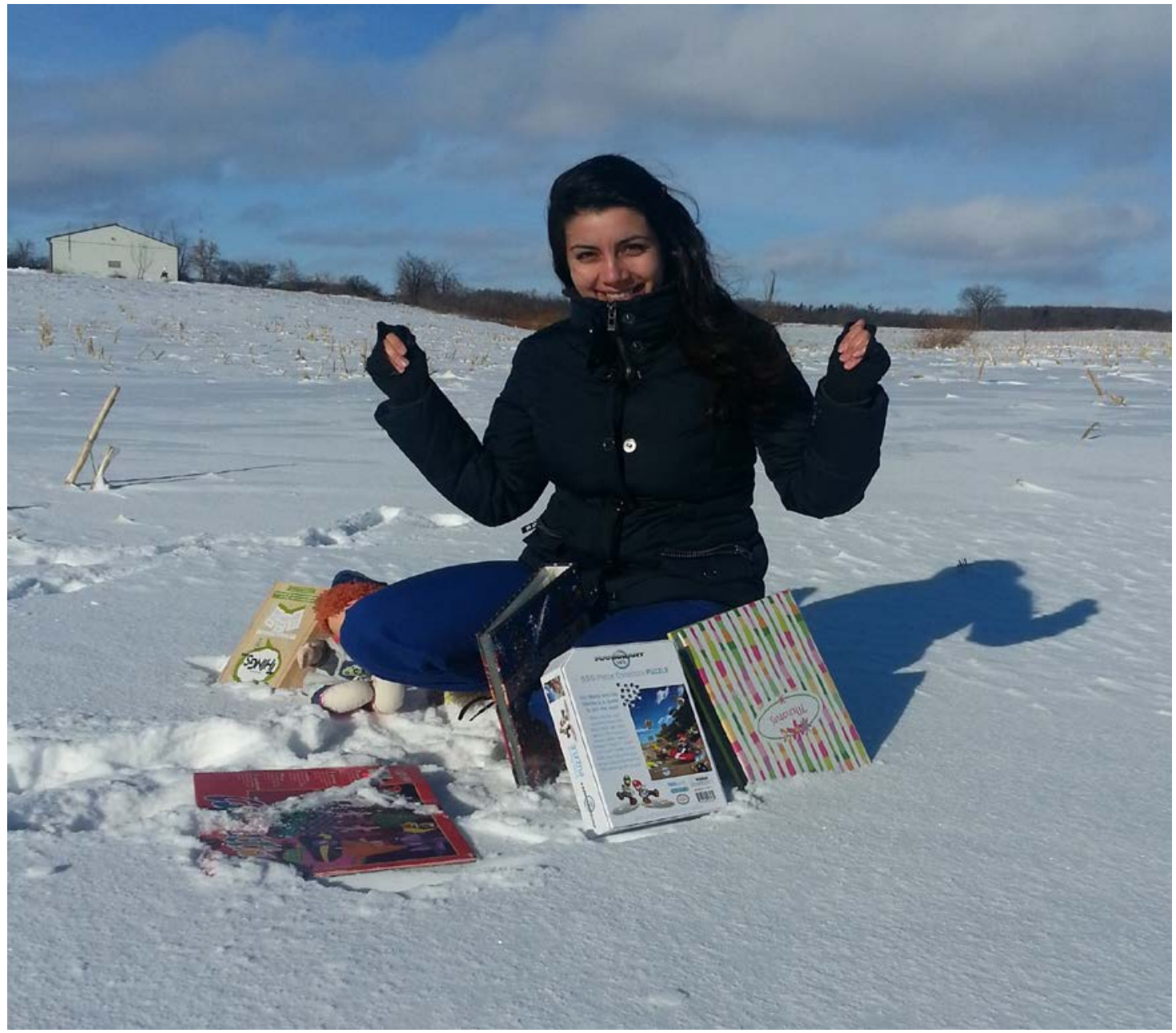

Image 12: New Perspectives

This photograph depicts how the passage of time and the acquisition of knowledge and experience have promoted a positive transformation for me and my nursing career in the ED. I no longer feel alone and helpless. I now have the tools, knowledge and skills to make a meaningful difference in the lives of acutely ill elderly patients with dementia. I have uncovered the dominant discourse and power relations that have affected my practice and care of patients with dementia. It is my hope that my own journey of transformation will be an experience shared by each and every ED nurse. 


\section{APPENDICES}




\section{APPENDIX A: EMERGENCY DEPARTMENT TRENDS}

\section{Emergency Department Trends}

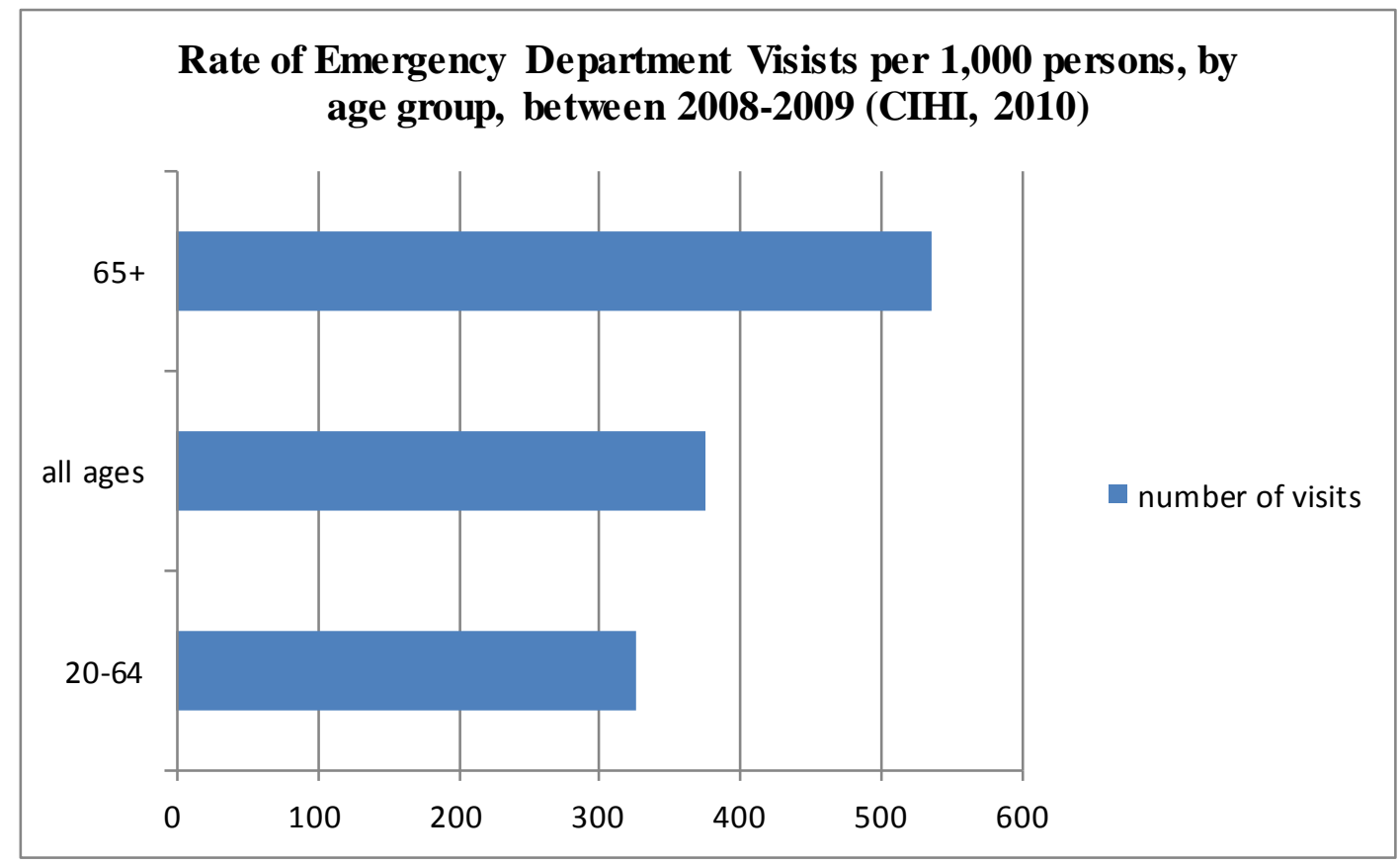




\section{APPENDIX B: HEALTH SERVICE USE TRENDS}

Health service use by Ontario adults aged 66 and older living in the community by presence of physician-diagnosed dementia (ICES, 2011)

\begin{tabular}{|c|c|c|}
\hline \multirow{2}{*}{} & Physician Diagnosed & No Physician Diagnosed \\
& Dementia & Dementia \\
\hline Emergency department visits & $\mathrm{N}=101,775$ & $\mathrm{~N}=1,404,702$ \\
\hline Acute care admissions & $45.9 \%$ & $27.6 \%$ \\
\hline
\end{tabular}




\title{
APPENDIX C: INFORMATION/INVITATION LETTER
}

\author{
RYERSON
UNIVERSITY
Daphne Cockwell
SCHOOL OF NuRSING \\ Information Letter/e-mail - Phase 1 Recruitment \\ Invitation to Participate in a Research Study
}

Dear Potential Participant,

My name is Pearl Shalchi, and I am a Master of Nursing student from Ryerson University. I am writing to ask you for your participation in a research study that is being conducted as part of the requirements for the completion of my Master's degree in nursing at Ryerson University, Daphne Cockwell School of Nursing. This study is intended to increase our understanding of the everyday experiences of emergency nurses who provide care for acutely ill elderly patients with an underlying diagnosis of dementia who present with the responsive/challenging behaviours of dementia such as agitation, aggression and wandering in the emergency department. You have been invited to participate in this study because you are a practicing bedside Emergency Department (ED) nurse.

If you agree, your participation in this study will include:

1. Taking photographs with a disposable camera provided to you of metaphorical images, outside of the workplace off of hospital property, that reflect your feelings and thoughts with respect to caring for older adults living with dementia who present to the ED.

2. Taking part in two face-to-face interviews of approximately 60-90 minutes in length 2-3 weeks apart, that takes place outside of your scheduled work time.

3. Consent to being audio-taped during the two interviews.

If you decide to take part in this study, I will be asking you to sign a letter formally stating your consent to participate. If you are interested in participating or learning more about the study to assist you in reaching decision about participation please contact the principal investigator using the contact information provided below. Thank you for your time and consideration.

Pearl Shalchi, RN

Principal Investigator

MN Student, Ryerson University, Daphne Cockwell School of Nursing

647-654-0970

nshalchi@ryerson.ca

Dr. Lori Schindel Martin, RN, PhD

Thesis Supervisor, Ryerson University, Daphne Cockwell School of Nursing

416-979-5000, ext. 4257

lori.schindelmartin@ryerson.ca 


\section{Recruiting Participants for a Research Study}

\section{Dementia Narratives of Emergency Department Nurses}

\section{Recruiting}

Emergency Nurses who have experienced caring for acutely ill elderly patients with an underlying diagnosis of dementia in the emergency department.

\section{Purpose of Research}

To increase our understanding of the everyday experiences of emergency nurses who provide care for acutely ill elderly patients with an underlying diagnosis of dementia who present with the responsive/challenging behaviours of dementia in the emergency department.

What is involved?

- Two individual interviews that may last from 60-90 minutes at a negotiated time and location

- Taking photographs with of metaphorical objects with a disposable camera provided to you

\section{Compensation}

It is not anticipated that you will incur any cost for participating in this research study

As a token of appreciation for your participation you will receive a \$10 Tim Horton’s gift card

\section{Confidentiality}

Your identity and any information you share will be kept in the strictest confidence. If you decide to take part in this study, I will be asking you to sign a letter formally stating your informed consent to participate.

If you are interested, please contact me:

Pearl Shalchi, RN

Principal Investigator

MN Student, Ryerson University

Daphne Cockwell School of

Nursing

647-654-0970

nshalchi@ryerson.ca
RYERSON

UNIVERSITY

Daphne Cockwell SCHOOL OF NURSING
Thesis Supervisor

Dr. Lori Schindel Martin

RN, PhD

Ryerson University, Daphne

Cockwell School of Nursing 


\section{RYERSON
UNIVERSITY
Daphne Cockwell
school OF NuRsING \\ Information and consent form Dementia Narratives of Emergency Department Nurses}

You are invited to participate in a research study. This study is being conducted as part of the requirements for the completion of my Master's degree in nursing at Ryerson University, Daphne Cockwell School of Nursing. It is important you read this form as it provides information you will need to know in order to decide whether you wish to participate in this study. Before you give your consent to be a participant, it is important that you read the following information provided. Please ask any questions necessary to help you understand what you will be asked to do.

\section{Investigators:}

Principle Investigator: Pearl Shalchi, RN, MN student, Ryerson University, Daphne Cockwell School of Nursing, Faculty of Community Services, Ryerson University, Toronto, Ontario Thesis Supervisor: Dr. Lori Schindel Martin, RN, PhD, Thesis Supervisor, Ryerson University, Daphne Cockwell School of Nursing, Faculty of Community Services, Ryerson University, Toronto, Ontario.

\section{Purpose of the Study:}

Emergency Departments (EDs) serve as the main portal of entry to hospitals for persons requiring health care services (Chan, Schull \& Schultz, 2001). The Emergency Department typically assumes responsibility of older patients with dementia during their most acute stage of illness, such as during an acute stroke, fracture or infection; therefore, placing additional responsibility on ED nurses (Kihlgren et al., 2004). Providing nursing care becomes challenging when nurses have to manage routine emergency nursing care of patients in addition to the physical and responsive behaviours of dementia. The purpose of this study is to understand the everyday experiences of emergency nurses who provide care for acutely ill elderly patients with an underlying diagnosis of dementia who present with challenging behaviour in the emergency department.

\section{Description of the Study:}

Your participation in this study requires you to share your experiences as a nurse in the ED caring for acutely ill elderly persons with dementia. Information you provide will be collected during private and individual interview sessions with the principal investigator at a time and location convenient for you. The interviews will take place during April 2013 through June 2013. There will be two interviews approximately 60-90 minutes in length and one brief 5-10 minute meeting which are all separated by about $2-3$ weeks' time. The interviews will take place outside of your work day at a private location of your choice. With your permission these 
interviews will be tape-recorded, and will be transcribed by the principal investigator following the interview.

\section{If you consent to participate in this study, you will:}

1. Be provided with a disposable camera and instructions to take 6 photographs of real objects or metaphorical images, outside of the workplace and off of hospital property, that reflect your feelings and thoughts with respect to caring for older adults living with dementia who present to the ED. The photographs can be pictures of nature, inanimate objects, animals/pets, foods etc. Photographs will exclude patients, their families, hospital staff including nursing colleagues, or any photos in the workplace or anywhere on hospital property. These illustrations will then be discussed during the second interview and used in conjunction with your interview data to help depict your experiences in both written and visual language.

2. Take part in two face-to-face interviews of approximately $60-90$ minutes in length $2-3$ weeks apart, that take place outside of your scheduled work time.

3. Meet with the principal investigator approximately 2 weeks after the first interview for 510 minutes at a negotiated time and location convenient for you. At this time the disposable camera will be collected so that the photographs can be developed prior to the second interview

4. Consent to being audio-taped during the two interviews. With your permission these interviews will be tape-recorded and transcribed following the interview.

\section{The first interview:}

Upon your consent, the first interview will take place at a comfortable, convenient and private location that you have chosen. This interview will be audio-taped. I will also take notes during this interview to ensure I have documented all the experiences you have shared with me. You will be given the opportunity to share your experiences of caring for elderly patients who have dementia. The length of this interview depends on how you are feeling at the time. It will be approximately 60-90 minutes; you can choose to end it at any time. A short demographic questionnaire will be given to you to complete during this interview as well. At the end of the first interview I will provide you with a disposable camera and verbal and written instructions on this activity. At this time if you wish to continue participation in this study we will schedule a 510 minute meeting at a time and location convenient for you to collect the camera.

\section{Meeting:}

Approximately 2 weeks after our first meeting we will see each other again for approximately 510 minutes so that the camera can be collected and photographs printed in time for the second interview. At this time if you wish to continue participation in this study we will schedule the second interview in approximately 2-3 weeks at a time and location convenient for you. This second interview will be approximately 60-90 minutes in length. 


\section{The second interview:}

The second interview will take place approximately 2-3 weeks after the first interview. Again, this will be arranged at a location and time that is convenient for you. During the second interview you will be given an opportunity to share any further information you may have not remembered in the first interview. I will also have a chance to ask you clarification questions or to elaborate on interview data from our first interview. At this time we will review the pictures you have taken with the disposable camera provided to you during recruitment. You will be given an opportunity to share your reflections and thoughts on the meanings the photos have for you. With your consent, these photographs will be included in their original form in the final research report in a collage format. These photographs may be used for presentation and publication purposes, but your identity will always remain anonymous.

\section{What is Experimental in this Study?}

This study is not experimental in nature. The only experimental aspect of this study is the gathering of information for the purpose of analysis.

\section{Risks or Discomforts:}

It is not anticipated that participants will experience discomfort or distress from participation in this study. You may remember some unpleasant memories about your experiences with providing care for persons with dementia while you are participating in interviews. At any point if you feel uncomfortable, you can discontinue your participation in the study either temporarily or permanently. If at any time during your participation in this study you feel distressed or sad and want to speak with someone you will be provided with an information brochure on your institutions' affiliated employee assistance program which includes services such as counselling.

\section{Benefits of the Study:}

There will be no direct benefits to you from participation in this study. It may be interesting to you to discuss your experiences with me, and this may represent some benefit to you. Your responses will help us better understand the experiences of emergency nurses who provide care for acutely ill elderly persons with dementia.

\section{Confidentiality:}

Any information obtained in connection with this study will remain confidential. No identifying information about you or that of the hospital you work for will appear in any report of the research. In interview data and research reports you will be referred to using a Pseudonym of your choice. Interview data you provide will be kept in a locked filing cabinet in the principal investigators home office. During the interviews, you do not have to provide any information that will identify you. The researcher will keep what you share in the interviews strictly confidential.

During the interviews what you share will be documented through note taking as well as tape recorded. This will ensure that I do not miss any of your valuable experiences shared. When I turn the interview tape recordings into writing, I will remove all the information that identifies you. I will store all media files on an encrypted password protected USB stick and place this and paper records in a locked cabinet in the principal investigators home office in a locked filing cabinet for a duration of 2 years after the study has been completed. After which they will be 
both deleted of the media devices and paper data will be destroyed and disposed of in a confidential manner. All transcript data will be kept in a personal password protected computer which be located in the principal investigators home office in a locked filing cabinet. The study reports will never show your name or any information that identifies you or the hospital for which you work.

Your co-workers or any member of your management team will not be able to identify you with any research data. All data including visual and interview information you provide will not be used for any purpose other than this study. Full transcripts will only be seen by my immediate supervisor, Dr. Lori Schindel Martin. No other thesis committee members will see anything other than aggregate data (the data in a report form) that will not have your name or any identifying information attached. Some of your comments from the interviews may be used word-for-word to present results of the study, but no one will know that you were the person who said those words. The photographs that you provide will be used in their original form for the study and in the final research report, but your identity will remain anonymous. If you prefer that photographs are not used in the final research report, that will be respected.

\title{
Incentives to Participate:
}

As a token of appreciation for your participation you will receive a \$10 Tim Horton’s gift card.

\section{Cost and/or Compensation for Participation:}

It is not anticipated that you will incur any personal cost for participating in this research study

\section{Voluntary Nature of Participation:}

Your participation in the study is completely voluntary and you may choose to stop participating at any time without penalty. At any point in the study, you may refuse to answer any particular question or stop participation altogether. You can request that audio-taping be stopped at any time. Your decision not to participate will not influence the nature of your relationship with your employer or Ryerson University now, or in the future.

\section{Questions about the Study:}

If you have any questions or concerns about the study now or at any point during or after the interviews, you may contact:

\author{
Pearl Shalchi (Researcher, MN student) \\ nshalchi@ryerson.ca \\ 647-654-0970 \\ Or \\ Dr. Lori Schindel Martin (Thesis Supervisor) \\ 416-979-5000, ext. 4257 \\ lori.schindelmartin@ryerson.ca
}

This study has been reviewed by the Research Ethics Board at XXXX and the Ryerson University Research Ethics Board (REB). If you have questions regarding your rights as a human 
subject and participant in this study, you may contact any of the following research ethics boards for information.

\author{
Ryerson University Research Ethics Board \\ Research Ethics Board \\ C/o Office of the Vice President, Research and Innovation \\ Ryerson University \\ 350 Victoria Street \\ Toronto ON M5B 2K3
}

$\mathrm{T}:$ 416-979-5042

OR

XXXX

\title{
Consent:
}

My signature below indicates that I have read the information in this agreement and have had a chance to ask any questions I have about the study. I agree to be in the study and have been told that I can change my mind and withdraw my consent to participate in this study at any time. I understand and agree that the photographs I take of objects during the study will be used for presentation and dissemination purposes, but that my identity will remain anonymous. I understand that the photos I will be taking as part of this study are of metaphorical images only. I understand and agree that photographs of any patients, their family members or hospital staff that I work with are not to be taken as part of this study. I understand and agree that I will not take photographs in my workplace or anywhere on hospital property. I understand and give my permission for the interviews to be audio-taped. I understand that I may ask audio-taping be stopped at any point during the interviews. I have been given a copy of this agreement. I have been told that by signing this consent agreement I am not giving up any of my legal rights.

Participants Name (please print) _ , Toronto, Ontario

Participants Signature Date

Name of Investigator

Signature of Investigator

Date 


\section{APPENDIX F: INTERVIEW \#1 GUIDE}

\section{Interview Guide One}

\section{Introduction:}

Hi, my name is Pearl Shalchi, and I am a Master of Nursing student at Ryerson University. I am doing this study as part of the requirements for my program completion. The inspiration for this study grew from my own personal experiences as an ED RN. Your participation in this study will help us gain a better understanding of the everyday experiences of emergency nurses who provide care for acutely ill elderly patients with an underlying diagnosis of dementia who present with challenging behaviour in the emergency department.

\section{Open-ended introductory questions:}

1. Tell me about what you did before you became a nurse (i.e., what kind of work)

2. Why did you choose to be a nurse? (i.e., what was the appeal to you?)

3. What was nursing school like for you? Can you describe your academic experience? (i.e., favorite courses, most challenging courses, friends, teachers, etc.?

4. What motivated you to work in the ED? (i.e., peers, shiftwork, challenge, convenience?)

5. What assumptions did you have about the ED prior to working there?

6. Was there a time in your ED career where you felt you either appreciated and wanted to stay in your job or wanted to leave your job and work in a different area?

- What experience led you to feel this way, who was involved, was it preventable? Did something or someone upset you, what made you stay?

7. What is it like to care for elderly patients in the ED? (i.e., easy, rewarding, challenging, complex, etc.?), can you give me specific examples?

8. Can you tell me what it is like to care for elderly patients with dementia in the ED?

- Do you remember a particular patient with dementia or an incident (either positive or negative) you can share with regards to caring for this patient in the ED

- Is there more than one experience that has resonated with you that you can share?

- What was the context of the event(s)? What behaviours did the patient display? Who was there, what time of day was it, were you alone? How did you feel, how did the patient feel? What effect has that situation(s) had on you since? What strategies did you use? What worked and what didn't work

- What was your knowledge about dementia prior to caring for this patient(s)

- What were/are your assumptions about dementia?

- Did you feel adequately prepared to care for those patients at that time, how about now? What are some barriers to care? What strategies would you use next time?

- How has the environment in which you work influenced your experience

\section{Probing questions:}

1. Can you explain what you mean when you say...

2. Can you tell me more about. . .

3. Can you expand a little more about. . .

\section{Closing:}

Is there anything more you would like to add? Do you have any questions? 


\section{APPENDIX G: INTERVIEW \#2 GUIDE}

\section{Interview Guide Two}

\section{Introduction:}

A few weeks ago I asked you a series of questions about your experiences. We are meeting again today to give both of us an opportunity to reflect back on that meeting and ask any further questions or clarify previous responses. This meeting will also allow us to review and reflect on the photographs you have taken to describe your experiences of caring for older persons with dementia in the ED.

\section{Open-ended introductory question:}

1. Is there anything in general that you want to share with me today that you did not have an opportunity to do so in the last meeting?

2. Looking at these photographs you have taken, which one would you say is your favorite and why?

3. Which one of these photos best represents your experiences of caring for older persons with dementia in the ED?

4. What meaning does this photograph have for you?

5. Why did you choose to take this photograph? What elements of this photo are important to you?

6. Would you change anything about this photograph?

7. How did you feel emotionally, physically or otherwise while you were taking these photographs?

8. Did you gain any benefits from this activity?

9. Was this activity challenging for you? If so, can you describe this?

\section{Probing questions:}

In the last meeting you talked about. . . , can you elaborate on. . .

Can you expand a little more on. . .

Can you explain what you meant when you said. . .

\section{Closing:}

Is there anything more about your experience that you would like to add? Do you have any questions or final comments? Thank you for your participation and time. 


\section{APPENDIX H: PHOTOGRAPHY ACTIVITY INFORMATION SHEET}

\section{Photography Activity Information Sheet}

\section{Dear Participant,}

In research studies there are a variety of ways to collect data and photography is one creative method in doing so. Using the disposable camera provided to you, you are asked to take pictures of metaphorical objects such as nature, inanimate objects, pets/animals, or foods meant to be representative of your experiences in caring for persons with dementia in the ED. This will exclude taking pictures of patients, their families, nursing colleagues, or any images of the hospital property and your workplace. These photos should reflect the feelings, values, and ideas of your experiences caring for older people with dementia in the ED.

The photography you provide will add richness and depth to the stories you share in the interview. Essentially, photographs will embody your lived experience and feelings. The 6 photographs you choose to take will be used in conjunction with the verbal accounts you share to enhance and provide further insight into the meaning of your experiences. Photographs will be used in their original form as part of the research study and included as a collage in the final research report. Photographs may also be used for publication or presentation purposes. If you prefer that photos are not used in reports, publications or presentations, I will respect that. You may be as creative as you like with your photography. The objective is to reflect on your experiences and capture them in photographs. We will schedule a brief meeting after the first interview to pick up the camera in order for the photographs to be developed.

The photographs you take will be printed in 2 copies; you will be given a copy of all the photographs to keep. During the second interview we will discuss and reflect upon the photographs and explore their meaning to you and how they are representative of your experiences in caring for persons with dementia in the ED.

If you have any questions about this creative activity, please do not hesitate to ask me at any time during the study.

Thank you,

Pearl Shalchi, RN

Principal Investigator

MN Student, Ryerson University, Daphne Cockwell School of Nursing

647-654-0970

nshalchi@ryerson. 


\section{APPENDIX I: CAMERA COLLECTION MEETING GUIDE \\ Camera Collection Meeting Guide}

\section{Introduction:}

In our last meeting I provided you with a disposable camera to take photographs that reflect your feelings and thoughts related to your experiences with caring for acute ill elderly patients with an underlying diagnosis of dementia. We are meeting to so that I can collect the disposable camera at this time. I will print 2 sets of the photos you have taken and ensure that you receive a copy of these on a media disc as well as hard copy.

\section{Questions:}

1. Do you have any questions at this time related to the photography activity you have participated in?

2. Do you have any questions, comments or concerns related to any part of this research study so far?

3. In approximately 2-3 weeks I would like to meet with you again for a second interview that will take 60-90 minutes. At this time we will both have an opportunity to ask questions as well as clarify anything from our first interview.

4. During the second interview we will spend time reflecting upon and talking about the photos you have taken.

5. Would you like to proceed to a second interview? If not, thank you very much for participating in this study, if you would like a copy of your photographs please provide me with where you would like this sent

6. If you would like to meet for the second interview, when is a good date and time for me to meet with you again and at what location?

Thank-you for meeting with me today. 


\section{References}

Alzheimer Society. (2013). Shifting focus: a guide to understanding unpredictable dementia behaviour. Retrieved from http://www.alzheimer.ca/en/About-dementia/Understandingbehaviour/guide-to-dementia-behaviour

Alzheimer Society. (2012). Person-centred language. Alzheimer Society of Ontario. Retrieved from http://www.alzheimer.ca/ /media/Files/national/Culturechange/culture_person_centred_language_2012_e.ashx

Alzheimer's Society of Canada. (2012). Culture change towards person-centred care. Retrieved from http://www.alzheimer.ca/en/Living-with-dementia/Caring-for-someone/Long-termcare/culture-change-person-centred-care

Alzheimer’s Society of Ontario. (2012). Dementia evidence brief: North Simcoe Muskoka Local Health Integration. Retrieved from http://www.alzheimer.ca/guelph/ /media/Files/on/PPPI\%20Documents/ASO-DementiaEvidence-Brief/ASO\%20Dementia\%20Evidence\%20Brief\%20-\%20NSM.ashx

Alzheimer’s Society. (2011). Guidelines for care: person-centred care of people with dementia living in care homes framework. Retrieved from http://www.alzheimer.ca/ /media/Files/national/Culturechange/culture_change_framewo $\underline{\text { rk_e.ashx }}$

Alzheimer Society. (2010). Rising tide: the impact of dementia on Canadian society. (2010). Alzheimer Society of Canada. Retrieved from http://alzheimersociety.sitesystems.ca/sitecore/shell/Controls/Rich\%20Text\%20Editor/ / media/Files/national/pdfs/English/Advocacy/ASC_Rising\%20TideExecutive\%20Summary_Eng.ashx 
Andrews, J., \& Christie, J. (2009). Emergency care for people with dementia. Emergency Nurse 17(5), 12-15.

Benner, P. (1984). From novice to expert: excellence and power in clinical nursing practice. Addison-Wesley: Menlo Park.

Bianchetti, A., \& Trabucchi, M. (2004). Behavioural and psychological symptoms of dementia: clinical aspects. Neuroscience Research Communications, 35(3), 173-183.

Blauner, B. (1987). Problems of editing “first person” sociology. Qualitative Sociology, 10, 4664.

Borbasi, S., Jones, J., Lockwood, C., \& Emden, C. (2006). Health professionals perspectives of providing care to people with dementia in the acute care setting: toward better practice. Geriatric Nursing 27(5), 300-308.

Brown, J.A. (2000). The potential contributions of critical social theory to nursing science. Canadian Journal of Nursing Research 32(2), 35-55

Bruce, M.E. (2002). Narrative research: a spiritual and liberating approach to research. Religious Education 103(3), 323-338

Burgess, L., Page, S. (2003). Educating nursing staff involved in the provision of dementia care. Clinical Research and Development 99(46), 34-37

Burnard, P. (1991). A method of analyzing interview transcripts in qualitative research. New Education Today 11, 461-466.

Canadian Institute for Health Information (2010). Seniors' use of emergency departments in Ontario, 2004-2005 to 2008-2009. Retrieved from http://secure.cihi.ca/cihiweb/products/seniors_ed_e.pdf

Canadian Mental Health Association (n.d.). Seniors and mental health. Retrieved from 


\section{http://www.ontario.cmha.ca/seniors.asp}

Carr, M.F. (2013). The Role of Sitters in Delirium: an Update. Canadian Geriatrics Journal, $16(1), 22-36$

Cerejeira, J., Lagarto, L., \& Mukaetova-Ladinska, E.B. (2012). Behavioral and psychological symptoms of dementia. Frontiers in Neurology, 3(73), 1-21.

Chan, T.B., Schull, J.M., Schultz, S. (2001). Emergency department dervices in Ontario 19932000. Institute for Clinical Evaluative Sciences. Retrieved from $\underline{\text { http://www.ices.on.ca/file/Emergency_department_services_in_Ontario.pdf }}$

Chang, E., Daly, J., Johnson, A., Harrison, K., Easterbrook, S., Bidewell, J., . . Hancock, K. (2009). Challenges for professional care of advanced dementia. International Journal of Nursing Practice, 15(1), 41-47.

Cipriani, G., Vedovello, M., Nuti, A., \& Di Fiorino, M. (2011). Aggressive behavior in patients with dementia: Correlates and management. Geriatrics \& Gerontology International, 11(4), 408-413.

Clandinin, D.J. (2007). Handbook of narrative research: mapping a methodology. Thousand Oaks CA: Sage

Clandinin, D. J., \& Connelly, F. M. (2000). Narrative research: experience in story in qualitative research. San Francisco: Jossey-BassCreswell J. W. (2007). Qualitative inquiry and research design: Choosing among five approaches (second ed.). Thousand Oaks CA: Sage

Claudia, K.Y., \& Arthur, D. (2003). Wandering behaviour in people with dementia. Journal of Advanced Nursing 44(2), 173-182. 
Cohen-Mansfield J, Libin A, Marx MS. (2007). Nonpharmacological treatment of agitation: a controlled trial of systematic individualized intervention. Journal of Gerontology: Medical sciences 62: 908-916.

Cohen-Mansfield J, \& Werner P. (1997). Management of verbally disruptive behaviours in nursing home residents, The Journals of Gerontology 52, 369-377.

Colombo M., Vitali S., Cairati M., Perelli-Cippo R., Bessi O., Gioia P. \& Guaita A. (2001) Wanderers: features, findings, issues. Archives of Gerontology and Geriatrics, 33, 99-106

Cowdell, F. (2010). The care of older people with dementia in acute hospitals. International Journal of Older People Nursing 5(2), 83-92.

Crabtree, J., Mack, J. (2010). Designing a training program to improve staff attitudes towards people with dementia. Nursing Times 106

Creswell, J. W. (2007). Qualitative inquiry and research design: choosing among five traditions (second ed.). Thousand Oaks CA: Sage

Cunningham, C. (2006). Understanding challenging behaviour in patients with dementia. Nursing Standard 20(47), 42-45.

Cunningham, C., Archibald, C. (2006). Supporting people with dementia in acute hospital settings. Nursing Standard 20(43), 51-55.

Dewing, J. (2005). Screening for wandering among older persons with dementia. Nursing Older People 17(3), 20-24.

Duffy, M. (2007). Narrative research: The Method. In P.L. Munhall (Ed.), Nursing Research A Qualitative Perspective. (pp. 403-419). Mississauga: Jones and Bartlett

Dupuis, L.S., Wiersma, E., Loiselle, L. (2012). Pathologizing behavior: meanings of behaviors in dementia care. Journal of Aging Studies 26, 162-173. 
Elliot, G. (2013). Montessori methods for dementia: find the story behind the dementia. Handout for Montessori methods for dementia. DementiaAbility Enterprise: Oakville Emergency Nurses Association. (1999). Scope of emergency nursing practice. Retrieved from http://www.coloradonursingcenter.org/sites/default/files/Scope\%20of\%20Practice\%20E mergency\%20Nurses\%20Association\%201999.pdf

Eriksson, C., \& Saveman, B. (2002). Nurses' experiences of abusive/non-abusive caring for demented patients in acute care settings. Scandinavian Journal of Caring Sciences, 16

Fessey, V. (2007). Patients who present with dementia: exploring the knowledge of hospital nurses. Nursing Older People, 19(10), 29.

Finkel S.I, Costa e Silva J., Cohen G., Miller S., Sartorius N. (1996). Behavioral and psychological signs and symptoms of dementia: a consensus statement on current knowledge and implications for research and treatment. International Psychogeriatrics 8(3), 497-500

Gililland, M. (2010). Dementia. Journal of Practical Nursing, 60(1), 9-15.

Hall G., Buckwalter, K. (1987). Progressively lowered stress threshold : A conceptual model for care of adults with Alzheimer's. Archives of Psychiatric Nursing 1, 399-406

Huabin, L., Lin, M., Castle, N. (2011). Physical restraint use and falls in Nursing Homes: a comparison between residents with and without dementia. American Journal of Alzheimer's Disease and Other Dementias 26(1), 44-50.

Hoe, J., Thompson, R. (2010). Promoting positive approaches to dementia care in nursing. Nursing Standard 25(4), 47-56.

Holloway, I., \& Freshwater, D. (2007). Narrative research in nursing. Blackwell Publishing: Oxford, UK. 
Hwang, U., \& Morrison, R. S. (2007). The geriatric emergency department. Journal of the American Geriatrics Society, 55(11), 1873-1876.

Inouye, S., Bogardus S., Charpentier, P., Leo-Summers L., Cooney, L. (2000). The Hospital Elder Life Program: a model of care to prevent cognitive and functional decline in older hospitalized patients. Journal of the American Geriatric Society 48, 1697-1076.

Institute for Clinical Evaluative Sciences. (2011). Health system use by frail Ontario seniors. Retrieved from http://www.ices.on.ca/file/ICES_AgingReport_2011.pdf

Jakobsen, R., Sorlie, V. (2010). Dignity of older people in a nursing home: narratives of care providers. Nursing Ethics, 17(3), 289-300.

Kelly T., \& Linsey H. (2007). Working with stories in nursing research: procedures used in narrative analysis. International Journal of Mental Health Nursing 16, 136-144

Kihlgren, L.A., Nilsson, M., Sorlie, V. (2004). Caring for older patients at an emergency department-emergency nurses’ reasoning. Journal of Clinical Nursing, 14, 601-608

Kihlgren, L.A., Nilsson, M., Skovdahl, K., Palmblad, B., Wimo, A. (2004). Older patients awaiting emergency department treatment. Scandinavian Journal Caring Science 18, 169-176.

Kitwood, T. (1997). Dementia reconsidered: The person comes first. Buckingham: Open University Press.

Kucera, K., Higgins, I., McMillan, M. (2010). Advanced nursing practice: a futures model derived from narrative analysis of nurses' stories. Australian Journal of Advanced Nursing 27(4), 43-53.

Lai, C. K. Y., \& Arthur, D. G. (2003). Wandering behaviour in people with dementia. Journal of Advanced Nursing, 44(2), 173-182. 
Lejman, E., Westerbotn, M., Poder, U., Wadensten, B. (2013). The ethics of coercive treatment of people with dementia. Nursing Ethics 20(3), 248-262.

Lieblich, Amia, Rivka Tuval-Mashiach and Tamar Zilber (1998). Narrative research. reading, analysis and interpretation. Thousand Oaks, London and New Delhi.

LHINS Alzheimer’s Society of Ontario. (2007). Projected prevalence of dementia:

Ontario's Local Health Integration Networks. Retrieved from: http://www.alzheimer.ca/on/ /media/Files/on/PPPI\%20Documents/Projected-Prevalenceof-Dementia-LHINs-April-2007.ashx

MAREP \& Alzheimer Society of Hamilton \& Halton, Brant, Haldimand-Norfolk, \& Niagara Region. (2005). Allcroft, K., \& Loiselle, L., editors. Managing and accommodating responsive behaviours in dementia care: A resource guide for Long-Term Care. Waterloo: University of Waterloo

Martin, B. (2002). Restraint use in acute and critical care settings: changing practice. AACN Clinical Issues 13(2), 294-306.

McCloskey, M. R (2004). Caring for patients with dementia in an acute care environment. Geriatric Nursing 25(3), 139-144.

McCormack, B,. \& McCance, T.(2010). Person-centred nursing : theory and practice. Wiley Publishing.

Ministry of Health and Long Term Care. (2006). Improving access to emergency care: addressing system issues. Toronto: Queen's Printer of Ontario.

Moyle, W., Olorenshaw, R., Wallis, M., \& Borbasi, S. (2008). Best Practice for the management of older people with dementia in the acute care setting: a review of the literature. International Journal of Older People Nursing 3, 121-130. 
Nelson, D., \& Hillan, E. (1995). Student nurses perception of accident and emergency nursing: do they match the reality? Accident and Emergency Nursing, 3, 142-145.

Nolan, L. (2006). Caring connections with older persons with dementia in an acute hospital setting - a hermeneutic interpretation of the staff nurse's experience. International Journal of Older People Nursing 1, 208-215

Nystrom, M., Dahlberg, K., \& Carlsson, G. (2003). Non-caring encounters at a emergency care unit-a life-world hermeneutic analysis of an efficiency-driven organization. International Journal of Nursing Studies, 40, 761-769

Patterson, B., Bayley, E.W., Burnell. K., \& Rhoads, J. (2009). Orientation to emergency nursing, perceptions of new graduate nurses. Journal of Emergency Nursing, 36(3), 203-211.

Person, J., Spiva, L., \& Hart, P. (2012). The culture of an emergency department: an ethnographic study. International Emergency Nursing 21, 222-227.

Purandare, N., Burns, A. (2000). Behavioural and psychological symptoms of dementia. Clinical Gerontology 10, 245-260.

Registered Nurses Association of Ontario. (2003). Screening for delirium, dementia and depression in older adults. Toronto Ontario: Registered Nurses' Association of Ontario.

Richards, E. (2011). Delirium. Nursing Standard, 26(9), 59-60

Ryan, D., Liu, B., Awad, M., Wong, K. (2011). Improving older patients' experience in the emergency room: the senior friendly emergency room. Aging Health 7(6), 901-909.

Sander, R. (2002). Standing and moving: helping people with vascular dementia. Nursing Older People, 14(1), 20-26.

Sandhaus, S., Zalon, L.M., Valenti, D., Dzielak, E., Smego, A.R., Arzamasova, U. (2010). A volunteer-based hospital elder life program to reduce delirium. The Health Care 
Manager, 29(2), 150-156.

Shapiro, B., \& Mervis, J.R. (2007). Distinguishing delirium and dementia. Aging Health, 3(1), 33-48.

Sourial, R., McCusker, J., Cole, M., \& Abrahamowicz, M. (2001). Agitation in demented patients in can acute care hospital: prevalence, disruptiveness, and staff burden. International Psychogeriatrics, 13(2), 183-197

Speziale, H. J. S., \& Carpenter, D. R. (2007). Qualitative research in nursing: advancing the humanistic imperative. (4 ${ }^{\text {th }}$ ed.). Philadelphia: Lippincott, Williams \& Wilkins.

Smith, M., Hall, R.G., Gerdner, L., Buckwalter, C.K. (2006). Application of the Progressively Lowered Stress Threshold model across the continuum of care. Nursing Clinics of North America, 41, 57-81

Sumner, J. (2010). Reflection and moral maturity in a nurse’s caring practice: a critical perspective. Nursing Philosophy, 11(3), 159-169.

Swickhamer, C., Colvig, C., \& Chan, S.B. (2013). Restraint use in the elderly emergency department. The Journal of Emergency Medicine 44(4), 869-874.

Tolson, D., Smith, M., \& Knight, P. (1999). An investigation of the components of best nursing practice in the care of acutely ill hospitalized older patients with coincidental dementia: a multi-method design. Journal of Advanced Nursing 30(5), 1127-1136.

Travers, M.C., Beattie, E., Martin-Khan, M., \& Fielding, E. (2013). A survey of the Queensland healthcare workforce: attitudes towards dementia care and training. BMC Geriatrics 13(101), 1-7.

Wade, T.D., \& Halligan, P.W. (2004). Do biomedical models of illness make for good healthcare systems? BMJ 329, 1398-1401 
Warchol, K. (2006). Facilitating functional and quality-of-life potential: strength-based assessment and treatment for all stages of dementia. Topics in Geriatric Rehabilitation, 22(3), 213-227.

Weirather, R.R. (2010). Communication strategies to assist comprehension in dementia. Hawaii Medical Journal 69, 72-74.

Weitzel, T., Robinson, S., Barnes, R.M., Berry, T., Holmes, J., Mercer, S.,... Kirkbride, G. (2011). The special needs of the hospitalized patient with dementia. Medsurg Nursing 20(1), 13-19. 


\section{GLOSSARY}

Agitation: Agitation is a broad concept often used interchangeably with aggression in dementia related literature (Purandare \& Burns, 2000). Agitation is a responsive behaviour associated with verbal, vocal, or motor activity common in individuals with dementia and can be divided into four subtypes.

Behavioural and psychological symptoms of dementia : A diverse group of symptoms and behaviors which occur in people with dementia (Cerejeira Lagarto, \& Mukaetova-Ladinska, 2012) causing disturbances in perception, thought content, mood, motor activity, altered personality traits, and behaviour (Cerejeira, et al., Bianchetti \& Trabucchi, 2004; Finkel, Costa e Silva, Cohen, Miller, \& Sartorius, 1996).

Confusion Assessment Method Instrument (CAM): A tool developed to distinguish the presence of delirium from other cognitive impairments. This instrument has been used widely by practitioners and can be administered quickly. The tool assesses for the following: 1) is the individual's mental change characteristic of an acute onset and fluctuating course, 2) inattention looks for signs of having difficulty focusing attention, 3) disorganized thinking such as incoherent thoughts and rambling, and 4) an altered level of consciousness such as alertness, vigilance, drowsiness, lethargic, stupor, or coma. The diagnosis of delirium by CAM requires the presence of features 1 and 2 and either 3 or 4 (RNAO, 2003)

Chemical Restraint: Any medication used for the purpose of restricting a patient's movement that is not related to the patient's medical or psychiatric treatment (Martin, 2002)

Delirium: Is an acute mental change from a person's baseline mental functioning. It is characterized by rapid onset of confusion, and is fluctuating in intensity. It may be caused by a number of underlying conditions including: medications, infections, and other disease processes 
that occur outside of the brain. Prompt treatment of underlying processes is required to regain baseline mental status. While people with dementia may also have delirium, people with delirium do not always have dementia. That is, delirium may be superimposed on dementia in hospitalized individual’s (Richards, 2011, Shapiro \& Mervis, 2007).

Dementia: Is an umbrella term pertaining to a variety of age-related disorders that affect the brain. The most common type of dementia is Alzheimer's disease (AD), also known as Alzheimer’s dementia (Gililland, 2007). Alzheimer’s disease is characterized by irreversible degenerative progressive changes in the brain as a result of changes in the function of two proteins, beta-amyloid and tau, which are part of plaques and neurofibrillary tangles that form in place of healthy brain tissue resulting in progressively worsening capabilities related to basic skills, speech, thought process and movement (Gililland, 2007).

HELP: A model of care to prevent delirium and functional decline in hospitalized older patients. A team-based, multidisciplinary approach to patient-centred care for older adults underpins this model. An Elder Life Specialists, Elder Life Nurse Specialists, program assistants and trained volunteers work together to identify at risk older adults and target interventions at reducing cognitive and functional decline (Inouye et at., 2000)..

Person-Centred Care: A philosophy based on the principles of trust, understanding, respect and individualism that influences and guides the practice of nursing and other disciplines (McCormack \& McCance, 2010). In the context of dementia care, Kitwood (1997) espouses the principles of person-centred care, including empowering the individual with dementia to exercise choice, to express their feelings, to use their abilities and to develop and maintain relationships, regardless of cognitive status. The Alzheimer's Society of Canada (2012) developed a personcentred care framework that advocates for people with dementia as individuals, whose care 
should be tailored to their unique needs based on their habits and desires. The framework ensures that people with dementia have an equal right to dignity and respect, irrespective of their cognitive functioning. Person-centred care means getting to know the person first, then understanding how their condition affects them.

P.I.E.C.E.S: Is a best practice learning initiative that provides a tool for those caring for person's with dementia to understand the cognitive and mental health needs of person's with dementia. The first three letters P-I-E, represent an individual's Physical, Intellectual and Emotional health. The $\mathrm{C}$ is the focus of care, for instance, maximizing the person's Capabilities to promote the highest quality of life. The E-S represents the Environment that an individual interacts with (physical and emotional) and the person's Social self (cultural, spiritual, life-story and support network) (MAREP, 2005)

Physical Restraint: Any physical device, material, or equipment attached or adjacent to the patient's body that he or she cannot remove. A physical restraint is used to restrict the patient's movement or normal access to ones’ body (Martin, 2002).

PLST: Is a model/framework that helps in the creation of individualized plans of care for individuals with dementia. The model is based on an individual's stress threshold which is said to be lower in the event of concurrent dementia. The goal of this model is to reduce stressors for the patient with dementia to reduce the likelihood of negative reactions or dysfunctional behavioural responses. Various interventions are provided to help improve function in the person with dementia. For example, modifying the physical environment, giving simple instructions instead of taking over, promoting self-worth, identify early anxious behaviours such as toe tapping or anxious face, and listening and watching for verbal and non-verbal cues of distress (Hall et al., 1987). 
Responsive behaviour: In the dementia context, are behaviours in response to something negative, frustrating, or confusing that is triggered by something in the person's environment (Purandare \& Burns, 2000). Responsive behaviours are similar to those behaviours outlined in the behavioural and psychological symptoms of dementia. However, responsive behaviors are conceptualized as meaningful and within a broader social and environmental context as opposed to pathological (Dupuis, Wiersma, \& Loiselle, 2012). 